Pacific Journal of Mathematic 


\title{
SUCCINCT AND REPRESENTATIONAL WITT RINGS
}

\author{
Jerrold L. Kleinstein ANd Alex Rosenberg
}

Dedicated to Gerhard Hochschild on the occasion of his 65th birthday

Three special classes of abstract Witt rings are studied. The classical description of the annihilator of a round form is generalized as is the description of the torsion subgroup of the Witt ring of a field. We translate some results of a previous paper into this abstract setting and also study Pfister forms there. We show how our special classes of abstract Witt rings relate to the Witt ring of classes of nondegenerate symmetric bilinear forms over a semilocal ring.

o. Introduction. This paper introduces various hypotheses on Witt rings for an elementary 2-group $G$, [14, Def. 3.12], that enable us to prove abstract counterparts of results known for Witt rings of bilinear forms over fields, often with very similar proofs. We consider three special classes of abstract Witt rings: "succinct" (Definition 2.10), "representational" (Definition 2.2), and "strongly representational" (Definition 4.1), each class being included in the one listed after it. The notion of representational was suggested by a similar definition in $[17,18]$ for reduced Witt rings for $G$. Our results are, of course, applicable to the Witt ring of a semilocal ring with mild restrictions on this semilocal ring.

Section 1 introduces the basic definitions and notations we need; these are carried over from [8]. In the second section we introduce the succinct and representational properties and investigate how they carry over to residue class Witt rings. We also show that in a representational Witt ring the description of the annihilator of a round element and in a succinct Witt ring the description of the torsion subgroup, are the usual ones. Several other known results are generalized, including a condition for a character of $G$ to induce a ring homomorphism from a Witt ring for $G$ to $Z$. The section ends by showing that a reduced representational Witt ring comes from a space of orderings in the sense of [17].

In $\S 3$ we generalize some of the results of [8] and [20] to Witt rings for $G$. In particular we give necessary and sufficient conditions for the existence of certain types of abelian group homomorphisms (semisignatures) from a Witt ring for $G$ to $Z$, and then use these homomorphisms to determine when an element of the Witt ring is weakly isotropic in an abstract sense. The section ends by adapting the proof in [20] for the equivalence of WAP and SAP, [7, Def. 1.5], to the case of a representational Witt ring for $G$. 
In $\S 4$ we introduce strongly representational Witt rings for $G$ and generalize several of the results concerning Pfister forms to be found in $[6,7]$ to this setting. We show that Pfister forms still are round and generalize the classical result that an isotropic Pfister form is hyperbolic to the abstract situation.

In the fifth section we show that some of the results of [3, $\S 5]$ carry over to representational Witt rings. We generalize [3, Prop. 5.1 , Thm. 5.3 (b), (c)] to certain classes of Witt rings for $G$. We further point out that by virtue of the main results of [17], Theorem 5.3(a) of [3] also carries over to representational reduced Witt rings for a finite group $G$.

In $\S 6$ we consider the Witt ring $W(C)$ of classes of nondegenerate symmetric bilinear $C$-forms where $C$ is a connected semilocal ring. In order to apply the previous results, we show that $W(C)$ is succinct if all the residue class fields of $C$ have at least 3 elements, and that $W(C)$ is strongly representational if 2 is a unit in $C$ or $C$ is a field of characteristic 2. By means of an example, we show that $W(C)$ need not be representational if $C$ is local with 2 not a unit in $C$. We also give another proof of the description of the annihilator of a round quadratic $C$-space of rank $\geqq 2$ due to Knebusch in [11], in case all the residue class fields of $C$ contain at least 3 elements.

We conclude this paper with a discussion in $\S 7$ of the derivation of some of the results in [8] and [20] from those in earlier section.

1. Notations. In this section we collect the definitions and notations we need from [8]. For a group $G$ of exponent 2, a ring $R=Z[G] / K$ is called a Witt ring for $G$ if $R_{t}$, the torsion subgroup of $R$, is 2-primary [14, Def. 3.12]. For $g$ in $G$ we denote the image of $g$ in $R$ by $\bar{g}$ although we shall often write 1 for the identity element of both $G$ and $R$. Every element of $R$ may be written as $\sum\left( \pm \bar{g}_{i}\right)$ for not necessarily distinct elements $g_{i}$ of $G$. We denote the multiplicative subgroup of $Z[G]$ consisting of the elements $\pm g$, $g$ in $G$, by $G^{\prime}$ and write $\bar{g}^{\prime}$ for $\pm \bar{g}$.

Definition 1.1. For $r$ in $R$, $\operatorname{dim}_{R} r$, or $\operatorname{dim} r$ if there is no possibility of confusion, is the smallest number $n$ such that $r=$ $\sum_{i=1}^{n} \bar{g}_{i}^{\prime}, g_{i}^{\prime}$ in $G^{\prime}$. Clearly for $r_{1}, \cdots, r_{m}$ in $R$, we always have $\operatorname{dim}\left(\sum_{1}^{m} r_{j}\right) \leqq \sum_{1}^{m} \operatorname{dim} r_{j}$.

Definition 1.2. For $g^{\prime}$ in $G^{\prime}$, the element $r$ in $R$ is said to represent the element $g^{\prime}$ of $G^{\prime}$, if there is an element $p$ in $R$ with $r=\bar{g}^{\prime}+p$ and $\operatorname{dim} p<\operatorname{dim} r$. The subset of $Z[G]$ represented by $r$ will be denoted by $D_{R}(r)=D(r)$. 
For the relation of these concepts with the presentation of $R$, cf. Remark 6.14.

Definition 1.3. For $g_{1}^{\prime}, \cdots, g_{n}^{\prime}$ in $G^{\prime}$, the element $\sum_{1}^{n} g_{i}^{\prime}$ of $Z[G]$ is said to be anisotropic for $R$ if $\operatorname{dim}\left(\sum_{1}^{n} \bar{g}_{i}^{\prime}\right)=n$. Otherwise $\sum_{1}^{n} \bar{g}_{i}^{\prime}$ will be called isotropic for $R$.

Lemma 1.4. (cf. [8, Lem. 1.4]). For $r$ in $R$, let $\sum_{1}^{n} g_{i}^{\prime}$ be an anisotropic representative of $r$ in $Z[G]$. Then $\sum_{1}^{n} g_{i}^{\prime}+g^{\prime}$, for some $g^{\prime}$ in $G^{\prime}$, is an element of $Z[G]$ isotropic for $R$ if and only if $-g^{\prime}$ is in $D\left(r^{\circ}\right)$.

Definition 1.5. If $R$ is a Witt ring for $G$, the set of ring homomorphisms $R \rightarrow Z$ is denoted by $X(R)$ and called the set of signatures of $R$.

REMARK 1.6. For $\sigma$ in $X(R)$, the ideal $\operatorname{ker} \sigma$ is a minimal nonmaximal prime ideal of $R$ and the mapping $\sigma \rightarrow \operatorname{ker} \sigma$ is a bijection of $X(R)$ onto the set of minimal non-maximal prime ideals of $R$ [14, Lem. 3.1 and Rem. 3.2]. Of course, by passing to inverse images in $Z[G]$, the set $X(R)$ is also bijective with the set of minimal nonmaximal prime ideals of $Z[G]$ containing $K$. By [14, Prop. 3.4], $X(R) \neq \varnothing$ if and only if $R_{t}=$ Nil $R$, the nilradical of $R$. Thus a Witt ring for $G$, with $X(R) \neq \varnothing$, is reduced if and only if it is torsion free, and in such a ring $x=y$ if and only if $\sigma(x)=\sigma(y)$ for all $\sigma$ in $X(R)$. Finally, since for all $g^{\prime}$ in $G^{\prime}$, we have $g^{\prime 2}=1$, we must have $\sigma\left(\bar{g}^{\prime}\right)= \pm 1$ for all $\sigma$ in $X(R)$ so that $X(R)$ may be identified with a subset of the character group $\hat{G}^{\prime}$ of $G^{\prime}$.

Definition 1.7. (cf. $[15, \S 4]$ ). (i) For any subset $M$ of $G^{\prime}$ in $Z[G]$, we set $V(M)=\left\{\sigma\right.$ in $X(R) \mid \sigma\left(\bar{g}^{\prime}\right)=1$ for all $g^{\prime}$ in $\left.M\right\}$.

(ii) For $Y \subset X(R)$, we put $\Gamma(Y)=\left\{g^{\prime}\right.$ in $G^{\prime} \mid \sigma\left(\bar{g}^{\prime}\right)=1$ for all $\sigma$ in $Y$.

(iii) A subset $Y$ of $X(R)$ is saturated if $Y=V(\Gamma(Y))$.

(iv) For $Y \subset X(R)$, we set $I(Y)=\bigcap_{\sigma \mathrm{in} Y} \operatorname{ker} \sigma$, an ideal of $R$.

(v) For any subset $M$ of $G^{\prime}$ in $Z[G]$ we denote the (proper) ideal of $R$ generated by $1-\bar{g}^{\prime}, g^{\prime}$ in $M$, by $a(M)$.

Definition 1.8. An additive homomorphism $\tau: R \rightarrow Z$ is called a semisignature if for all $g^{\prime}$ in $G^{\prime}$ we have $\tau\left(\bar{g}^{\prime}\right)= \pm 1$.

2. Representational and succinct Witt rings for $G$.

Lemma 2.1. (i ) For $g^{\prime}$ in $G^{\prime}$ and $r$ in $R$ we have $g^{\prime} D_{R}(r)=$ 
$D_{l i}\left(\bar{g}^{\prime} \cdot\right)$

(ii) For $g^{\prime}$ in $G^{\prime}$ we have $D\left(\bar{g}^{\prime}\right) \subset D\left(1+\bar{g}^{\prime}\right)$ if $1+\bar{g}^{\prime} \neq 0$ in $R$.

Proof. (i) According to Definition 1.2 an element $h_{1}^{\prime}$ of $G^{\prime}$ lies in $D(r)$ if and only if there exists an element $p_{1}$ of $R$ with $\operatorname{dim} p_{1}<\operatorname{dim} r$ such that $\bar{h}_{1}^{\prime}+p_{1}=r$, and an element $h_{2}^{\prime}$ of $G^{\prime}$ lies in $D\left(\bar{g}^{\prime} r\right)$ if and only if there exists an element $p_{2}$ of $R$ with $\operatorname{dim} p_{2}<$ $\operatorname{dim} \bar{g}^{\prime} r$ such that $\bar{h}_{2}^{\prime}+p_{2}=\bar{g}^{\prime} r$. Now from Definition 1.1 it is clear that for all $q$ in $R$ we have $\operatorname{dim}\left(\bar{g}^{\prime} q\right)=\operatorname{dim} q$, so that $g^{\prime} h_{1}^{\prime}$ lies in $D\left(\bar{g}^{\prime} r\right)$ and $g^{\prime} h_{2}$ lies in $D(r)$. Since $h_{2}^{\prime}=g^{\prime}\left(g^{\prime} h_{2}^{\prime}\right)$ this proves (i).

(ii) By [8, Rem. 1.24, Lem.], $\operatorname{dim}\left(1+\bar{g}^{\prime}\right)=2$ since $1+\bar{g}^{\prime} \neq 0$, and so $1+g^{\prime}$ is anisotropic for $R$. Now if $h^{\prime}$ lies in $D\left(\bar{g}^{\prime}\right)$, then $\bar{h}^{\prime}=\bar{g}^{\prime}$. Thus $1+\bar{h}^{\prime}=1+\bar{g}^{\prime}$ and since $\operatorname{dim} 1=1, \bar{h}^{\prime}$ is in $D(1+\bar{g})$ according to Definition 1.2.

Definition 2.2. (cf. $\left[17, O_{4}\right]$ ). A Witt ring $R$ for $G$ is called representational if for $r_{1} \neq 0, r_{2} \neq 0$ in $R$ with $\operatorname{dim}\left(r_{1}+r_{2}\right)=\operatorname{dim} r_{1}+$ $\operatorname{dim} r_{2}$ and $g^{\prime}$ in $D\left(r_{1}+r_{2}\right)$, there exist $g_{j}^{\prime}$ in $D\left(r_{j}\right), j=1,2$, with $g^{\prime}$ in $D\left(\bar{g}_{1}^{\prime}+\bar{g}_{2}^{\prime}\right)$. For the relation of this definition with the presentation of $R$, cf. Remark 6.14.

LEMMA 2.3. If $R$ is a Witt ring for $G$ and $\bar{g}_{j}^{\prime}$, and $r_{j}, j=1,2$ are as in Definition 2.2, then $g_{1}^{\prime}+g_{2}^{\prime}$ is anisotropic for $R$; in particular, $\bar{g}_{1}^{\prime}+\bar{g}_{2}^{\prime} \neq 0$ in $R$.

Proof. Since $g_{j}^{\prime}$ lies in $D\left(r_{j}\right), j=1,2$, by Definition 1.2 we have $\bar{g}_{j}^{\prime}+p_{j}=r_{j}$, and $\operatorname{dim} p_{j}<\operatorname{dim} r_{j}$. Thus $r_{1}+r_{2}=\left(\bar{g}_{1}^{\prime}+\bar{g}_{2}^{\prime}\right)+p_{1}+p_{2}$. Now if $g_{1}^{\prime}+g_{2}^{\prime}$ were isotropic for $R$, then by [8, Rem. 1.24, Lem.], $\bar{g}_{1}^{\prime}+\bar{g}_{2}^{\prime}=0$. Hence $\operatorname{dim}\left(r_{1}+r_{2}\right)=\operatorname{dim}\left(p_{1}+p_{2}\right)<\operatorname{dim} r_{1}+\operatorname{dim} r_{2}$. This contradicts the hypothesis on $r_{1}+r_{2}$ and so $g_{1}^{\prime}+g_{2}^{\prime}$ is anisotropic for $R$.

Proposition 2.4. Let $R$ be a Witt ring for $G$ and $r_{i}, i=1,2$, two elements of $R$. Then $R$ is representational if and only if whenever $\operatorname{dim}\left(r_{1}+r_{2}\right)<\operatorname{dim} r_{1}+\operatorname{dim} r_{2}$, there exists an element $g^{\prime}$ of $G^{\prime}$ with $g^{\prime}$ in $D\left(r_{1}\right)$ and $-g^{\prime}$ in $D\left(r_{2}\right)$.

Proof. Suppose $R$ is a representational Witt ring and let $r_{i}=\sum_{j=1}^{n_{i}} \bar{g}_{j i}^{\prime}, i=1,2$, with $n_{i}=\operatorname{dim} r_{i}$. Since $\sum_{j=1}^{n_{1}} g_{j 1}^{\prime}+\sum_{j=1}^{n_{2}} g_{j 2}^{\prime}$ is isotropic for $R$, but $\sum_{j=1}^{n_{1}} g_{j 1}^{\prime}$ is anisotropic for $R$, there exists a natural number $k$, with $k<n_{2}$, such that $\sum_{j=1}^{n_{1}} g_{j 1}^{\prime}+\sum_{j=1}^{k} g_{j 2}^{\prime}$ is anisotropic for $R$ but $\sum_{j=1}^{n_{1}} g_{j 1}^{\prime}+\sum_{j=1}^{k+1} g_{j 2}^{\prime}$ is isotropic for $R$. By Lemma 1.4 then $-g_{k+1,2}^{\prime}$ lies in $D\left(\sum_{j=1}^{n_{1}} \bar{g}_{j 1}^{\prime}+\sum_{j=1}^{k} \bar{g}_{j 2}^{\prime}\right)$. Since $R$ is representational there exist $g_{1}^{\prime}$ in $D\left(\sum_{j=1}^{n_{1}} \bar{g}_{j 1}^{\prime}\right)$ and $g_{2}^{\prime}$ in $D\left(\sum_{j=1}^{k} \bar{g}_{j 2}^{\prime}\right)$ such that 
$-g_{k+1,2}^{\prime}$ lies in $D\left(\bar{g}_{1}^{\prime}+\bar{g}_{2}^{\prime}\right)$. Hence for some element $h^{\prime}$ of $G^{\prime}$ we have $-g_{k+1,2}^{\prime}+\bar{h}^{\prime}=\bar{g}_{1}^{\prime}+\bar{g}_{2}^{\prime}$ or $\bar{h}^{\prime}=\bar{g}_{1}^{\prime}+\bar{g}_{2}^{\prime}+\bar{g}_{k+1,2}^{\prime}$ so that $g_{1}^{\prime}+g_{2}^{\prime}+g_{k+1,2}^{\prime}$ is isotropic for $R$. Furthermore, there exists an element $p$ in $R$ with $\bar{g}_{2}^{\prime}+p=\sum_{j=1}^{k} \bar{g}_{j 2}^{\prime}$ and $\operatorname{dim} p<k$. Therefore $\bar{g}_{2}^{\prime}+\bar{g}_{k+1,2}^{\prime}+p=$ $\sum_{j=1}^{k+1} \bar{g}_{j 2}^{\prime}$ and so if $g_{2}^{\prime}+g_{k+1,2}^{\prime}$ were isotropic for $R$, so would $\sum_{j=1}^{k+1} g_{j 2}^{\prime}$ be, contradicting $\operatorname{dim} r_{2}=n_{2}$. Since $g_{2}^{\prime}+g_{k+1,2}^{\prime}$ is anisotropic for $R$, but $g_{1}^{\prime}+g_{2}^{\prime}+g_{k+1,2}^{\prime}$ is isotropic for $R$, Lemma 1.4 shows that $-g_{1}^{\prime}$ is in $D\left(\bar{g}_{2}^{\prime}+\bar{g}_{k+1,2}^{\prime}\right) \subset D\left(\sum_{1}^{k+1} \bar{g}_{j_{2}}^{\prime}\right) \subset D\left(r_{2}\right)$, which shows that the condition holds.

Now suppose that whenever $\operatorname{dim}\left(r_{1}+r_{2}\right)<\operatorname{dim} r_{1}+\operatorname{dim} r_{2}$, for any $r_{1}, r_{2}$ in $R$, there exists $g^{\prime}$ in $D\left(r_{1}\right)$ such that $-g^{\prime}$ is in $D\left(r_{2}\right)$. The proof that $R$ is then representational is based upon the proof of [18, Lem. 1.3]. Thus, let $r_{i}, i=1,2$ be elements of $R$ with $\operatorname{dim}\left(r_{1}+r_{2}\right)=\operatorname{dim} r_{1}+\operatorname{dim} r_{2}$, and let $g^{\prime}$ be in $D\left(r_{1}+r_{2}\right)$. By Lemma 1.4, $\operatorname{dim}\left(r_{1}+r_{2}-\bar{g}^{\prime}\right)<\operatorname{dim} r_{1}+\operatorname{dim} r_{2}+1$. Now $\operatorname{dim}\left(r_{2}-\bar{g}^{\prime}\right) \leqq$ $\operatorname{dim} r_{2}+1$. If $\operatorname{dim}\left(r_{2}-\bar{g}^{\prime}\right)<\operatorname{dim} r_{2}+1$ then by Lemma $1.4, g^{\prime}$ is in $D\left(r_{2}\right)$ and so $g^{\prime}$ is in $D\left(\bar{g}_{1}^{\prime}+\bar{g}^{\prime}\right)$ for any $g_{1}^{\prime}$ in $D\left(r_{1}\right)$. So suppose $\operatorname{dim}\left(r_{2}-\bar{g}^{\prime}\right)=$ $\operatorname{dim} r_{2}+1$, then there is a $g_{1}^{\prime}$ such that $g_{1}^{\prime}$ is in $D\left(r_{1}\right)$ and $-g_{1}^{\prime}$ is in $D\left(r_{2}-\bar{g}^{\prime}\right)$. Again by Lemma 1.4, this means $\operatorname{dim}\left(r_{2}-\bar{g}^{\prime}+\bar{g}_{1}^{\prime}\right)<$ $\operatorname{dim} r_{2}+2$. Now if $\bar{g}^{\prime}=\bar{g}_{1}^{\prime}$, then $g^{\prime}$ lies in $D\left(\bar{g}_{1}^{\prime}+\bar{g}_{2}^{\prime}\right)$ for any $g_{2}^{\prime}$ in $D\left(r_{2}\right)$. So assume $\operatorname{dim}\left(-\bar{g}^{\prime}+\bar{g}_{1}^{\prime}\right)=2$. Then there is a $g_{2}^{\prime}$ in $D\left(r_{2}\right)$ with $-g_{2}^{\prime}$ in $D\left(-\bar{g}^{\prime}+\bar{g}_{1}^{\prime}\right)$. Thus, there exists an $h^{\prime}$ in $G^{\prime}$ with $-\bar{g}_{2}^{\prime}+\bar{h}^{\prime}=$ $-\bar{g}^{\prime}+\bar{g}_{1}^{\prime}$ or $\bar{g}^{\prime}+\bar{h}^{\prime}=\bar{g}_{1}^{\prime}+\bar{g}_{2}^{\prime}$. By Lemma $2.3, g_{1}^{\prime}+g_{2}^{\prime}$ is anisotropic, which means $g^{\prime}$ lies in $D\left(\bar{g}_{1}^{\prime}+\bar{g}_{2}^{\prime}\right)$. Thus $R$ is representational.

Let $\chi$ be any character of $G$, i.e., a homomorphism from $G$ to $\{ \pm 1\}$. The mapping $\chi$ extends to a ring homomorphism $\boldsymbol{Z}[G] \rightarrow \boldsymbol{Z}$ via $\sum n_{g} g \rightarrow \sum n_{g} \chi(g)$ which we also denote by $\chi$.

Lemma 2.5. Let $R$ be a representational Witt ring for $G$ and $\chi$ a character of $G$ such that if $\chi\left(g^{\prime}\right)=1$ and $1+\bar{g}^{\prime} \neq 0$, then $\chi\left(D_{R}\left(1+\bar{g}^{\prime}\right)\right)=1$. Then if $1+\sum_{1}^{n} g_{i}^{\prime}$ is an element of $Z[G]$ anisotropic for $R$ and $\chi\left(g_{i}^{\prime}\right)=1, i=1, \cdots, n$, then $\chi\left(D\left(1+\sum_{1}^{n} \bar{g}_{i}^{\prime}\right)\right)=1$, also.

Proof. We shall use induction on $n$ and note that the lemma is true for $n=1$ by hypothesis since by [8, Rem. 1.24, Lem.] dim $\left(1+\bar{g}^{\prime}\right)=2$. Suppose Lemma 2.5 holds for $n-1$. Let $g^{\prime}$ lie in $D\left(1+\sum_{1}^{n-1} \bar{g}_{i}^{\prime}+\bar{g}_{n}^{\prime}\right)$. Since $1+\sum_{1}^{n} g_{i}^{\prime}$ is anisotropic for $R$ we clearly have $n+1=\operatorname{dim}\left(1+\sum_{1}^{n} \bar{g}_{i}^{\prime}\right)=\operatorname{dim}\left(1+\sum_{1}^{n-1} \bar{g}_{i}^{\prime}\right)+\operatorname{dim} \bar{g}_{n}^{\prime}$ and so, since $R$ is representational, there exist $h_{1}^{\prime}$ in $D\left(1+\sum_{1}^{n-1} \bar{g}_{i}^{\prime}\right)$ and $h_{2}^{\prime}$ in $D\left(\bar{g}_{n}^{\prime}\right)$ with $g^{\prime}$ in $D\left(\bar{h}_{1}^{\prime}+\bar{h}_{2}^{\prime}\right)$. Since $1+\sum_{1}^{n} g_{i}^{\prime}$ is anisotropic for $R$, it is clear that $1+\bar{g}_{n}^{\prime} \neq 0$ in $R$, and thus by Lemma 2.1(ii), $h_{2}^{\prime}$ lies in $D\left(1+\bar{g}_{n}^{\prime}\right)$. Hence the induction assumption and the hypothesis of 
Lemma 2.5 yield $\chi\left(h_{j}^{\prime}\right)=1, j=1,2$. But by Lemma 2.3 we have $1+\bar{h}_{1}^{\prime} \bar{h}_{2}^{\prime} \neq 0$ and so by hypothesis $\chi\left(D\left(1+\bar{h}_{1}^{\prime} \bar{h}_{2}^{\prime}\right)\right)=1$ since $\chi\left(h_{1}^{\prime} h_{2}^{\prime}\right)=1$. Finally, since $g^{\prime}$ lies in $D\left(\bar{h}_{1}^{\prime}+\bar{h}_{2}^{\prime}\right)=h_{1}^{\prime} D\left(1+\bar{h}_{1}^{\prime} \bar{h}_{2}^{\prime}\right)$ by Lemma 2.1(i), we obtain $\chi\left(g^{\prime}\right)=1$, proving Lemma 2.5.

THEOREM 2.6. Let $R=Z[G] / K$ be a representational Witt ring for $G$ with $X(R) \neq \varnothing$ and $\chi$ a character of $G$. If for all $g^{\prime}$ in $G^{\prime}$ with $1+\bar{g}^{\prime} \neq 0$ in $R$ and $\chi\left(g^{\prime}\right)=1$ we have $\chi\left(D\left(1+\bar{g}^{\prime}\right)\right)=1$, then $\chi(K)=0$, so that $\chi$ induce a signature of $R$.

Proof. Let $g_{1}^{\prime}, \cdots, g_{n}^{\prime}$ be elements of $G^{\prime}$ such that $\chi\left(g_{i}^{\prime}\right)=1$, $i=1, \cdots, n$, and suppose for all $\sigma$ in $X(R)$ we have $\sigma\left(\bar{g}_{i}^{\prime}\right)=-1$ for at least one $i=1, \cdots, n$. Let $P=\Pi_{1}^{n}\left(1+\bar{g}_{i}^{\prime}\right)=\sum_{1}^{2^{n}} \bar{d}_{i}^{\prime}$ with $d_{i}^{\prime}$ in $G^{\prime}$. Since $\sigma\left(1+\bar{g}_{i}^{\prime}\right)=0$ for at least one $\bar{g}_{i}^{\prime}$ and every $\sigma$ in $X(R)$, we have $\sigma(P)=0$ for each $\sigma$ in $X(R)$. By [14, Prop. 3.15], $P$ lies in $R_{t}$, so that there is a natural number $m$ with $m P=0$. Hence $\sum_{i=1}^{2^{n}} \sum_{1}^{m} d_{i}^{\prime}$ is isotropic for $R$. Since $d_{1}^{\prime}=1$ is anisotropic for $R$ there is a natural number $l$ so that $x=\sum_{i=1}^{l} \sum_{1}^{m} d_{i}^{\prime}$ (or $\sum_{1}^{l-1} \sum_{1}^{m} d_{l}^{\prime}+\sum_{1}^{l^{\prime}} d_{l}^{\prime}$, $l^{\prime}<m$ ) is anisotropic for $R$ but $x+d_{l+1}^{\prime}\left(\right.$ or $x+d_{l}^{\prime}$ ) is not. By Lemma 1.4 then, $-d_{l+1}^{\prime}$ (or $-d_{l}^{\prime}$ ) lies in $D(\bar{x})$, where $\bar{x}$ denotes the image of $x$ in $R$. By Lemma 2.5, therefore, $\chi\left(-d_{l+1}^{\prime}\right)$ (or $\chi\left(-d_{l}^{\prime}\right)$ ) is 1 , contradicting $\chi\left(d_{i}^{\prime}\right)=1, i=1, \cdots, 2^{n}$. Hence there exists a $\sigma$ in $X(R)$ with $\sigma\left(\bar{g}_{i}^{\prime}\right)=1, i=1, \cdots, n$. Now let $k$ denote an element of the ideal $K$ of $Z[G]$. We write $k=\sum g_{i}^{\prime}+\sum h_{i}^{\prime}$ with $\chi\left(g_{i}^{\prime}\right)=1$, $\chi\left(h_{i}^{\prime}\right)=-1$. But then there exists a signature $\sigma$ of $R$ with $\sigma\left(\bar{g}_{i}^{\prime}\right)=$ $\sigma\left(-\bar{h}_{i}^{\prime}\right)=1$ and since $\sigma$ is a ring homomorphism of $R$ to $Z$ we must have $0=\sum \sigma\left(\bar{g}_{i}^{\prime}\right)+\sum \sigma\left(\bar{h}_{i}^{\prime}\right)=\chi(k)$. Thus $\chi(K)=0$, and $\chi$ induces a signature on $R$.

REMARK (i ) Theorem 2.6 has already been proved in case $G$ is finite and $R_{t}=0$ in [17, Thm. 4.1].

(ii) Let $C$ be a connected semilocal ring and $W(C)$ the Witt ring of classes of symmetric nondegenerate bilinear $C$-forms. If no residue class field of $C$ contains 2 or 4 elements an analogue of Theorem 2.6 has been proved in [15, Prop. 2.4]. However our Theorem 2.6 only yields this result if 2 is a unit in $C$, since we shall show in Example 6.8 that if 2 fails to be a unit in $C$ then $W(C)$ may fail to be representational, but will prove in Proposition 6.7 that if 2 is a unit in $C$ then $W(C)$ is representational.

Proposition 2.7. Let $R$ be a representational Witt ring for $G$ and $r_{1}, \cdots, r_{n}$ nonzero elements of $R$ with $\operatorname{dim}\left(\sum_{1}^{n} r_{i}\right)=\sum_{1}^{n} \operatorname{dim} r_{i}$. If $g^{\prime}$ lies in $D\left(\sum_{1}^{n} r_{i}\right)$ there exist $g_{i}^{\prime}$ in $D\left(r_{i}\right), i=1, \cdots, n$, such that $g^{\prime}$ is in $D\left(\sum_{1}^{n} \bar{g}_{i}^{\prime}\right)$ and $\operatorname{dim} \sum_{1}^{n} \bar{g}_{i}^{\prime}=n$. 
Proof. For $n=2$ this is simply Definition 2.2 and Lemma 2.3. We use induction on $n>2$. Let $p=\sum_{2}^{n} r_{i}$, then clearly $\operatorname{dim} p=$ $\sum_{2}^{n} \operatorname{dim} r_{i}$ and so $\operatorname{dim}\left(r_{1}+p\right)=\operatorname{dim} r_{1}+\operatorname{dim} p$. By Definition 2.2 and Lemma 2.3, there then exist $g_{1}^{\prime}$ in $D\left(r_{1}\right)$ and $h^{\prime}$ in $D(p)$ with $g^{\prime}$ in $D\left(\bar{g}_{1}^{\prime}+\bar{h}^{\prime}\right)$ and $g_{1}^{\prime}+h^{\prime}$ anisotropic for $R$. This means that there exists an element $y^{\prime}$ in $G^{\prime}$ with $\bar{g}^{\prime}+\bar{y}^{\prime}=\bar{g}_{1}^{\prime}+\bar{h}^{\prime}$ since $\bar{g}^{\prime}=\bar{g}_{1}^{\prime}+\bar{h}^{\prime}$ would mean that $g_{1}^{\prime}+h^{\prime}$ is isotropic for $R$, contradicting Lemma 2.3. Now by the induction assumption, there exists $g_{i}^{\prime}$ in $D\left(r_{i}\right), i=2, \cdots, n$, with $h^{\prime}$ in $D\left(\sum_{2}^{n} \bar{g}_{i}^{\prime}\right)$ and $\sum_{2}^{n} g_{i}^{\prime}$ anisotropic for $R$. Thus there is an element $q$ in $R$ with $\operatorname{dim} q \leqq n-2$ and $\bar{h}^{\prime}+q=\sum_{2}^{n} \bar{g}_{i}^{\prime}$. Hence $\bar{g}_{1}^{\prime}+\bar{h}^{\prime}+q=\bar{g}^{\prime}+\bar{y}^{\prime}+q=\sum_{1}^{n} \bar{g}_{i}^{\prime}$. Since $g_{i}^{\prime}$ lies in $D\left(r_{i}\right)$, we have for $i=1, \cdots, n, \bar{g}_{i}^{\prime}+q_{i}=r_{i}$ with $\operatorname{dim} q_{i} \leqq \operatorname{dim} r_{i}-1$. Now $\sum_{1}^{n} \operatorname{dim} r_{i}=\operatorname{dim} \sum_{1}^{n} r_{i} \leqq \operatorname{dim} \sum_{1}^{n} \bar{g}_{i}^{\prime}+\sum_{1}^{n} \operatorname{dim} r_{i}-n$, which clearly forces $\operatorname{dim} \sum_{1}^{n} \bar{g}_{i}^{\prime}=n$. Thus $\sum_{1}^{n} g_{i}^{\prime}$ is anisotropic for $R$ and $\operatorname{dim}\left(\bar{y}^{\prime}+q\right) \leqq$ $1+n-2=n-1<\operatorname{dim} \sum_{1}^{n} \bar{g}_{i}^{\prime}$. Hence $g^{\prime}$ lies in $D\left(\sum_{1}^{n} \bar{g}_{i}^{\prime}\right)$, proving Proposition 2.7.

Lemma 2.8. Let $R$ be a representational Witt ring for $G$ and $\sum_{i=1}^{n} \sum_{j=1}^{m_{i}} g_{i}^{\prime} h_{i j}^{\prime}$ be an element of $Z[G]$ which is isotropic for $R$ but with $p_{i}=\sum_{j=1}^{m_{i}} h_{i j}^{\prime}$ anisotropic for $R$. Then there exist $t_{i}^{\prime}$ in $D\left(\bar{p}_{i}\right)$ with $\sum_{1}^{n} g_{i}^{\prime} t_{i}^{\prime}$ isotropic for $R$.

Proof. The element $g_{1}^{\prime} h_{11}^{\prime}$ is anisotropic for $R$ whereas the element $\sum_{i=1}^{n} \sum_{j=1}^{m_{i}} g_{i}^{\prime} h_{i j}^{\prime}$ of $Z[G]$ is isotropic for $R$. Thus summing in the usual order there is a first place where the sum becomes isotropic for $R$, at $g_{k+1}^{\prime} h_{k+1, l}^{\prime}$, say. This means that $z_{1}=\sum_{1}^{k} g_{i}^{\prime} p_{i}+$ $\sum_{j=1}^{l-1} g_{k+1}^{\prime} h_{k+1, j}^{\prime}$ is anisotropic for $R$ but $z_{1}+g_{k+1}^{\prime} h_{k+1, l}^{\prime}$ is isotropic for $R$. (If $l=1$, the empty sum $\sum_{j=1}^{l-1} g_{k+1}^{\prime} h_{k+1, j}^{\prime}$ is taken to be 0.) By Lemma 1.4, $-g_{k+1}^{\prime} h_{k+1, l}^{\prime}$ is in $D\left(\bar{z}_{1}\right)$, where $\bar{z}_{1}$ denotes the image of $z_{1}$ in $R$. Since $z_{1}$ is anisotropic for $R$ we have $\operatorname{dim} \bar{z}_{1}=\sum_{1}^{k} \operatorname{dim} \bar{p}_{i}+l-1$ and Proposition 2.7 is applicable, yielding elements $x_{i}^{\prime}, y_{j}^{\prime}$ in $G^{\prime}$, $i=1, \cdots, k, j=1, \cdots, l-1$, with $x_{i}^{\prime}$ in $D\left(\bar{g}_{i}^{\prime} \bar{p}_{i}\right), y_{j}^{\prime}$ in $D\left(\bar{g}_{k+1}^{\prime} \bar{h}_{k+1, j}^{\prime}\right)$, $-g_{k+1}^{\prime} h_{k+1, l}^{\prime}$ in $D\left(\sum_{i=1}^{k} \bar{x}_{i}^{\prime}+\sum_{j=1}^{l-1} \bar{y}_{j}^{\prime}\right)$ and $\sum_{i=1}^{k} x_{i}^{\prime}+\sum_{j=1}^{l-1} y_{j}^{\prime}$ anisotropic for $R$. By Lemma 2.1(i), $t_{i}^{\prime}=g_{i}^{\prime} x_{i}^{\prime}$ lies in $D\left(\bar{p}_{i}\right)$ and $x_{i}^{\prime}=g_{i}^{\prime} t_{i}^{\prime}$. Moreover, since $y_{j}^{\prime}$ is in $D\left(\bar{g}_{k+1}^{\prime} \bar{h}_{k+1, j}^{\prime}\right)$ we must have $\bar{y}_{j}^{\prime}=\bar{g}_{k+1}^{\prime} \bar{h}_{k+1, j}^{\prime}$. Hence $-g_{k+1}^{\prime} h_{k+1, l}^{\prime}$ lies in $D\left(\bar{z}_{2}\right)$ where $z_{2}=\sum_{i=1}^{k} g_{i}^{\prime} t_{i}^{\prime}+\sum_{j=1}^{l-1} g_{k+1}^{\prime} h_{k+1, j}^{\prime}$, and $\operatorname{dim} \bar{z}_{2}=k+l-1$. Thus once more by Lemma 1.4, the element $z_{2}+g_{k+1}^{\prime} h_{k+1, l}^{\prime}$ of $Z[G]$ is isotropic for $R$. Hence if $l=1$ we are done with $t_{k+1}^{\prime}=h_{k+1,1}^{\prime}$ and, for $i>k+1$, the element $t_{i}^{\prime}$ an arbitrary element of $D\left(\bar{p}_{i}\right)$.

If $l>1$, then since $\sum_{j=1}^{m_{k+1}} h_{k+1, j}^{\prime}=p_{k+1}$ is anisotropic for $R$ so is $\sum_{j=1}^{l} g_{k+1}^{\prime} h_{k+1, j}^{\prime}$. On the other hand, $z_{2}+g_{k+1}^{\prime} h_{k+1, l}^{\prime}$ is isotropic, hence there exists a natural number $s \leqq k-1$ with $z_{3}=\sum_{j=1}^{l} g_{k+1}^{\prime} h_{k+1, j}^{\prime}+$ $\sum_{1}^{s} g_{i}^{\prime} t_{i}^{\prime}$ anisotropic for $R$, but $z_{3}+g_{s+1}^{\prime} t_{s+1}^{\prime}$ isotropic for $R$. Again by 
Lemma 1.4, this means that $-g_{s+1}^{\prime} t_{s+1}^{\prime}$ lies in $D\left(\bar{z}_{3}\right)$. Now clearly $\operatorname{dim} \bar{z}_{3}=l+s$ so that we may apply Proposition 2.7 again to obtain elements $u_{k+1}^{\prime}, v_{i}^{\prime}, i=1, \cdots, s$ in $G^{\prime}$ with $-g_{s+1}^{\prime} t_{s+1}^{\prime}$ in $D\left(\bar{u}_{k+1}^{\prime}+\sum_{1}^{s} \bar{v}_{i}^{\prime}\right)$, $u_{k+1}^{\prime}$ in $D\left(\sum_{j=1}^{l} \bar{g}_{k+1}^{\prime} \bar{h}_{k+1, j}^{\prime}\right)$, and $v_{i}^{\prime}$ in $D\left(\bar{g}_{i}^{\prime} \bar{t}_{i}^{\prime}\right)$. Then $\bar{v}_{i}^{\prime}=\bar{g}_{i}^{\prime} \bar{t}_{i}^{\prime}$ and by Lemma 2.1(i), $u_{k+1}^{\prime}=g_{k+1}^{\prime} t_{k+1}^{\prime}$ with $t_{k+1}^{\prime}$ in $D\left(\sum_{j=1}^{l} \bar{h}_{k+1, j}^{\prime}\right) \subset D\left(\bar{p}_{k+1}\right)$. Hence $-g_{s+1}^{\prime} t_{s+1}^{\prime}$ lies in $D\left(\bar{g}_{k+1}^{\prime} \bar{t}_{k+1}^{\prime}+\sum_{1}^{s} \bar{g}_{i}^{\prime} \bar{t}_{i}^{\prime}\right)$, which by a final application of Lemma 1.4 shows that $\sum_{1}^{n} g_{i}^{\prime} t_{i}^{\prime}$, with $t_{i}$ in $D\left(\bar{p}_{i}\right)$, is isotropic for $R$, where for $s+1<i \leqq k$ and $i>k+1, t_{i}$ is an arbitrary element of $D\left(\bar{p}_{i}\right)$.

Lemma 2.9. Let $R$ be a Witt ring for $G$ and $r$ an element of $R$. If for a semisignature $\tau$ of $R$ (Definition 1.8) we have $\tau(r)=$ $\operatorname{dim} r$, then $\tau\left(\bar{g}^{\prime}\right)=1$ for all $g^{\prime}$ in $D(r)$.

Proof. Since $g^{\prime}$ is in $D(r)$ there exists an element $q$ in $R$ with $\operatorname{dim} q<\operatorname{dim} r$ and $\bar{g}^{\prime}+q=r$. Thus we have $\tau\left(\bar{g}^{\prime}\right)=\tau(r)-\tau(q)$ for any semisignature $\tau$. Since $|\tau(q)| \leqq \operatorname{dim} q$ always, if $\tau(r)=\operatorname{dim} r$, then $\tau\left(\bar{g}^{\prime}\right)>0$ and so $\tau\left(\bar{g}^{\prime}\right)=1$.

Definition 2.10. A Witt ring $R$ for a group $G$ is called succinct if for any nonempty saturated set of signatures $Y$ of $R$, elements $g_{i}^{\prime}, i=1, \cdots, n$, of $G^{\prime}$, and elements $t_{i j}^{\prime}$ of $\Gamma(Y), j=1, \cdots, m_{i}$, such that $\sum_{i=1}^{n} \sum_{j=1}^{m_{i}} g_{i}^{\prime} t_{i j}^{\prime}$ is isotropic for $R$, there exist $t_{i}^{\prime}$ in $\Gamma(Y)$, $i=1, \cdots, n$, such that $\sum_{1}^{n} g_{i}^{\prime} t_{i}^{\prime}$ is isotropic for $R$.

THEOREM 2.11. A representational Witt ring for $G$ is succinct.

REMARK. We shall see in Remark 6.9 (i) that the inclusions $\{$ Witt rings for $G\} \supset\{$ succinct Witt rings for $G\} \supset\{$ representational Witt rings for $G\}$ are all proper.

Proof of Theorem 2.11. Note that since for all $\sigma$ in $Y$, we have $\sigma\left(\bar{t}_{i j}^{\prime}\right)=1$ we have $\sigma\left(\sum_{j=1}^{m_{i}} \bar{t}_{i j}^{\prime}\right)=m_{i}$, for the $t_{i j}^{\prime}$ and $Y$ of Definition 2.10. Hence the element $\sum_{j=1}^{m_{i}} t_{i j}^{\prime}$ of $Z[G]$ is anisotropic for $R$. By Lemma 2.8 then, there exist elements $t_{i}^{\prime}$ in $D\left(\sum_{j=1}^{m_{i}} \bar{t}_{i j}^{\prime}\right)$ with $\sum_{1}^{n} g_{i}^{\prime} t_{i}^{\prime}$ isotropic for $R$. Furthermore, by Lemma 2.9, since for $\sigma$ in $Y$, we have $\sigma\left(\sum_{j=1}^{m_{i}} \bar{t}_{i j}^{\prime}\right)=\operatorname{dim}\left(\sum_{j=1}^{m_{i}} \bar{t}_{i j}^{\prime}\right)$, we must have $\sigma\left(\bar{t}_{i}^{\prime}\right)=1$, so that $t_{i}^{\prime}$ lies in $\Gamma(Y)$ and Theorem 2.11 is proved.

COROLLARY 2.12. Let $R$ be a representational Witt ring for $G, p=\sum_{1}^{n} \bar{g}_{i}^{\prime}, q=\sum_{1}^{m} \bar{h}_{j}^{\prime}$ elements of $R$ with $\operatorname{dim} p=n$ and $\operatorname{dim} q=m$. If $\operatorname{dim} p q<m n$ then there exist $t_{i}^{\prime}$ in $D(q)$ with $\sum_{1}^{n} g_{i}^{\prime} t_{i}^{\prime}$ isotropic for $R$. 
Proof. This is immediate from Lemma 2.8 with $h_{i j}^{\prime}=h_{j}^{\prime}$ and $m_{i}=m$.

Lemma 2.13. Let $R$ be a Witt ring for $G$ and $\varphi$ an element of an $R$-module. Let $T$ be the subgroup of $G^{\prime}$ consisting of all $g^{\prime}$ in $G^{\prime}$ with $\bar{g}^{\prime} \varphi=\varphi$ and denote the annihilator of $\varphi$ in $R$ by $\operatorname{Ann}_{R}(\varphi)$. If for every $p$ in $\operatorname{Ann}_{R}(\varphi), p=\sum_{1}^{n} \bar{g}_{i}^{\prime}, \operatorname{dim} p=n$, there exist $t_{i}^{\prime}$ in $T, i=1, \cdots, n$, with $\sum_{1}^{n} g_{i}^{\prime} t_{i}^{\prime}$ isotropic for $R$, then $\operatorname{Ann}_{R}(\varphi)=\mathfrak{a}(T)$, the ideal generated by $1-t$, with $t$ in $T$.

Proof. Clearly $a(T) \subset \operatorname{Ann}_{R}(\varphi)$. To prove the opposite inclusion we proceed by induction on $\operatorname{dim} p$. Since all elements of $R$ of dimension 1 are units, $\operatorname{Ann}_{R}(\varphi)$ only contains elements of dimension $\geqq 2$. Let $\left(\bar{g}_{1}^{\prime}+\bar{g}_{2}^{\prime}\right) \varphi=0$. Then $-g_{1}^{\prime} g_{2}^{\prime}$ is in $T$ and thus $\bar{g}_{1}^{\prime}+\bar{g}_{2}^{\prime}=$ $\bar{g}_{1}^{\prime}\left(1-\left(-\bar{g}_{1}^{\prime} \bar{g}_{2}^{\prime}\right)\right)$ is in $\alpha(T)$. If $p=\sum_{1}^{n} \bar{g}_{i}^{\prime}, \operatorname{dim} p=n$, is an element of $\operatorname{Ann}_{R}(\varphi)$, then by hypothesis there are elements $t_{i}^{\prime}, i=1, \cdots, n$, in $T$ with $\operatorname{dim}\left(\sum_{1}^{n} \bar{g}_{i}^{\prime} \bar{t}_{i}^{\prime}\right)<n$. But $p=\sum_{1}^{n} \bar{g}_{i}^{\prime}\left(1-\bar{t}_{i}^{\prime}\right)+\sum_{1}^{n} \bar{g}_{i}^{\prime} \bar{t}_{i}^{\prime} \equiv \sum_{1}^{n} \bar{g}_{i}^{\prime} \bar{t}_{i}^{\prime}$ $\bmod a(T)$. By the induction assumption, $\sum_{1}^{n} \bar{g}_{i}^{\prime} \bar{t}_{i}^{\prime}$ lies in $a(T)$ since it clearly is still in $\operatorname{Ann}_{R}(\varphi)$, so $p$ does also, proving $\operatorname{Ann}_{R}(\varphi) \subset a(T)$ and Lemma 2.13.

Definition 2.14. Let $R$ be a Witt ring for $G$. An element $r \neq 0$ of $R$ is called round if for all $g^{\prime}$ in $D(r)$, we have $\bar{g}^{\prime} r=r$.

THEOREM 2.15. Let $r$ be a round element for a representational Witt ring $R$ for $G$. Then $\operatorname{Ann}_{R}(r)=\mathfrak{a}(D(r))$.

Proof. By Definition 2.14, we have $D(r) \subset T=\left\{g^{\prime}\right.$ in $\left.G^{\prime} \mid \bar{g}^{\prime} r=r\right\}$ and by Corollary 2.12 and Lemma 2.13, then $\mathrm{Ann}_{R}(r)=\mathfrak{a}(T)$. Let $\sum_{1}^{n} g_{i}^{\prime}$ be an anisotropic representative of $r$. Clearly $g_{1}^{\prime}$ lies in $D(r)$ so that $r=\bar{g}_{1}^{\prime} r=1+\sum_{2}^{n} \bar{g}_{1}^{\prime} \bar{g}_{i}^{\prime}$. Hence for $t^{\prime}$ in $T$, we have $r=\bar{t}^{\prime} r=$ $\bar{t}^{\prime}+\sum_{2}^{n} \bar{t}^{\prime} \bar{g}_{1}^{\prime} \bar{g}_{i}^{\prime}$ so that $t^{\prime}$ lies in $D(r)$ according to Definition 1.2. Hence $D(r) \supset T$ so $D(r)=T$ and $\operatorname{Ann}_{R}(r)=\mathfrak{a}(D(r))$.

Proposition 2.16. (cf. [6, Thm. 1.4; 4, Satz 14, Kor. 2]). Let $R$ be a representational Witt ring for $G$ and let $r$ be a round element of $R$. If $0 \neq q$ lies in $R r$, there exists an element $p$ in $R$ with $q=p r$ and $\operatorname{dim} q=\operatorname{dim} p \operatorname{dim} r$. Further, for $g^{\prime}$ in $D(q)$ there exists an element $p$ in $R$ satisfying the above condition and with $g^{\prime}$ in $D(p)$.

Proof. Let $p$ be an element of $R$ of minimal dimension such that $q=p r$. Let $p=\sum_{1}^{n} \bar{g}_{i}^{\prime}$ with $n=\operatorname{dim} p$ and set $\operatorname{dim} r=m$. If $\operatorname{dim} q<m n$, then by Corollary 2.12, there exist $t_{i}^{\prime}$ in $D(r)$ with 
$\sum_{1}^{n} g_{i}^{\prime} t_{i}^{\prime}$ isotropic for $R$. Since $r$ is round and $t_{i}^{\prime}$ lies in $D(r)$ we have $\bar{t}_{i}^{\prime} r=r$ so that $q=p r=\left(\sum_{1}^{n} \bar{g}_{i}^{\prime} \bar{t}_{i}^{\prime}\right) r$ and since $\operatorname{dim}\left(\sum_{1}^{n} \bar{g}_{i}^{\prime} \bar{t}_{i}^{\prime}\right)<n$, this contradicts the choice of $p$ so that $\operatorname{dim} q=m n$.

Now if $g^{\prime}$ is in $D(q)=D\left(\sum_{1}^{n} \bar{g}_{i}^{\prime} r\right)$, Proposition 2.7 proves the existence of $h_{i}^{\prime}$ in $D\left(\bar{g}_{i}^{\prime} r\right)$ such that $g^{\prime}$ lies in $D\left(\sum_{1}^{n} \bar{h}_{i}^{\prime}\right)$ and $\operatorname{dim}\left(\sum_{1}^{n} \bar{h}_{i}^{\prime}\right)=n$. Now by Lemma 2.1(i) we have $D\left(\bar{g}_{i}^{\prime} r\right)=g_{i}^{\prime} D(r)$; hence there are $t_{i}^{\prime}$ in $D(r)$ with $h_{i}^{\prime}=g_{i}^{\prime} t_{i}^{\prime}$. But then $\left(\sum_{1}^{n} \bar{h}_{i}^{\prime}\right) r=p r=q$ and $\sum_{1}^{n} \bar{h}_{i}^{\prime}$ is the required element.

Definition 2.17. An $m$-fold Pfister element in a Witt ring $R$ for $G$ is any element of the form $\prod_{1}^{m}\left(1+\bar{g}_{i}^{\prime}\right)=\sum_{1}^{2^{m}} \bar{d}_{i}^{\prime}$.

LEMMA 2.18. Let $R$ be a Witt ring for $G$ and $Y$ a saturated set of signatures of $R$. Then $I(Y)$ is the union of all $\operatorname{Ann}_{R}(P)$ where $P$ is an m-fold Pfister element $\Pi_{1}^{m}\left(1+\bar{t}_{i}^{\prime}\right)$, $t_{i}^{\prime}$ in $T=\Gamma(Y)$, $m \geqq m_{0}$ for a fixed natural number $m_{0} \geqq 1$.

Proof. Since $Y$ is saturated, we have $Y=V(\Gamma(Y))$. Then by [8, Prop. 1.8(ii)], $I(Y)$ is the radical of $a(\Gamma(Y))$. But then the proof of $[15$, Lem. 4.17] carries over verbatim to the case of a Witt ring for $G$ to yield Lemma 2.18.

CoROllaRy 2.19. If $r_{1}, r_{2}$ are two elements of a Witt ring $R$ for $G$ and $Y$ a saturated set of signatures of $R$, then if $r_{1} \equiv r_{2} \bmod$ $I(Y)$ there exists a Pfister element $P=\Pi_{1}^{m}\left(1+\bar{t}_{i}^{\prime}\right)=\sum_{1}^{2^{m}} \bar{d}_{i}^{\prime}, t_{i}^{\prime}$ in $\Gamma(Y)$ such that $r_{1} P=r_{2} P . \quad$ If $r_{1}=\sum_{1}^{n} \bar{g}_{i}^{\prime}, \operatorname{dim} r_{1}=n$, and $\operatorname{dim} r_{1}>$ $\operatorname{dim} r_{2}$ then $\sum_{i=1}^{n} \sum_{j=1}^{2 m} g_{i}^{\prime} d_{j}^{\prime}$ is isotropic for $R$.

Proof. The first part is clear from Lemma 2.18, while the second part follows from Definitions 1.1 and 1.3 and the equality $r_{1} P=r_{2} P$ in $R$.

In [8, Def. 1.18] a Witt ring $R$ for $G$ was called dimensional if for all elements $r$ of $R$ and natural numbers $s$, we have $\operatorname{dim} s r=$ $s \operatorname{dim} r$.

Proposition 2.20. Let $R$ be a succinct Witt ring for $G$ and $Y$ any saturated set of signatures of $R$. Then $R / I(Y)$ is a dimensional Witt ring for $R$.

Proof. Since $Y=V(\Gamma(Y))$ [8, Prop. 1.8 and 14, Rem. 3.13(ii)] show that $R / I(Y)=\bar{R}$ is a Witt ring for $G$. Let $\sum_{1}^{n} g_{i}^{\prime}$ be anisotropic for $\bar{R}$ but suppose $\sum_{1}^{n} s g_{i}^{\prime}$ is isotropic for $\bar{R}$, where $s$ is a natural number. By Corollary 2.19 there is then a Pfister element $P=\Pi_{1}^{m}\left(1+\bar{t}_{i}^{\prime}\right)=\sum_{1}^{2^{m}} \bar{d}_{i}^{\prime}$ with $t_{i}^{\prime}$ in $\Gamma(Y)$ such that $\sum_{i=1}^{n} \sum_{j=1}^{2^{m}} s g_{i}^{\prime} d_{j}^{\prime}$ is 
isotropic for $R$. Now, for all $\sigma$ in $Y$ we have $\sigma(P)=2^{m}$, hence $\sigma\left(\bar{d}_{j}^{\prime}\right)=1$, for all $\sigma$ in $Y$, i.e., $d_{j}^{\prime}$ is in $\Gamma(Y), j=1, \cdots, 2^{m}$. Since $R$ is succinct, there then exist $\widetilde{t}_{1}^{\prime}, \cdots, \widetilde{t}_{n}^{\prime}$ in $\Gamma(Y)$ such that $\sum_{1}^{n} g_{i}^{\prime} \widetilde{t}_{i}^{\prime}$ is isotropic for $R$. But clearly $\sum_{1}^{n} \bar{g}_{i}^{\prime} \overline{\tilde{t}}_{i}^{\prime} \equiv \sum_{1}^{n} \bar{g}_{i}^{\prime} \bmod I(Y)$ and so $\sum_{1}^{n} g_{i}^{\prime}$ would be isotropic for $\bar{R}$, a contradiction. Hence for all natural numbers $s$, the element $\sum_{1}^{n} s g_{i}^{\prime}$ of $Z[G]$ is anisotropic for $\bar{R}$ so that $\bar{R}$ is dimensional.

CoRollary 2.21. Let $R$ be a representational Witt ring for $G$ and $Y$ a saturated set of signatures of $R$. Then $R / I(Y)$ is dimensional.

Proof. This is immediate from Proposition 2.20 and Theorem 2.11 .

REMARK. If $R$ is a reduced Witt ring for $G$, then $I(X(R))=0$. Therefore a succinct or representational reduced Witt ring is dimensional.

Proposition 2.22. Let $R$ be a Witt ring for $G$ and $Y$ a nonempty set of signatures for $R$. Then if $Y$ is closed in the Zariski topology of $R$ and $I(Y)=\mathfrak{a}(\Gamma(Y))$ then $Y$ is saturated. If $R$ is succinct (or representational), then $Y$ is saturated if and only if $I(Y)=\mathfrak{a}(\Gamma(Y))$ and $Y$ is closed.

Proof. From the definition of the Zariski topology the closure of $Y=\left\{\sigma_{1}\right.$ in $\left.X(R) \mid \operatorname{ker} \sigma_{1} \supset I(Y)\right\}$. Now if $I(Y)=\mathfrak{a}(\Gamma(Y))$ and $\sigma$ lies in $V(\Gamma(Y))$ then clearly $\operatorname{ker} \sigma \supset a(\Gamma(Y))=I(Y)$, so that if $Y$ is closed also, $\sigma$ is in $Y$. Hence $V(\Gamma(Y)) \subset Y$. Since the opposite inclusion is always true, $Y$ is saturated.

Now suppose $R$ is succinct and $Y$ is saturated. Let $r$ be in $I(Y)$. We shall show by induction on $\operatorname{dim} r$ that $r$ lies in $\mathfrak{a}(\Gamma(Y)$ ). By Lemma 2.18 there exists a Pfister element $P=\Pi_{1}^{m}\left(1+\bar{t}_{i}^{\prime}\right)=$ $\sum_{1}^{2^{m}} \bar{d}_{i}^{\prime}$ with $t_{i}^{\prime}$ and $d_{i}^{\prime}$ in $T=\Gamma(Y)$, and $r P=0$. If $\operatorname{dim} r=1$, then $r$ is a unit in $R$, so that this case is impossible. If $\operatorname{dim} r=2$, then $r=\bar{g}_{1}^{\prime}+\bar{g}_{2}^{\prime}$ and so $\bar{g}_{1}^{\prime} P=-\bar{g}_{2}^{\prime} P$ or $P=-\bar{g}_{1}^{\prime} \bar{g}_{2}^{\prime} P$. Now for all $\sigma$ in $Y$ we have $\sigma(P)=2^{m}$. Hence for all $\sigma$ in $Y$, we have $2^{m}=\sigma\left(-\bar{g}_{1}^{\prime} \bar{g}_{2}^{\prime}\right) 2^{m}$ or $\sigma\left(-\bar{g}_{1}^{\prime} \bar{g}_{2}^{\prime}\right)=1$, i.e., $t=-g_{1}^{\prime} g_{2}^{\prime}$ is in $T$ and $\bar{g}_{1}^{\prime}+\bar{g}_{2}^{\prime}=\bar{g}_{1}^{\prime}(1-\bar{t})$ is in $a(T)$. Suppose now all $r$ in $I(Y)$ with $\operatorname{dim} r<n$ lie in $a(T)$. Let $r=\sum_{1}^{n} \bar{g}_{i}^{\prime}$ and $\operatorname{dim} r=n$. Then $r P=0$ forces $\sum_{i=1}^{n} \sum_{j=1}^{2^{m}} g_{i}^{\prime} d_{j}^{\prime}$ to be isotropic for $R$. Since $R$ is succinct (or if $R$ is representational, by Theorem 2.11) there exist $\widetilde{t}_{1}^{\prime}, \cdots, \widetilde{t}_{n}^{\prime}$ in $T$ with $\sum_{1}^{n} g_{i}^{\prime} \widetilde{t}_{i}^{\prime}$ isotropic for $R$. Now $r=\sum_{1}^{n} \bar{g}_{i}^{\prime}\left(1-{\overline{\tilde{t}_{i}^{\prime}}}_{i}^{\prime}+\sum_{1}^{n} \bar{g}_{i}^{\prime} \overline{\tilde{t}}_{i}^{\prime}\right.$ and since $\sigma(r)=\sigma\left(\sum_{1}^{n} \bar{g}_{i}^{\prime}{\overline{\tilde{t}_{i}}}_{i}^{\prime}\right)$ for all $\sigma$ in $Y$, 
the element $\sum_{1}^{n} \bar{g}_{i}^{\prime} \overline{\vec{t}}_{i}^{\prime}$ is in $I(Y)$ and by induction assumption, in $\mathfrak{a}(T)$. Since $r \equiv \sum_{1}^{n} \bar{g}_{i}^{\prime} \overline{\tilde{t}}_{i}^{\prime} \bmod a(T)$, the element $r$ is in $a(T)$ also, and we have proved that $I(Y) \subset \mathfrak{a}(T)$. The opposite inclusion is clear so that $I(Y)=\mathfrak{a}(T)$.

Finally since $Y=V(\Gamma(Y))$ it is clear that $Y= \begin{cases}\sigma_{1} & \text { in }\end{cases}$ $\left.X(R) \mid \sigma_{1}(T)=1\right\}=\left\{\sigma_{1}\right.$ in $\left.X(R) \mid \operatorname{ker} \sigma_{1} \supset\{1-t\}\right\}=\left\{\sigma_{1}\right.$ in $X(R) \mid \operatorname{ker} \sigma_{1} \supset$ $I(Y)\}$ which means that $Y$ is closed.

CoROLlaRY 2.23. Let $R$ be a succinct or representational Witt ring for $G$ such that $X(R) \neq \varnothing$. Then the torsion subgroup of $R$, $R_{t}=\mathfrak{a}(\Gamma(X(R)))$, i.e., $R_{t}$ is generated by $1-\bar{t}^{\prime}$ with $\sigma\left(\bar{t}^{\prime}\right)=1$ for all $\sigma$ in $X(R)$.

Proof. By [14, Thm. 3.9(v) and Prop. 3.15] $R_{t}=I(X(R))$. Since $V(\Gamma(X(R)))=X(R)$, Proposition 2.22 applies with $Y=X(R)$ and yields the result.

Proposition 2.24. (cf. [17, Thm. 2.2; 18, Thm. 2.2]). Let $R$ be a representational Witt ring for $G$ and $Y$ a saturated set of signatures of $R$. Then $\bar{R}=R / I(Y)$ is again representational.

Proof. Let $\bar{r}_{i}$ be elements of $\bar{R}$ with $\operatorname{dim}_{\bar{l}} \bar{r}_{i}=n_{i}, i=1,2$, and suppose $\operatorname{dim}_{\overline{1 l}}\left(\bar{r}_{1}+\bar{r}_{2}\right)<n_{1}+n_{2}$. Let $\sum_{j=1}^{n_{i}} g_{i j}^{\prime}$ be anisotropic representations of $\bar{r}_{i}$ and let $r_{i}=\sum_{j=1}^{n_{i}} \bar{g}_{i j}^{\prime}$ in $R$. By Definition 1.1, $\operatorname{dim}_{\bar{l}} \bar{r}_{\imath} \leqq$ $\operatorname{dim}_{R} r_{i} \leqq n_{i}$, so that $\operatorname{dim}_{R} r_{i}=n_{i}$ too. By Corollary 2.19 there exists an $m$-fold Pfister element $P=\prod_{1}^{m}\left(1+\bar{t}_{i}^{\prime}\right)=\sum_{1}^{2^{m}} \bar{d}_{i}^{\prime}, t_{i}^{\prime}, d_{i}^{\prime}$ in $\Gamma(Y)$ with $\operatorname{dim}_{R}\left(r_{1} P+r_{2} P\right)<2^{m}\left(n_{1}+n_{2}\right)$. Now in $R$ we have $\sigma\left(r_{i} P\right)=$ $\sigma\left(2^{m} r_{i}\right)$ for all $\sigma$ in $Y$. Hence $\bar{r}_{i} \bar{P}=2^{m} \bar{r}_{i}$ in $\bar{R}$, where $\bar{P}$ denotes the image of $P$ in $\bar{R}$. By Corollary 2.21 the ring $\bar{R}$ is dimensional, so that $2^{m} n_{i}=\operatorname{dim}_{\bar{k}}\left(\bar{r}_{i} \bar{P}\right) \leqq \operatorname{dim}_{R} r_{i} P \leqq 2^{m} n_{i}$. Since $R$ is representational, Proposition 2.4 yields $g^{\prime}$ in $G^{\prime}$ with $g^{\prime}$ in $D\left(r_{1} P\right)$ and $-g^{\prime}$ in $D\left(r_{2} P\right)$. From Definition 1.2, it is clear that $g^{\prime}$ is in $D_{\bar{R}}\left(\bar{r}_{1} \bar{P}\right)=D_{\bar{R}}\left(2^{m} \bar{r}_{1}\right)$ and $-g^{\prime}$ is in $D_{\bar{R}}\left(\bar{r}_{2} \bar{P}\right)=D_{\bar{R}}\left(2^{m} \bar{r}_{2}\right)$. But by [8, Thm. 1.17] we have $D_{\bar{R}}\left(2^{m} \bar{r}_{i}\right)=D_{\bar{l}}\left(\bar{r}_{i}\right)$ so that by Proposition 2.4 , the ring $\bar{R}$ is representational.

LeMma 2.25. Let $Y$ be a saturated set of signatures of a Witt ring $R$ for $G$. Then all signatures of $\bar{R}=R / I(Y)$ are induced by signatures in $Y$ and a subset $Y^{\prime} \subset Y$ is saturated as a set of signatures of $\bar{R}$ if and only if it is saturated as a set of signatures of $R$.

Proof. By [8, Lem. 3.5] all signatures of $\bar{R}$ are induced by signatures in $Y$. Let $Y^{\prime}$ be a subset of $Y$ and let $\bar{\Gamma}\left(Y^{\prime}\right)$ denote 
the set of $g^{\prime}$ in $G^{\prime}$ such that $\bar{\sigma}\left(\overline{\bar{g}}^{\prime}\right)=1$ where $\bar{\sigma}$ is the signature of $\bar{R}$ induced by $\sigma$ and $\overline{\bar{g}}^{\prime}$ is the image of $g^{\prime}$ in $\bar{R}$. Clearly $\bar{\sigma}\left(\overline{\bar{g}}^{\prime}\right)=\sigma\left(\bar{g}^{\prime}\right)$ so that $\bar{\Gamma}\left(Y^{\prime}\right)=\Gamma\left(Y^{\prime}\right)$. Now $V_{R}\left(\Gamma\left(Y^{\prime}\right)\right) \subset V_{R}(\Gamma(Y))=Y$ so $Y^{\prime}=$ $V_{R}\left(\Gamma\left(Y^{\prime}\right)\right)$ if and only if $\bar{Y}^{\prime}=V_{\bar{R}}\left(\Gamma\left(\bar{Y}^{\prime}\right)\right)$ where $\bar{Y}^{\prime}$ denotes the signatures of $\bar{R}$ induced by the signatures in $Y^{\prime}$.

Proposition 2.26. Let $R$ be a succinct Witt ring for $G$ and $Y$ a saturated set of signatures of $R$. Then $\bar{R}=R / I(Y)$ is again succinct.

Proof. Let $\bar{Y}^{\prime}$ be a saturated set of signatures of $\bar{R}$. By Lemma 2.25, the lifted set of signatures $Y^{\prime}$ of $R$ is again saturated. Suppose now for $g_{i}^{\prime}$ in $G^{\prime}, i=1, \cdots, n$, and $t_{i j}^{\prime}, j=1, \cdots, m_{i}$ in $\Gamma\left(Y^{\prime}\right)=\Gamma\left(\bar{Y}^{\prime}\right) \supset \Gamma(Y)$ the element $\sum_{i=1}^{n} \sum_{j=1}^{m_{i}} g_{i}^{\prime} t_{i j}^{\prime}$ is isotropic for $\bar{R}$. Then by Corollary 2.19 there is a Pfister element $P=\Pi\left(1+\bar{s}_{k}^{\prime}\right)=\sum \bar{d}_{k}^{\prime}$ in $R$ with $s_{k}^{\prime}$ and $d_{k}^{\prime}$ in $\Gamma(Y)$ such that $\sum_{i} \sum_{j} \sum_{k} g_{i}^{\prime} t_{i j}^{\prime} d_{k}^{\prime}$ is isotropic for $R$. Since by Lemma 2.25 , the set $Y^{\prime}$ is also saturated as a subset of $X(R)$ and all $t_{i j}^{\prime} d_{k}^{\prime}$ lie in $\Gamma\left(Y^{\prime}\right)$, the fact that $R$ is succinct then yields elements $t_{1}^{\prime}, \cdots, t_{n}^{\prime}$ in $\Gamma\left(Y^{\prime}\right)$ with $\sum_{1}^{n} g_{i}^{\prime} t_{i}^{\prime}$ isotropic for $R$. But then clearly $\sum_{1}^{n} g_{i}^{\prime} t_{i}^{\prime}$ is also isotropic for $\bar{R}$ and since $\Gamma\left(Y^{\prime}\right)=$ $\Gamma\left(\bar{Y}^{\prime}\right)$, Proposition 2.26 is proved.

Finally, we show that the definitions $O_{4}$ of [17 and 18] and our concept of representational coincide for reduced Witt rings. We begin with

Definitions 2.27. (i) Let $R$ be a Witt ring for $G$. For $r=$ $\sum_{1}^{n} \bar{g}_{i}^{\prime}$ in $R$, let $M_{n}(r)=\left\{h^{\prime}\right.$ in $G^{\prime} \mid$ there exist $h_{2}^{\prime}, \cdots, h_{n}^{\prime}$ in $G^{\prime}$ with $\left.r=\bar{h}^{\prime}+\sum_{2}^{n} \bar{h}_{i}^{\prime}\right\}$. Thus if $n=\operatorname{dim} r$, we have $M_{n}(r)=D(r)$ by Definition 1.2.

(ii) $R$ is said to satisfy $O_{4}$ (cf. [17, Introduction]) if given $r_{i}=\sum_{j=1}^{n_{i}} \bar{g}_{i j}^{\prime}, i=1,2$, in $R$, with $g^{\prime}$ in $M_{n_{1}+n_{2}}\left(r_{1}+r_{2}\right)$ there exist $g_{i}^{\prime}$ in $M_{n_{i}}\left(r_{i}\right), i=1$, 2, with $g^{\prime}$ in $M_{2}\left(\bar{g}_{1}^{\prime}+\bar{g}_{2}^{\prime}\right)$.

LEMMA 2.28. Let $R$ be a Witt ring for $G$ and $r=\sum_{1}^{n} \bar{g}_{i}^{\prime}$ an element of $R$ with $\operatorname{dim} r<n$. Then $M_{n}(r)=G^{\prime}$.

Proof. We may write, by Definition 1.1, $r=\sum_{1}^{m} \bar{h}_{i}^{\prime}$ with $m<n$. By [8, Rem. 1.24, Lem.], $n-m$ is even so that for any $g^{\prime}$ in $G^{\prime}$ we have $r=\sum_{1}^{m} \bar{h}_{i}^{\prime}+((n-m) / 2)\left(\bar{g}^{\prime}-\bar{g}^{\prime}\right)$ and by Definition 2.27(i), $g^{\prime}$ lies in $M_{n}(r)$.

Proposition 2.29. Let $R$ be a Witt ring for $G$. Then $R$ is representational if and only if it satisfies $O_{4}$.

Proof. Let $r_{i}, i=1,2$, be elements of $R$ that can be written 
as the sum of $n_{i}, i=1,2$, elements of $\bar{G}^{\prime}$. Suppose first that $R$ satisfies $O_{4}$. Assume $n_{i}=\operatorname{dim} r_{i}$ and $\operatorname{dim}\left(r_{1}+r_{2}\right)=n_{1}+n_{2}$, so that by Definition 2.27 (i) we have $M_{n_{i}}\left(r_{i}\right)=D\left(r_{i}\right)$ and $M_{n_{1}+n_{2}}\left(r_{1}+r_{2}\right)=$ $D\left(r_{1}+r_{2}\right)$. Hence for $g^{\prime}$ in $D\left(r_{1}+r_{2}\right)$ there exist $g_{i}^{\prime}$ in $D\left(r_{i}\right), i=1,2$, with $g^{\prime}$ in $M_{2}\left(\bar{g}_{1}^{\prime}+\bar{g}_{2}^{\prime}\right)$. By Lemma 2.3, $\bar{g}_{1}^{\prime}+\bar{g}_{2}^{\prime}$ has dimension two so that $M_{2}\left(\bar{g}_{1}^{\prime}+\bar{g}_{2}^{\prime}\right)=D\left(\bar{g}_{1}^{\prime}+\bar{g}_{2}^{\prime}\right)$ and $R$ is representational.

Suppose now that $R$ is representational. Then if $n_{i}=\operatorname{dim} r_{i}$, $i=1,2$, and $\operatorname{dim}\left(r_{1}+r_{2}\right)=n_{1}+n_{2}$ we see immediately that $O_{4}$ holds for all elements of $M_{n_{1}+n_{2}}\left(r_{1}+r_{2}\right)$. Next, still supposing $n_{i}=\operatorname{dim} r_{i}$, $i=1$, 2, let $\operatorname{dim}\left(r_{1}+r_{2}\right)<n_{1}+n_{2}$. By Lemma 2.28, $M_{n_{1}+n_{2}}\left(r_{1}+r_{2}\right)=G^{\prime}$ and by Proposition 2.4 there is a $g^{\prime}$ in $G^{\prime}$ with $g^{\prime}$ in $D\left(r_{1}\right)$ and $-g^{\prime}$ in $D\left(r_{2}\right)$. But then we have $G^{\prime}=M_{2}\left(\bar{g}^{\prime}+\left(-\bar{g}^{\prime}\right)\right)$, again by Lemma 2.28 , so $O_{4}$ is true for all elements of $M_{n_{1}+n_{2}}\left(r_{1}+r_{2}\right)$.

Finally, if $n_{1}>\operatorname{dim} r_{1}$, then $G^{\prime}=M_{n_{1}+n_{2}}\left(r_{1}+r_{2}\right)=M_{n_{1}}\left(r_{1}\right)$ and any $g^{\prime}$ in $G^{\prime}$ is in $M_{2}\left(\bar{g}^{\prime}+\bar{g}_{2}^{\prime}\right)$ where $g_{2}^{\prime}$ is any element of $M_{n_{2}}\left(r_{2}\right)$, so that $\mathrm{O}_{4}$ holds here also.

REMARK 2.30. Let $R$ be a reduced Witt ring for $G$ and $X(R)$ its set of signatures. By Remark 1.6 we have $r_{1}=r_{2}$ in $R$ if and only if $\sigma\left(r_{1}\right)=\sigma\left(r_{2}\right)$ for all $\sigma$ in $X(R)$. For each $\sigma$ in $X(R)$ we define a character $\chi$ of $G^{\prime} / \Gamma(X(R))$ by $\chi\left(g^{\prime} \Gamma(X(R))\right)=\sigma\left(\bar{g}^{\prime}\right)$ and denote this set of characters by $X$. Now it can be verified that the Zariski topology of the set of minimal prime ideals of $Z[G]$ induces the usual topology used in Pontryagin duality on the character group of $G^{\prime} / \Gamma(X(R))$. Since $X(R)$ corresponds to all minimal prime ideals of $Z[G]$ containing the ideal $K$, the set $X(R)$ is closed in the Zariski topology and so $X$ is closed in the character group of $G^{\prime} / \Gamma(X(R))$. Thus the pair $\left(X, G^{\prime} / \Gamma(X(R))\right)$ satisfies $O_{1}, O_{2}, O_{3}$ of [17]. Now by definition, the pair $\left(X, G^{\prime} /(\Gamma X(R))\right)$ satisfies $O_{4}$ of [17] if and only if $R$ satisfies $O_{4}$ of Definition 2.27(ii) or, by Proposition 2.29, if and only if $R$ is representational. Thus if $R$ is a representational reduced Witt ring for $G$, then $\left(X, G^{\prime} / \Gamma(X(R))\right)$ is a space of orderings as defined in [17].

We point out explicitly that if $R$ is a reduced representational Witt ring our Proposition 2.24 is now seen to be equivalent to [18, Thm. 2.2].

3. Applications to succinct and representational Witt rings. In this section we show how some of the results proved in [8] for $R=W(C) / I(Y)$ can be carried over to succinct Witt rings for $G$. Here $C$ is a connected semilocal ring with every residue class field containing at least 3 elements, $W(C)$ is the Witt ring of classes of symmetric nondegenerate bilinear $C$-forms, and $Y$ is a saturated set of signatures of $W(C)$. We also give a version of 
some of the results of [20, Thm. 2.2] and [13, Thm. 1] for Witt rings $R$ for $G$ with $R / I(X(R))$ representational.

LEMMA 3.1. Let $R$ be a succinct Witt ring for $G, Y$ a saturated set of signatures of $R$, and $\bar{R}=R / I(Y)$. Then an element $\sum_{1}^{n} g_{i}^{\prime}$ of $Z[G]$ is isotropic for $\bar{R}$ if and only if there exist $t_{1}^{\prime}, \cdots, t_{n}^{\prime}$ in $\Gamma(Y)$ with $\sum_{1}^{n} g_{i}^{\prime} t_{i}^{\prime}$ isotropic for $R$.

Proof. Since $\sum_{1}^{n} \bar{g}_{i}^{\prime} \bar{t}_{i}^{\prime} \equiv \sum_{1}^{n} \bar{g}_{i}^{\prime} \bmod I(Y)$ it is clear that if $\sum_{1}^{n} g_{i}^{\prime} t_{i}^{\prime}$ is isotropic for $R$ then $\sum_{1}^{n} g_{i}^{\prime}$ is isotropic for $\bar{R}$. Conversely, if $\sum_{1}^{n} g_{i}^{\prime}$ is isotropic for $\bar{R}$, there exists by Corollary 2.19 a Pfister element $P=\Pi_{1}^{m}\left(1+\bar{s}_{j}^{\prime}\right)=\sum_{1}^{2^{m}} \bar{d}_{j}^{\prime}$ of $R$ with $s_{j}^{\prime}, d_{j}^{\prime}$ in $\Gamma(Y)$, such that $\sum_{i=1}^{n} \sum_{j=1}^{2^{m}} g_{i}^{\prime} d_{j}^{\prime}$ is isotropic for $R$. Since $R$ is succinct there then exist $t_{1}^{\prime}, \cdots, t_{n}^{\prime}$ in $\Gamma(Y)$ with $\sum_{1}^{n} g_{i}^{\prime} t_{i}^{\prime}$ isotropic for $R$.

Definitions 3.2. Let $R$ be a Witt ring for $G$, and $A, T$ subsets of $G^{\prime}$.

(i) $T$ is called saturated if $T=\Gamma(V(T))$ with the notations of Definition 1.7.

(ii) The pair $(A, T)$ is said to be anisotropic for $R$ if all finite sums $\sum a_{i} t_{i}$ of $Z[G]$ with, not necessarily distinct, $a_{i}$ in $A, t_{i}$ in $T$, are anisotropic for $R$.

(iii) $D_{T}(A)=\left\{g^{\prime}\right.$ in $G^{\prime} \mid g^{\prime}$ in $D\left(\sum_{1}^{n} \bar{a}_{i} \bar{t}_{i}\right)$ for some, not necessarily distinct, $a_{i}$ in $A, t_{i}$ in $T$, and arbitrary $\left.n\right\}$.

(iv) Denote by $Z(A, T)$ the set of all semisignatures (Definition 1.8) $\tau$ of $R$ which are constant on the cosets of $\bar{T}$ in $\bar{G}^{\prime}$ and with $\tau(\bar{a})=1$ for all $a$ in $A$.

THEOREM 3.3. Let $R$ be a succinct Witt ring for G, $T$ a saturated subset of $G^{\prime}$, and $A$ an arbitrary subset of $G^{\prime}$.

(i) The pair $(A, T)$ is anisotropic for $R$ if and only if $Z(A, T) \neq \varnothing$.

(ii) If $Z(A, T) \neq \varnothing$ then $D_{T}(A)=\bigcap_{\tau i n Z(A, T)} \tau^{-1}(1)$.

Proof. (i) Let $Y=V(T)$. Since $T=\Gamma(V(T)$ ) we have $V(\Gamma(Y))=V(\Gamma(V(T)))=Y$, so that $Y$ is also saturated. Now let $\bar{R}=R / I(Y)$ and for $g^{\prime}$ in $G^{\prime}$ denote its image in $\bar{R}$ by $\overline{\bar{g}}^{\prime}$. By Proposition 2.20 the Witt ring $\bar{R}$ is dimensional and by Lemma 3.1 all finite sums $\left\{\sum a_{i} \mid a_{i}\right.$ in $\left.A\right\}$ are anisotropic for $\bar{R}$ if and only if the pair $(A, T)$ is anisotropic for $R$. But this condition on $A$ yields by [8, Th. 1.17] a semisignature $\bar{\tau}$ of $\bar{R}$ with $\tau(\overline{\bar{a}})=1$ for all $a$ in $A$. Now for all $t$ in $T$, we have $\sigma\left(\bar{g}^{\prime}-\bar{g}^{\prime} \bar{t}\right)=0$ for all $\sigma$ in $Y$, so that $\bar{g}^{\prime} \equiv \bar{g}^{\prime} \bar{t} \bmod I(Y)$. Hence $\tau$, the lifted semisignature of $\bar{\tau}$ on $R$, is constant on cosets of $\bar{T}$ in $\bar{G}^{\prime}$ and clearly $\tau(\bar{a})=1$ for all $a$ in $A$. 
Conversely, let $\tau$ be in $Z(A, T)$. Then, clearly $\tau(\mathfrak{a}(T))=0$. By Proposition 2.22, the ideal $\mathfrak{a}(T)=I(Y)$, so that $\tau$ induces a semisignature $\bar{\tau}$ on $\bar{R}$. Since for all $a_{i}$ in $A$ we have $\bar{\tau}\left(\sum_{1}^{n} \overline{\bar{a}}_{i}\right)=n$ we must have $\sum_{1}^{n} a_{i}$ anisotropic for $\bar{R}$, which again by Lemma 3.1 means that the pair $(A, T)$ is anisotropic for $R$.

(ii) For $g^{\prime}$ in $D_{T}(A)$, there exists $a_{i}$ in $A, t_{i}$ in $T, i=1, \cdots, n$, with $g^{\prime}$ in $D\left(\sum_{1}^{n} \bar{a}_{i} \bar{t}_{i}\right)$. Now for all $\tau$ in $Z(A, T)$ we have $\tau\left(\sum_{1}^{n} \bar{a}_{i} \bar{t}_{i}\right)$ $n=\operatorname{dim} \sum_{1}^{n} \bar{a}_{i} \bar{t}_{i}$, so that by Lemma 2.9, we have $\tau\left(\bar{g}^{\prime}\right)=1$. Thus $D_{T}(A) \subset \bigcap_{\text {in } Z(A, T)} \tau^{-1}(1)$.

Now if $g^{\prime}$ is not in $D_{T}(A)$ then $\left(A \cup\left\{-g^{\prime}\right\}, T\right)$ is anisotropic for $R$ by Lemma 1.4. Thus by (i) $Z\left(A \cup\left\{-g^{\prime}\right\}, T\right) \neq \varnothing$ and there exists a semisignature $\tau$ constant on cosets of $\bar{T}$ in $\bar{G}^{\prime}$ with $\tau\left(-\bar{g}^{\prime}\right)=$ $\tau(\bar{a})=1$ for all $a$ in $A$. Thus $\tau$ is in $Z(A, T)$ but $g^{\prime}$ is not in $\tau^{-1}(1)$. Consequently, $D_{T}(A) \supset \bigcap_{\tau \operatorname{in} Z(A, T)} \tau^{-1}(1)$, completing the proof.

Definition 3.4. Let $R$ be a Witt ring for $G$ and $r$ an element of $R$. Then $r$ is weakly isotropic if there exists a natural number $m$ with $\operatorname{dim}(m r)<m \operatorname{dim} r$.

Lemma 3.5. Let $R$ be a succinct Witt ring for $G$. An element $r=\sum_{1}^{n} \bar{g}_{i}^{\prime}$ of $R$ with $n=\operatorname{dim} r$ is weakly isotropic if and only if there exist $t_{1}^{\prime}, \cdots, t_{n}^{\prime}$ in $\Gamma(X(R))$ such that $\sum_{1}^{n} g_{i}^{\prime} t_{i}^{\prime}$ is isotropic for $R$.

Proof. If $r$ is weakly isotropic then for some natural number $m$ the element $\sum_{1}^{n} m g_{i}^{\prime}$ is isotropic for $R$, i.e., $\sum_{i=1}^{n} \sum_{1}^{m} g_{i}^{\prime} \cdot 1$ is isotropic for $R$. Since 1 lies in $\Gamma(X(R))$ and $R$ is succinct there then exist $t_{1}^{\prime}, \cdots, t_{n}^{\prime}$ in $\Gamma(X(R))$ with $\sum_{1}^{n} g_{i}^{\prime} t_{i}^{\prime}$ isotropic for $R$.

Conversely, if there exist $t_{1}^{\prime}, \cdots, t_{n}^{\prime}$ in $\Gamma(X(R))$ with $\sum_{1}^{n} g_{i}^{\prime} t_{i}^{\prime}$ isotropic for $R$, then $r \equiv \sum_{1}^{n} \bar{g}_{i}^{\prime} \bar{t}_{i}^{\prime}=\sum_{1}^{l} \bar{h}_{i}^{\prime} \bmod I(X(R))$ with $l<n$. By Corollary 2.19 then, there is a Pfister element $P=\Pi_{1}^{m}\left(1+\bar{s}_{i}^{\prime}\right)=\sum_{1}^{2^{m}} \bar{d}_{i}^{\prime}$ of $R$ with $s_{i}^{\prime}, d_{i}^{\prime}$ in $\Gamma(X(R))$ with $\operatorname{dim} r P<n 2^{m}$. Now for all $\sigma$ in $X(R)$, we have $\sigma(r P)=2^{m} \sigma(r)=\sigma\left(2^{m} r\right)$. Thus $r P-2^{m} r$ is a nilpotent element of $R$. By [14, Prop. 3.15] then there exists a natural number $s$ such that $s\left(r P-2^{m} r\right)=0$, or $s r P=2^{m} s r$. But then $\operatorname{dim} 2^{m} s r=\operatorname{dim} s P r<2^{m} s n$ so that $r$ is weakly isotropic.

THEOREM 3.6. Let $R$ be a succinct Witt ring for $G, Y$ a saturated set of signatures of $R, T=\Gamma(Y)$, and $r=\sum_{1}^{n} \bar{g}_{i}^{\prime}$ an element of $R$ with $\operatorname{dim} r=n$. Then if for all semisignatures $\tau$ of $R$ constant on cosets of $\bar{T}$ in $\bar{G}^{\prime}$ we have $|\tau(r)|<n$, there exist $t_{1}^{\prime}, \cdots, t_{n}^{\prime}$ in $T$ with $\sum_{1}^{n} g_{i}^{\prime} t_{i}^{\prime}$ isotropic for $R$.

Proof. Let $\bar{R}=R / I(Y)$ and $\bar{r}$ be the image of $r$ in $\bar{R}$. Then by [8, Prop. 1.25] we have $\operatorname{dim}_{\bar{R}}(\bar{r})<\operatorname{dim}_{R} r=n$ since by Proposition 
2.20 the ring $\bar{R}$ is dimensional. Thus $\sum_{1}^{n} g_{i}^{\prime}$ is isotropic for $\bar{R}$, and so by Lemma 3.1 , there exist $t_{1}^{\prime}, \cdots, t_{n}^{\prime}$ in $T$ such that $\sum_{1}^{n} g_{i}^{\prime} t_{i}^{\prime}$ is isotropic for $R$.

CoROllaRy 3.7. Let $R$ be a succinct Witt ring for $G$ and $r$ an element of $R$ with $\operatorname{dim} r=n$. If for all semisignatures $\tau$ of $R$ we have $|\tau(r)|<n$, then $r$ is weakly isotropic.

Proof. By [8, Rem. 1.10(i)], Theorem 3.6 is applicable to $Y=$ $X(R)$. Hence, if $r=\sum_{1}^{n} \bar{g}_{i}^{\prime}$, there exist $t_{1}^{\prime}, \cdots, t_{n}^{\prime}$ in $\Gamma(X(R))$ with $\sum_{1}^{n} g_{i}^{\prime} t_{i}^{\prime}$ isotropic for $R$. Lemma 3.5 then completes the proof.

DEFinitions 3.8. Let $R$ be a Witt ring for $G$ and consider $X=X(R)$ in the Zariski topology of $R$.

(i) $R$ satisfies SAP if every clopen (closed and open) subset of $X$ is of the form $V\left(g^{\prime}\right)$ for $g^{\prime}$ in $G^{\prime}$.

(ii) $R$ satisfies WAP if the family of clopen subsets $\left\{V\left(g^{\prime}\right) \mid g^{\prime}\right.$ in $G^{\prime}$ \} forms a basis of the topology of $X$.

THeOREM 3.9. (cf. [20, Thm. 2.2; 13, Thm. 1]). Let $R$ be a Witt ring for $G$ with $\bar{R}=R / I(X(R))$ representational. Then SAP and WAP are equivalent.

Proof. It is clear that $\mathrm{SAP} \Rightarrow \mathrm{WAP}$.

$\mathrm{WAP} \Rightarrow \mathrm{SAP}$. Just as in the beginning of the proof of $[20$, Thm. 2.2] it suffices to show that if $Y=\bigcap_{1}^{n} V\left(g_{i}^{\prime}\right)=\bigcup_{1}^{n} V\left(h_{i}^{\prime}\right)$ there exists a $g^{\prime}$ in $G^{\prime}$ with $V\left(g^{\prime}\right)=Y$. Let $r_{1}=\Pi_{1}^{n}\left(1+\bar{g}_{i}^{\prime}\right), r_{2}=\Pi_{1}^{n}(1-$ $\left.\bar{h}_{i}^{\prime}\right)$. Then

$$
\begin{array}{lll}
\sigma\left(r_{1}\right)=2^{n}, \sigma\left(r_{2}\right)=0 & \text { for } & \sigma \text { in } Y \\
\sigma\left(r_{1}\right)=0, \sigma\left(r_{2}\right)=2^{n} & \text { for } & \sigma \text { in } X-Y .
\end{array}
$$

Thus for all $\sigma$ in $X(R)$, we have $\sigma\left(r_{1}+r_{2}\right)=2^{n}=\sigma\left(2^{n}\right)$. Hence if $\bar{r}$ denotes the image of $r$ in $\bar{R}$ and $\bar{\sigma}$ the signature of $\bar{R}$ induced by $\sigma$, we have $\bar{\sigma}\left(\bar{r}_{1}+\bar{r}_{2}-2^{n}\right)=0$ for all $\sigma$ in $X(R)=X(\bar{R})$. But in $\bar{R}$ we clearly have $I(X(\bar{R}))=0$ so that $\bar{r}_{1}+\bar{r}_{2}=2^{n}=\sum_{1}^{2^{n}} 1$. Hence $\operatorname{dim}_{\bar{R}}\left(\bar{r}_{1}+\bar{r}_{2}\right) \leqq 2^{n}$. On the other hand from (3.10) it is clear that since $\bar{\sigma}(\bar{r})=\sigma(r)$, we have $\operatorname{dim}_{\bar{R}} \bar{r}_{i}=2^{n} i=1,2$. Thus by Proposition 2.4, there is a $g^{\prime}$ in $G^{\prime}$ with $g^{\prime}$ in $D_{\bar{R}}\left(\bar{r}_{1}\right)$ and $-g^{\prime}$ in $D_{\bar{R}}\left(\bar{r}_{2}\right)$.

Now let $\overline{\bar{g}}^{\prime}$ denote the image of $g^{\prime}$ in $\bar{R}$. Then for all $\sigma$ in $X(R)$ we have $\sigma\left(\bar{g}^{\prime}\right)=\bar{\sigma}\left(\overline{\bar{g}}^{\prime}\right)$. Hence (3.10) coupled with Lemma 2.9 shows that $\sigma\left(\bar{g}^{\prime}\right)=1$ for $\sigma$ in $Y$ and $\sigma\left(\bar{g}^{\prime}\right)=-1$ for $\sigma$ in $X-Y$. Thus $Y=V\left(g^{\prime}\right)$. 
REMARK. The hypothesis that $\bar{R}$ is representational is needed in Theorem 3.9 since it has been noted in [5, §3] that WAP and SAP are not necessarily equivalent for arbitrary Witt rings for $G$.

\section{Strongly representational Witt rings.}

Definition 4.1. A representational Witt ring $R$ for $G$ is said to be strongly representational if for $g_{1}^{\prime}, g_{2}^{\prime}$ in $G^{\prime}$ with $\bar{g}_{1}^{\prime}+\bar{g}_{2}^{\prime} \neq 0$ in $R$ and $g^{\prime}$ in $D\left(\bar{g}_{1}^{\prime}+\bar{g}_{2}^{\prime}\right)$ we have

$$
\bar{g}^{\prime}+\bar{g}^{\prime} \bar{g}_{1}^{\prime} \bar{g}_{2}^{\prime}=\bar{g}_{1}^{\prime}+\bar{g}_{2}^{\prime} .
$$

Proposition 4.2. If $R$ is a reduced Witt ring for $G$ with $X(R) \neq \varnothing$, then $R$ is representational if and only if it is strongly representational.

Proof. One implication is trivial. Suppose now $R$ is representational. If $g^{\prime}$ is in $D\left(\bar{g}_{1}^{\prime}+\bar{g}_{2}^{\prime}\right)$,with $\bar{g}_{1}^{\prime}+\bar{g}_{2}^{\prime} \neq 0$ then by [8, Rem. 1.24, Lem.] there exists an $h^{\prime}$ in $G^{\prime}$ such that $\bar{g}^{\prime}+\bar{h}^{\prime}=\bar{g}_{1}^{\prime}+\bar{g}_{2}^{\prime}$ in $R$. Squaring this leads to $2 \bar{g}_{1}^{\prime} \bar{g}_{2}^{\prime}=2 \bar{g}^{\prime} \bar{h}^{\prime}$ in $R$. As noted in Remark 1.6, the ring $R$ is torsion free so that $\bar{g}_{1}^{\prime} \bar{g}_{2}^{\prime}=\bar{g}^{\prime} \bar{h}^{\prime}$ or $\bar{h}^{\prime}=\bar{g}^{\prime} \bar{g}_{1}^{\prime} \bar{g}_{2}^{\prime}$ which proved Proposition 4.2.

Proposition 4.2 is false in case $R_{t} \neq 0$, cf. Remark 6.14.

We now proceed to record some of the results of [6] and [7] concerning Pfister elements that remain vaild for strongly representational Witt rings for $G$. Most of the proofs of these results are essentially just the proofs in the cited references with some suitable modifications for the abstract situation. We give a fair number of these proofs in detail and then just record further results without proofs since these can be supplied by the (willing) reader on the basis of the literature referred to and the modifications made in the earlier proofs.

Lemma 4.3. (cf. [6, Cor. 1.9, 1.10; 16, Prop. 1.3 p. 276]). Let $R$ be a strongly representational Witt ring for $G$ and let $g_{1}^{\prime}, g_{2}^{\prime}, h^{\prime}$ be elements of $G^{\prime}$.

(i) If $1+\bar{g}_{1}^{\prime} \neq 0$ in $R$ and $h^{\prime}$ lies in $D\left(1+\bar{g}_{1}^{\prime}\right)$ then $\left(1+\bar{g}_{1}^{\prime}\right)\left(1+\bar{g}_{2}^{\prime}\right)=\left(1+\bar{g}_{1}^{\prime}\right)\left(1+\bar{h}^{\prime} \bar{g}_{2}^{\prime}\right)$.

(ii) If $\bar{g}_{1}^{\prime}+\bar{g}_{2}^{\prime} \neq 0$ in $R$ and $h^{\prime}$ lies in $D\left(\bar{g}_{1}^{\prime}+\bar{g}_{2}^{\prime}\right)$ then $\left(1+\bar{g}_{1}^{\prime}\right)\left(1+\bar{g}_{2}^{\prime}\right)=\left(1+\bar{h}^{\prime}\right)\left(1+\bar{g}_{1}^{\prime} \bar{g}_{2}^{\prime}\right)$.

Proof. (i ) By Lemma 2.1(i) $g_{2}^{\prime} h^{\prime}$ lies in $D\left(\bar{g}_{2}^{\prime}+\bar{g}_{2}^{\prime} \bar{g}_{1}^{\prime}\right)$. Since $R$ is strongly representational, $\bar{g}_{2}^{\prime} \bar{h}^{\prime}+\bar{g}_{1}^{\prime} \bar{g}_{2}^{\prime} \bar{h}^{\prime}=\bar{g}_{2}^{\prime}+\bar{g}_{2}^{\prime} \bar{g}_{1}^{\prime}$ which proves (i).

(ii) Since $R$ is strongly representational, $\bar{h}^{\prime}+\bar{h}^{\prime} \bar{g}_{1}^{\prime} \bar{g}_{2}^{\prime}=\bar{g}_{1}^{\prime}+\bar{g}_{2}^{\prime}$, 
hence $\left(1+\bar{g}_{1}^{\prime}\right)\left(1+\bar{g}_{2}^{\prime}\right)=\left(1+\bar{h}^{\prime}\right)\left(1+\bar{g}_{1}^{\prime} \bar{g}_{2}^{\prime}\right)$.

Proposition 4.4. (cf. [6, Prop. 2.2; 16, Prop. 1.5, p. 278]). Let $R$ be a strongly representational Witt ring for $G$. Let $P=$ $\Pi_{1}^{n}\left(1+\bar{g}_{i}^{\prime}\right)=1+P^{\prime}$, with $\operatorname{dim} P^{\prime}=2^{n}-1, n \geqq 1$, be a Pfister element of $R$ and let $h_{1}^{\prime}$ be in $D\left(P^{\prime}\right)$. Then there exist $h_{2}^{\prime}, \cdots, h_{n}^{\prime}$ in $G^{\prime}$ with $P=\prod_{1}^{n}\left(1+\bar{h}_{i}^{\prime}\right)$.

Proof. We use induction on $n$. If $n=1$ we have $P^{\prime}=\bar{g}_{1}^{\prime}$ and so $\bar{h}_{1}^{\prime}=\bar{g}_{1}^{\prime}$ and $1+\bar{g}_{1}^{\prime}=1+\bar{h}_{1}^{\prime}$. Hence we assume $n \geqq 2$ and that the proposition is true for $(n-1)$-fold Pfister elements. Let $Q=\Pi_{1}^{n-1}(1+$ $\left.\bar{g}_{i}^{\prime}\right)=1+Q^{\prime}$. Then $P=Q\left(1+\bar{g}_{n}^{\prime}\right)=Q+\bar{g}_{n}^{\prime} Q$. Hence $P^{\prime}=Q^{\prime}+\bar{g}_{n}^{\prime} Q$ and $2^{n}-1=\operatorname{dim} P^{\prime} \leqq \operatorname{dim} Q^{\prime}+\operatorname{dim} \bar{g}_{n}^{\prime} Q \leqq 2^{n-1}-1+2^{n-1}=2^{n}-1$, so that $\operatorname{dim} Q^{\prime}=2^{n-1}-1$ and $\operatorname{dim} \bar{g}_{n} Q=\operatorname{dim} Q=2^{n-1}$.

By hypothesis $h_{1}^{\prime}$ is in $D\left(P^{\prime}\right)=D\left(Q^{\prime}+\bar{g}_{n}^{\prime} Q\right)$. Since $R$ is representational there exist $x$ in $D\left(Q^{\prime}\right), y$ in $D(Q)$ such that $h_{1}^{\prime}$ is in $D\left(\bar{x}+\bar{g}_{n}^{\prime} \bar{y}\right)$. Since $Q=1+Q^{\prime}$ there exists an element $z$ in $D\left(Q^{\prime}\right)$ such that $y$ is in $D(1+\bar{z})$. By induction there exist $z_{2}, \cdots, z_{n-1}$ in $G^{\prime}$ with $Q=(1+\bar{z}) \Pi_{2}^{n-1}\left(1+\bar{z}_{i}\right)$. Now by Lemma 2.3 we have $1+\bar{z} \neq 0$ so that Lemma $4.3(\mathrm{i})$ shows that $P=Q\left(1+\bar{g}_{n}^{\prime}\right)=(1+\bar{z})$ $\left(1+\bar{g}_{n}^{\prime}\right) \prod_{2}^{n-1}\left(1+\bar{z}_{i}\right)=(1+\bar{z})\left(1+\bar{y} \bar{g}_{n}^{\prime}\right) \prod_{2}^{n-1}\left(1+\bar{z}_{i}\right)=\left(1+\bar{y} \bar{g}_{n}^{\prime}\right) Q$. Since $x$ lies in $D\left(Q^{\prime}\right)$ the induction hypothesis again yields $h_{2}^{\prime}, \cdots, h_{n-1}^{\prime}$ in $G^{\prime}$ with $Q=(1+\bar{x}) \prod_{2}^{n-1}\left(1+\bar{h}_{i}^{\prime}\right)$, so that $P=(1+\bar{x})\left(1+\bar{y} \bar{g}_{n}^{\prime}\right)$ $\Pi_{2}^{n-1}\left(1+\bar{h}_{i}^{\prime}\right)$. Again by Lemma $2.3, \bar{x}+\bar{y} \bar{g}_{n}^{\prime} \neq 0$ in $R$, so Lemma 4.3(ii) shows $(1+\bar{x})\left(1+\bar{y} \bar{g}_{n}^{\prime}\right)=\left(1+\bar{h}_{1}^{\prime}\right)\left(1+\overline{x y g_{n}^{\prime}}\right)$. Hence $P=\left(1+\bar{h}_{1}^{\prime}\right)$ $\left(1+\overline{x y g_{n}^{\prime}}\right) \prod_{2}^{n-1}\left(1+\bar{h}_{i}^{\prime}\right)$, proving Proposition 4.4.

COROLLARY 4.5. (cf. [6, Cor. 2.3] although our proof is different). Let $R$ be a strongly representational Witt ring for $G$ and $P=\prod_{1}^{n}\left(1+\bar{g}_{i}^{\prime}\right), g_{i}^{\prime}$ in $G^{\prime}$, an n-fold Pfister element of $R$. If $\operatorname{dim} P<2^{n}$ then $P=0$.

Proof. We again use induction on $n$. The corollary is clear if $n=1$ by [8, Rem. 1.24, Lem.]. Assume it is true for $(n-1)$-fold Pfister elements. Again let $Q=\Pi_{1}^{n-1}\left(1+\bar{g}_{i}^{\prime}\right)$ so that $P=Q+\bar{g}_{n}^{\prime} Q$ and $P^{\prime}=Q^{\prime}+\bar{g}_{n}^{\prime} Q$. If $\operatorname{dim} Q<2^{n-1}$ then $Q=0$ and so $P=0$ also. Hence we suppose $\prod_{1}^{n-1}\left(1+g_{i}^{\prime}\right)$ is anisotropic for $R$, i.e., $\operatorname{dim} Q=2^{n-1}$.

If $\operatorname{dim} P^{\prime}=2^{n}-1$ then by Lemma 1.4, the element -1 lies in $D\left(P^{\prime}\right)$ and so by Proposition 4.4 we see that $(\overline{1}+(-1))$ is a factor of $P$, whence $P=0$. Thus we may also suppose $\operatorname{dim} P^{\prime}<2^{n}-1$. By assumption, $\operatorname{dim} Q^{\prime}=2^{n-1}-1$ and $\operatorname{dim} \bar{g}_{n}^{\prime} Q=2^{n-1}$ but $\operatorname{dim} P^{\prime}=$ $\operatorname{dim}\left(Q^{\prime}+\bar{g}_{n}^{\prime} Q\right)<2^{n-1}-1+2^{n-1}=2^{n}-1$. Thus Proposition 2.4 shows the existence of $g^{\prime}$ in $G^{\prime}$ with $-g^{\prime}$ in $D\left(Q^{\prime}\right)$ and $g^{\prime}$ in $D\left(\bar{g}_{n}^{\prime} Q\right)=$ $g_{n}^{\prime} D(Q)$, by Lemma 2.1(i). Proposition 4.4 shows that then $Q=$ 
$\left(1-\bar{g}^{\prime}\right) Q_{1}$ and since $R$ is representational and $Q=1+Q^{\prime}$ there exists an $h^{\prime}$ in $D\left(Q^{\prime}\right)$ such that $g^{\prime}$ is in $D\left(\bar{g}_{n}^{\prime}+\bar{g}_{n}^{\prime} \bar{h}^{\prime}\right)$. Since $R$ is strongly representational this means $\bar{g}^{\prime}+\bar{g}^{\prime} \bar{h}^{\prime}=\bar{g}_{n}^{\prime}+\bar{g}_{n}^{\prime} \bar{h}^{\prime}$. Again by Proposition 4.4 , we may write $Q=\left(1+\bar{h}^{\prime}\right) S$. Hence $\bar{g}_{n}^{\prime} Q=\bar{g}^{\prime} Q$ and $P=Q+\bar{g}_{n}^{\prime} Q=\left(1+\bar{g}^{\prime}\right) Q=\left(1+\bar{g}^{\prime}\right)\left(1-\bar{g}^{\prime}\right) Q_{1}=0$, completing the proof.

CoRollary 4.6. (cf. [6, Cor. 2.4; 16, Cor. 1.7, p. 279]). Let $R$ be a strongly representational Witt ring for $G$. Then a nonzero $n$-fold Pfister element, $P$, of $R$ is round (Definition 2.14).

Proof. By Corollary 4.5 we have $\operatorname{dim} P=2^{n}$. Let $g^{\prime}$ lie in $D(P)=D\left(1+P^{\prime}\right)$. Since $R$ is strongly representational there exists an $h^{\prime}$ in $D\left(P^{\prime}\right)$ such that $g^{\prime}$ is in $D\left(1+\bar{h}^{\prime}\right)$ and $1+\bar{h}^{\prime}=\bar{g}^{\prime}+\bar{g}^{\prime} \bar{h}^{\prime}$ in $R$. By Proposition 4.4 we may write $P=\left(1+\bar{h}^{\prime}\right) Q$. Hence $\bar{g}^{\prime} P=$ $\left(\bar{g}^{\prime}+\bar{g}^{\prime} \bar{h}^{\prime}\right) Q=P$ and $P$ is round.

LEMMA 4.7. Let $R$ be a strongly representational Witt ring for $G$ and let $P$ be a Pfister element of $R$. Then for any $h^{\prime}$ in $D(P)$ and any $g^{\prime}$ in $G^{\prime}$ we have $P\left(1+\bar{g}^{\prime}\right)=P\left(1+\bar{h}^{\prime} \bar{g}^{\prime}\right)$.

Proof. By Corollary 4.6, $P$ is round so that $P \bar{h}^{\prime}=P$ and $P\left(1+\bar{g}^{\prime}\right)=P\left(1+\bar{h}^{\prime} \bar{g}^{\prime}\right)$.

Proposition 4.8. (cf. [6, Thm. 2.6; 16, Thm. 1.9, p. 281]). Let $R$ be a strongly representational Witt ring for $G$ with $g_{1}^{\prime}, \cdots, g_{n}^{\prime}$, $h_{1}^{\prime}, \cdots, h_{m}^{\prime}$ in $G^{\prime}, n \geqq 0, m \geqq 1$. If $P=\Pi_{1}^{n}\left(1+\bar{g}_{i}^{\prime}\right), Q=\Pi_{1}^{m}\left(1+\bar{h}_{i}^{\prime}\right)=$ $1+Q^{\prime}$ and $c_{1}$ lies in $D\left(P Q^{\prime}\right)$, then there exists $c_{2}, \cdots, c_{m}$ in $G^{\prime}$ such that $P Q=P \prod_{1}^{m}\left(1+\bar{c}_{i}\right)$. In particular, if -1 lies in $D\left(P Q^{\prime}\right)$ then $P Q=0$.

Proof. If $\operatorname{dim} P<2^{n}$, then by Corollary 4.5 we have $P=0$ and $D\left(P Q^{\prime}\right)=D(0)=\varnothing$. Thus we may assume $\operatorname{dim} P=2^{n}$. We now proceed by induction on $m$.

If $m=1$, then $c_{1}$ is in $D\left(P \bar{h}_{1}^{\prime}\right)=h_{1}^{\prime} D(P)$ by Lemma 2.1(i). Hence $c_{1}=h_{1}^{\prime} d_{1}$ with $d_{1}$ in $D(P)$. Then, by Lemma $4.7 P Q=P\left(1+\bar{c}_{1}\right)$.

We now assume $m>1$. By induction hypothesis we may assume the result for any element in $D\left(P S^{\prime}\right)$ with $S=\Pi_{1}^{m-1}\left(1+\bar{h}_{i}^{\prime}\right)=$ $1+S^{\prime}$. Now if $\operatorname{dim} P Q<2^{m+n}$, we have $P Q=0$ by Corollary 4.5 and for any $c_{1}$ in $G^{\prime} \supset D\left(P Q^{\prime}\right), P Q=0=P\left(1+\bar{c}_{1}\right) \Pi_{1}^{m-1}(\overline{1}+(-\overline{1}))$. Thus we also assume $\operatorname{dim}(P Q)=2^{n+m}$, and consequently, $\operatorname{dim}\left(P Q^{\prime}\right)=$ $2^{m+n}-2^{n}$. Now $Q=S\left(1+\bar{h}_{m}^{\prime}\right)=\bar{h}_{m}^{\prime} S+S$ so that $Q^{\prime}=\bar{h}_{m}^{\prime} S+S^{\prime}$ and $P Q^{\prime}=\bar{h}_{m}^{\prime} P S+P S^{\prime} . \quad$ Note $\operatorname{dim}\left(P Q^{\prime}\right)=2^{m+n}-2^{n}=2^{m+n-1}+\left(2^{m+n-1}-\right.$ $\left.2^{n}\right)=\operatorname{dim}\left(\bar{h}_{m}^{\prime} P S\right)+\operatorname{dim} P S^{\prime}$. Since $R$ is representational and $c_{1}$ is in 
$D\left(\bar{h}_{m}^{\prime} P S+P S^{\prime}\right)$ there exist $x$ in $D(P S)$ and $y$ in $D\left(P S^{\prime}\right)$ such that $c_{1}$ is in $D\left(\bar{h}_{m}^{\prime} \bar{x}+\bar{y}\right)$ and $\bar{h}_{m} \bar{x}+\bar{y} \neq 0$ by Lemma 2.3. Applying the induction hypothesis to $y$ in $D\left(P S^{\prime}\right)$ we find $c_{2}, \cdots, c_{m-1}$ in $G^{\prime}$ with $P S=P(1+\bar{y}) \Pi_{2}^{m-1}\left(1+\bar{c}_{i}\right)$. Now $P Q=P S\left(1+\bar{h}_{m}^{\prime}\right)=P S\left(1+\overline{x h_{m}^{\prime}}\right)$ by Lemma 4.7, so that $P Q=P(1+\bar{y})\left(1+\overline{x h_{m}^{\prime}}\right) \Pi_{2}^{m-1}\left(1+\bar{c}_{i}\right)$, which by Proposition 4.3 (ii) is $P\left(1+\bar{c}_{1}\right)\left(1+\overline{x y h_{m}^{\prime}}\right) \Pi_{2}^{m-1}\left(1+\bar{c}_{i}\right)$, the desired result.

Proposition 4.9. (cf. [6, Thm. 2.7]). Let $R$ be a strongly representational Witt ring for $G$ and $P=1+P^{\prime}, S, Q=1+Q^{\prime}$ respectively $n$-fold, $s$-fold and $r$-fold Pfister elements of $R$ with $s \geqq 0$, $r \geqq 1$ and $n \geqq r+s$. Suppose that there exists an element $q$ in $R$ with $P^{\prime}=Q^{\prime} S+q$ and $\operatorname{dim} q<2^{n}-2^{r+s}+2^{s}$. Then there exists an $(n-(r+s))$-fold Pfister element of $R$, denoted by $M$, with $P=S Q M$.

Proof. We first deal with the case $\operatorname{dim} P<2^{n}$, so that $P=0$, by Corollary 4.5. If $n>r+s$, then $P=S Q \Pi_{1}^{n-(r+s)}(\overline{1}+(-\overline{1}))=0$, which is the desired result. Thus we suppose $n=r+s$. If $\operatorname{dim} Q^{\prime} S<$ $2^{r+s}-2^{s}$, then $\operatorname{dim} Q S<2^{r+s}$ and $P=Q S=0$ by Corollary 4.5. If $\operatorname{dim} Q^{\prime} S=2^{r+s}-2^{s}$, then from $-\overline{1}=P^{\prime}=Q^{\prime} S+q$ we obtain $Q^{\prime} S=-\overline{1}-q$. Hence $2^{r+s}-2^{s}=\operatorname{dim} Q^{\prime} S \leqq \operatorname{dim}(-\overline{1})+\operatorname{dim} q<1+2^{s}$, which implies $r=1$ and $\operatorname{dim} Q^{\prime} S=2^{s}$. Then, by Definition $1.2,-1$ is in $D\left(Q^{\prime} S\right)$. Therefore, by Proposition 4.8 , we have $Q S=0=P$, which settles the case $\operatorname{dim} P<2^{n}$.

For the rest of the proof we may then assume $\operatorname{dim} P=2^{n}$. If $\operatorname{dim} Q^{\prime} S<2^{r+s}-2^{s}$ then $\operatorname{dim} Q^{\prime} S \leqq 2^{r+s}-2^{s}-2$ by [8, Rem. 1.24, Lem.]. Hence $2^{n}-1=\operatorname{dim} P^{\prime} \leqq \operatorname{dim} Q^{\prime} S+\operatorname{dim} q<2^{r+8}-2^{s}-2+$ $2^{n}-2^{r+s}+2^{s}=2^{n}-2$, which is impossible. Thus we may also assume $\operatorname{dim} Q^{\prime} S=2^{r+s}-2^{s}$ and $\operatorname{dim} q=2^{n}-2^{r+s}+2^{s}-1$. The rest of the proof is carried out by a double induction, first assuming $r=1$ and inducting on $s$, and then inducting on $r$.

Thus let $r=1, Q=(1+\bar{x}), x$ in $G^{\prime}$. If $s=0$, then $P^{\prime}=\bar{x}+q$ with $\operatorname{dim} q=2^{n}-2$. Hence $x$ lies in $D\left(P^{\prime}\right)$ and the conclusion follows from Proposition 4.4.

Next, let $s \geqq 1$ and write $S=S_{1}\left(1+\bar{g}^{\prime}\right), g^{\prime}$ in $G^{\prime}$ with $S_{1}=$ $1+S_{1}^{\prime} \neq 0$ an $(s-1)$-fold Pfister element. Then $P^{\prime}=\bar{x} S+q=$ $\bar{x} S_{1}+\left(\overline{x g^{\prime}} S_{1}+q\right)$ with $\operatorname{dim}\left(\overline{x g^{\prime}} S_{1}+q\right)<2^{s-1}+2^{n}-2^{1+s}+2^{s}=2^{n}-$ $2^{s}+2^{s-1}$. Thus by the induction hypothesis there exists an $(n-(1+$ $(s-1)))=(n-s)$-fold Pfister element $M=1+M^{\prime}$ with $P=$ $(1+\bar{x}) S_{1} M=(1+\bar{x}) S_{1} M^{\prime}+S_{1}+\bar{x} S_{1}$. Now since $\operatorname{dim} P=2^{n}$, we must have $\operatorname{dim}(1+\bar{x}) S_{1} M^{\prime}=2^{s}\left(2^{n-s}-1\right)=2^{n}-2^{s}$. Also, $P=\bar{x} S+$ $q+1$ with $\operatorname{dim} \bar{x} S=2^{s}$, and $\operatorname{dim} q=2^{n}-2^{s}-1$, so that $\operatorname{dim}(q+1)=$ $2^{n}-2^{s}$ and 1 lies in $D(q+1)$. Equating the two expressions for $P$, 
we get $P=(1+\bar{x}) S_{1} M^{\prime}+S_{1}+\bar{x} S_{1}=\bar{x} S+q+1=\bar{x} S_{1}+\overline{x g^{\prime}} S_{1}+q+1$, since $S=\left(1+\bar{g}^{\prime}\right) S_{1}$. Therefore $q+1$ lies in $R S_{1}$, where $S_{1}$ by Corollary 4.6, is round. Hence, by Proposition 2.16, there exists an element $p$ in $R$, with 1 in $D(p)$, such that $q+1=S_{1} p$ with $2^{n}-2^{s}=$ $\operatorname{dim}(q+1)=\operatorname{dim} S_{1} \cdot \operatorname{dim} p=2^{s-1} \operatorname{dim} p$. Hence $\operatorname{dim} p=2^{n-s+1}-2$. Since 1 lies in $D(p)$, there exists $p_{1}$ in $R$ such that $p=1+p_{1}$ with $\operatorname{dim} p_{1}<\operatorname{dim} p$ so that $\operatorname{dim} p_{1}=2^{n-s+1}-3$. Then $q+1=S_{1} p_{1}+S_{1}$ and $\operatorname{dim}(q+1)=2^{n}-2^{s} \leqq \operatorname{dim}\left(S_{1} p_{1}\right)+\operatorname{dim} S_{1} \leqq 2^{s-1}\left(2^{n-s+1}-3\right)+2^{s-1}=$ $2^{n}-2^{s}$ so that $\operatorname{dim} S_{1} p_{1}=2^{n}-3 \cdot 2^{s-1}$.

Substituting for $q+1$ we next find $P=(1+\bar{x}) S_{1} M^{\prime}+S_{1}+\bar{x} S_{1}=$ $\bar{x} S_{1}+\overline{x g^{\prime}} S_{1}+S_{1} p_{1}+S_{1}$ or $(1+\bar{x}) S_{1} M^{\prime}=\overline{x g^{\prime}} S_{1}+S_{1} p_{1}=\overline{x g^{\prime}}+\overline{x g^{\prime}} S_{1}^{\prime}+$ $S_{1} p_{1}$. Now $\left.\left.\operatorname{dim} \overline{\left(x g^{\prime}\right.} S_{1}^{\prime}+S_{1} p_{1}\right) \leqq \operatorname{dim} \overline{\left(x g^{\prime}\right.} S_{1}^{\prime}\right)+\operatorname{dim} S_{1} p_{1} \leqq 2^{s-1}-1+2^{n}-$ $3 \cdot 2^{s-1}=2^{n}-2^{s}-1<\operatorname{dim}(1+\bar{x}) S_{1} M^{\prime}$. Hence $x g^{\prime}$ lies in $D\left((1+\bar{x}) S_{1} M^{\prime}\right)$, so that by Proposition 4.8 there exists an $(n-s-1)$-fold Pfister element $N$ such that $P=(1+\bar{x}) S_{1} M=(1+\bar{x}) S_{1}\left(1+\overline{x g^{\prime}}\right) N$. Direct computation shows $(1+\bar{x})\left(1+\overline{x g^{\prime}}\right)=(1+\bar{x})\left(1+\bar{g}^{\prime}\right)$ so that finally $P=(1+\bar{x})\left(1+\bar{g}^{\prime}\right) S_{1} N=(1+\bar{x}) S N$, proving Proposition 4.9 for $r=1$ and all $s \geqq 0$.

Next suppose $r \geqq 2$ and assume, as our induction hypothesis that Proposition 4.9 is vaild for all $(r-1)$-fold Pfister elements and for all $s$-fold Pfister elements, $s \geqq 0$, satisfying the hypotheses. We write $Q=(1+\bar{y}) Q_{1}$ where $y$ is in $G^{\prime}$ and $Q_{1}=1+Q_{1}^{\prime}$ is an $(r-1)$ fold Pfister element. Then $Q^{\prime}=Q_{1}^{\prime}(1+\bar{y})+\bar{y}$ and by hypothesis $P^{\prime}=Q^{\prime} S+q=Q_{1}^{\prime}(1+\bar{y}) S+(\bar{y} S+q)$. Since $\operatorname{dim}(\bar{y} S+q) \leqq \operatorname{dim} \bar{y} S+$ $\operatorname{dim} q<2^{s}+2^{n}-2^{r+s}+2^{s}=2^{n}-2^{(r-1+s+1)}+2^{s+1}$ we may apply the induction hypothesis to the Pfister elements $P, Q_{1},(1+\bar{y}) S$, to obtain an $(n-(r+s))$-fold Pfister element $M$ such that $P=Q_{1}(1+\bar{y}) S M=$ $Q S M$, completing the proof.

CoRollary 4.10. (cf. [6, Rem. (1), p. 192]). Let $R$ be a strongly representational Witt ring for $G$ and $P=1+P^{\prime}$ an n-fold Pfister element of $R$. If there exist $g_{1}^{\prime}, g_{2}^{\prime}$ in $G^{\prime}$, and $q$ in $R$ such that $P^{\prime}=\bar{g}_{1}^{\prime}+\bar{g}_{2}^{\prime}+q$ with $\operatorname{dim} q<2^{n}-2$, then there exists an $(n-2)$ fold Pfister element $M$ in $R$ such that $P=\left(1+\bar{g}_{1}^{\prime}\right)\left(1+\bar{g}_{2}^{\prime}\right) M$.

Proof. $\quad P^{\prime}=\bar{g}_{1}^{\prime}\left(1+\bar{g}_{1}^{\prime} \bar{g}_{2}^{\prime}\right)+q=Q^{\prime} S+q$ with $Q=\left(1+\bar{g}_{1}^{\prime}\right), S=$ $\left(1+\bar{g}_{1}^{\prime} \bar{g}_{2}^{\prime}\right)$. Since $\operatorname{dim} q<2^{n}-2=2^{n}-2^{1+1}+2^{1}$, we must have $n \geqq 2$ and thus all the hypotheses of Proposition 4.9 are fulfilled with $r=s=1$, so that there is $(n-2)$-fold Pfister element $M$ in $R$ with $P=\left(1+\bar{g}_{1}^{\prime}\right)\left(1+\bar{g}_{1}^{\prime} \bar{g}_{2}^{\prime}\right) M=\left(1+\bar{g}_{1}^{\prime}\right)\left(1+\bar{g}_{2}^{\prime}\right) M$.

CoRollary 4.11. (cf. [6, Rem. (2), p. 192]). Let $R$ be a strongly representational Witt ring for $G$ and $P$ and $S$ respectively 
$n$-fold and s-fold Pfister elements of $R$. If there exists an element $q$ in $R$ with $P^{\prime}=P-1=S+q$ with $\operatorname{dim} q<2^{n}-2^{s}$, then $P=2 S M$ for some $(n-s-1)$-fold Pfister element $M$ in $R$.

Proof. Proposition 4.9 is applicable with $Q=(\overline{1}+\overline{1})=2$.

Corollary 4.12. (cf. [6, Rem. (3), p. 192]). Let $R$ be a strongly representational Witt ring for $G$ and $P$ and $Q$ respectively $n$-fold and $r$-fold Pfister elements of $R, n \geqq r \geqq 1$. If there exists an element $q$ in $R$ with $P=Q+q$ with $\operatorname{dim} q<2^{n}-2^{r}+1$, then there exists an $(n-r)$-fold Pfister element $M$ with $P=Q M$.

Proof. Since $P=Q+q$, we have $P^{\prime}=Q^{\prime}+q$. Proposition 4.9 with $s=0, S=\overline{1}$, then yields the result.

The proofs of the following results will be omitted since they are obtained by subjecting the proofs in [6] and [7] to changes similar to those made in proving Propositions 4.3-4.9.

Proposition 4.13. (cf. [7, Thm. 2.1]). Let $R$ be a strongly representational Witt ring for $G$ and $P, Q$ respectively $n$-fold and $r$-fold Pfister elements in $R$ with $n \geqq r$. Then the following are equivalent:

(i) There exists an $(n-r)$-fold Pfister element $M$ in $R$ with $P=Q M$.

(ii) $P$ lies in $Q I^{(n-r)}$, where $I^{(n-r)}$ is the ideal of $R$ generated by all $(n-r)$-fold Pfister elements.

(iii) $P$ lies in $R Q$, with $P \neq 0$ if $r=n$.

Definition 4.14. (cf. [6, Def. 4.1]). Let $P_{1}, \cdots, P_{m}$ be $n$-fold Pfister elements in a Witt ring $R$ for $G$. These are said to be $r$-linked if there exists an $r$-fold Pfister element $Q$ in $R$ and $(n-r)$ fold Pfister elements $M_{1}, \cdots, M_{m}$ such that $P_{i}=Q M_{i}, i=1, \cdots, m$. The natural number $r$ is called the linkage number if $P_{1}, \cdots, P_{m}$ are $r$-linked but not $(r+1)$-linked. If the linkage number is $\geqq n-1$, then $P_{1}, \cdots, P_{m}$ are called linked.

Proposition 4.15. (cf. [6, Prop. 4.4]). Let $R$ be a strongly representational Witt ring. Two $n$-fold Pfister elements $P_{1}, P_{2}$ in $R$ are $r$-linked if and only if $\operatorname{dim}\left(P_{1}-P_{2}\right) \leqq 2^{n+1}-2^{r+1}$, equality occuring if $r$ is the linkage number. In particular, if $\operatorname{dim}\left(P_{1}-P_{2}\right) \leqq 2^{n}$ then $P_{1}$ and $P_{2}$ are linked.

Proposition 4.16. (cf. [6, Thm. 4.5]). Let $R$ be a strongly representational Witt ring for $G ; P, Q$ nonzero $n$-fold Pfister 
elements in $R$ and $g^{\prime}, h^{\prime}$ elements of $G^{\prime} . \quad$ Then $\operatorname{dim}\left(\bar{g}^{\prime} P+\bar{h}^{\prime} Q\right)=2^{n+1}$ or $2^{n+1}-2^{r+1}$, where $r>0$ is the linkage number of $P$ and $Q$.

REMARK 4.17. The proof of [4, Satz 16] can also be extended to torsion free representational Witt rings keeping in mind that such a ring is, by Corollary 2.21, dimensional, so that for any element $r$ in it we have $\operatorname{dim}(m r)=m \operatorname{dim} r$ and $D(m r)=D(r)$, where $m$ is any natural number. This yields

Proposition 4.18. Let $R$ be a reduced representational Witt ring for $G$. Let $r$ be a round element of $R$ with $\operatorname{dim} r=n$. If $n=2^{t} u$ with $(2, u)=1$, there exists a unique Pfister element $P$ in $R$ with $r=u P$.

REMARK. The hypotheses of Proposition 4.18 are fulfilled in case $R=S / I(Y)$ with $S$ a representational Witt ring for $G$ and $Y$ a saturated subset of $X(S)$ by Proposition 2.24. Since, as we shall show in Proposition 6.7, the ring $W(F)$ is representational for $F$ a field of characteristic $\neq 2$ and the rings $W_{T}$ of [4] are of the form $W(F) / I(Y)([8, \S 2])$, Proposition 4.18 does yield [4, Satz 16].

5. Remarks on [3]. In this section we show that [3, Prop. 5.1] is valid for dimensional Witt rings and that by [17] some of the results of $[3,5]$ also carry over to the case of representational Witt rings. We have preferred, for the readers sake, to give fairly complete proofs of Theorems 5.4 and 5.8, but wish to emphasize here that the main ideas of the proofs come from [3].

Lemma 5.1. Let $R$ be a reduced Witt ring for $G$ and $\sum_{1}^{n} \bar{g}_{i}^{\prime}=$ $\sum_{1}^{m} \bar{h}_{j}^{\prime}$ an element of $R$. Then $(-1)^{(n(n-1)) / 2} \Pi_{1}^{n} \bar{g}_{i}^{\prime}=(-1)^{(m(m-1)) / 2} \Pi_{1}^{m} \bar{h}_{j}^{\prime}$ in $R$.

Proof. Since $R$ is reduced, by Remark 1.6 two elements $x$ and $y$ of $R$ are equal if and only if $\sigma(x)=\sigma(y)$ for all $\sigma$ in $X(R)$. Now for a fixed signature $\sigma$, let $p$ of the $\sigma\left(\bar{g}_{i}^{\prime}\right)$ and $p^{\prime}$ of the $\sigma\left(\bar{h}_{j}^{\prime}\right)$ be 1 , so that $n-p$ of the $\sigma\left(\bar{g}_{i}^{\prime}\right)$ and $m-p^{\prime}$ of the $\sigma\left(\bar{h}_{j}^{\prime}\right)$ are -1 . Then

$$
p-(n-p)=2 p-n=p^{\prime}-\left(m-p^{\prime}\right)=2 p^{\prime}-m .
$$

Thus

$$
\sigma\left[(-1)^{(n(n-1)) / 2} \prod_{1}^{n} \bar{g}_{i}^{\prime}\right]=(-1)^{(n(n-1)) / 2+n-p}=(-1)^{\left.\left(n^{2}+n-2 p\right)\right) / 2} .
$$

But $\left(n^{2}+n-2 p\right) / 2=\left(n^{2}+n-2 p-4 p n+4 p^{2}\right) / 2=\left((n-2 p)^{2}+(n-2 p) / 2 \bmod 2\right.$. Hence $\sigma\left[(-1)^{(n(n-1)) / 2} \Pi_{1}^{n} \bar{g}_{i}^{\prime}\right]=(-1)^{\left((n-2 p)^{2}(n-2 p) / 2\right.}=(-1)^{\left(\left(m-2 p^{\prime}\right)^{2}+\left(m-2 p^{\prime}\right) / 2\right.}=$ 
$\sigma\left[(-1)^{(m(m-1)) / 2} \mathrm{Il}_{1}^{m} \bar{h}_{j}^{\prime}\right]$, proving Lemma 5.1.

Lemma 5.2. Let $R$ be a Witt ring for $G$ and $Y$ a set of signatures of $R$ such that $\bar{R}=R / I(Y)$ is dimensional. Let $r=\sum_{1}^{n} \bar{g}_{2}^{\prime}$ and $\widetilde{r}$ be elements of $R$ with $r \equiv 2 \widetilde{r} \bmod I(Y)$ and let the image of $r$ in $\bar{R}$ be denotes by $\bar{r}$. Then if $\operatorname{dim}_{\bar{l}} \bar{r}=n$, the element $-g_{1}^{\prime}+$ $\sum_{2}^{n} g_{i}^{\prime}$ of $Z[G]$ is isotropic for $\bar{R}$.

Proof. By [14, Prop. 3.14], $\bar{R}$ is also a Witt ring for $G$. Since $\operatorname{dim}_{\bar{i}} \bar{r}=n$, Definition 1.2 shows that $g_{1}^{\prime}$ lies in $D_{\bar{R}}(\bar{r})$. In $\bar{R}$ we have $\bar{r}=2 \overline{\widetilde{r}}$ so that $g_{1}^{\prime}$ also lies in $D_{\bar{R}}(2 \widetilde{r})=D_{\bar{l}}(\bar{r})[8$, Def. 1.18 and Thm. 1.17(iii)]. Hence $\widetilde{\widetilde{r}}=\overline{\bar{g}}_{1}^{\prime}+x$, where $x$ is an element of $\bar{R}$ with $\operatorname{dim}_{\bar{R}} x<\operatorname{dim}_{\bar{l}} \overline{\widetilde{r}} . \quad$ But $n=\operatorname{dim}_{\bar{l}} \bar{r}=\operatorname{dim}_{\overline{l i}} 2 \overline{\widetilde{r}}=2 \operatorname{dim}_{\bar{l}} \overline{\widetilde{r}}$ [8, Thm. 1.17(i)]. Therefore $\operatorname{dim}_{\bar{\pi}} x<n / 2$. Thus in $\bar{R}$ we have $\sum_{1}^{n} \overline{\bar{g}}_{i}^{\prime}=\overline{\bar{g}}_{1}^{\prime}+\overline{\bar{g}}_{1}^{\prime}+2 x$ with $\operatorname{dim}_{\bar{\pi}} 2 x=2 \operatorname{dim}_{\bar{R}} x<n$. Then $-\ddot{\bar{g}}_{1}^{\prime}+\sum_{2}^{n} \overline{\bar{g}}_{i}^{\prime}=2 x$ in $\bar{R}$, so that by Definition 1.3, the lemma follows.

Lemma 5.3. Let $R$ be a Witt ring for $G$ and $Y \supset Y_{j}, j=1,2$, sets of signatures of $R$. Let $r=\sum_{1}^{n} \bar{g}_{i}^{\prime}$ be an element of $R / I(Y)$ with $n$ even such that $\Pi_{1}^{n} \bar{g}_{i}^{\prime}=(-1)^{n / 2}$ in $R / I(Y)$. Assume there exist elements $r_{Y_{j}}$ in $R / I\left(Y_{j}\right)$ with $\bar{r}=2 r_{Y_{j}}$ in $R / I\left(Y_{j}\right)$ where $\bar{r}$ denotes both images of $r$ in $R / I\left(Y_{j}\right)$. If $\operatorname{dim}_{R / I\left(Y_{1}\right)} r_{Y_{1}}=(n-2) / 2$ and $R / I\left(Y_{2}\right)$ is dimensional then $\sum_{1}^{n} g_{i}^{\prime}$ is isotropic for $R / I\left(Y_{2}\right)$.

Proof. In $R / I\left(Y_{1}\right)$ we may write $\sum_{1}^{n} \overline{\bar{g}}_{i}^{\prime}=2 \sum_{1}^{(n-2) / 2} \overline{\bar{h}}_{i}^{\prime}$ for $h_{i}^{\prime}$ in $G^{\prime}$. Since there is a natural homomorphism $R / I(Y) \rightarrow R / I\left(Y_{1}\right)$, Lemma 5.1 shows that $(-1)^{(n(n-1)+n\rangle / 2}=(-1)^{((n-2)(n-3)) / 2}$ in $R / I\left(Y_{1}\right)$. Straightforward computation yields $(-1)^{n / 2}=-1$ in $R / I\left(Y_{1}\right)$. Since $R / I\left(Y_{1}\right)$ admits homomorphisms to $Z$, we have $-1 \neq 1$ in $R / I\left(Y_{1}\right)$ so that $n / 2$ is odd. If $\sum_{1}^{n} g_{i}^{\prime}$ were anisotropic for $R / I\left(Y_{2}\right)$, then $\operatorname{dim}_{R / I\left(Y_{2}\right)} 2 r_{Y_{2}}=n$ and so by [8, Thm. 1.17(i)] $\operatorname{dim}_{R / I\left(Y_{2}\right)} r_{Y_{2}}=n / 2$. But then again by Lemma 5.1 and using the homomorphism $R / I(Y) \rightarrow$ $R / I\left(Y_{2}\right)$, we have $(-1)^{(n(n-1)) / 2}(-1)^{n / 2}=(-1)^{(n(n-1)) / 2}$ in $R / I\left(Y_{2}\right)$ so that $(-1)^{n / 2}=1$ in $R / I\left(Y_{2}\right)$. Just as above $-1 \neq 1$ in $R / I\left(Y_{2}\right)$. This contradiction proves Lemma 5.3.

Theorem 5.4. (cf. [3, Prop. 5.1]). Let $R$ be a Witt ring for $G, Y$ a closed set of signatures of $R$ and $\mathfrak{F}$ a family of subsets of $Y$ such that an element of $Z[G]$ is isotropic for $R / I(Y)$ if and only if it is isotropic for $R / I\left(Y^{\prime}\right)$ for all $Y^{\prime}$ in $\widetilde{F}$. Suppose further that for all $Y^{\prime}$ in $\widetilde{\Im}$, the ring $R / I\left(Y^{\prime}\right)$ is dimensional. Let $C(Y, \boldsymbol{Z})$ be the ring of continuous functions from $Y$ (with the Zariski topology) to $Z$ (with the discrete topology), and let $f$ be an element of $\boldsymbol{Z} \cdot 1+C(Y, 2 \boldsymbol{Z})$. If for all $Y^{\prime}$ in $\widetilde{\wp}$ there exist elements 
$r_{Y^{\prime}}$ in $R$ such that the restriction of $f$ to $Y^{\prime}$ is given by $f(\sigma)=\sigma\left(r_{Y^{\prime}}\right)$ for all $\sigma$ in $Y^{\prime}$, there exists an element $r$ in $R$ with $f(\sigma)=\sigma(r)$ for all $\sigma$ in $Y$.

Proof. Since $Y$ is closed it is clear that $X(R / I(Y))=Y$. By [15, Thm. 3.18(i)] for any $f$ in $C(Y, Z)$ there exists an element $r_{0}$ of $R / I(Y)$ and a natural number $m$ such that $\left(2^{m} f\right)(\sigma)=\sigma\left(r_{0}\right)$ for all $\sigma$ in $Y$. It clearly suffices to treat the case $m=1$, for once this is done, Theorem 5.4 is proved for $2^{m-1} f$, which then yields the result for $2^{m-2} f$, etc.

Thus for $f$ in $Z \cdot 1+C(Y, 2 Z)$ we may suppose $2 f(\sigma)=\sigma\left(r_{0}\right)$ for all $\sigma$ in $Y$ and some element $r_{0}$ in $R / I(Y)$. Hence for all $\sigma$ in $Y^{\prime}$, with $Y^{\prime}$ in $\widetilde{\mho}$, we have $\sigma\left(r_{0}\right)=2 \sigma\left(r_{Y^{\prime}}\right)$, so that $\bar{r}_{0}=2 \bar{r}_{Y^{\prime}}$ in $R / I\left(Y^{\prime}\right)$ by Remark 1.6. Let $\operatorname{dim}_{R / I(Y)} r_{0}=n$ and $r_{0}=\sum_{1}^{n} \bar{g}_{i}^{\prime}$. Since Theorem 5.4 is obviously true for constant functions, we may assume without loss of generality that $f(Y) \subseteq 2 Z$. Thus for all $\sigma$ in $Y$, we have $\sigma\left(r_{0}\right) \equiv O(4)$. For a fixed $\sigma$ in $Y$, let $\sigma\left(\bar{g}_{i}^{\prime}\right), i=1, \cdots, n$, be $1 p$ times so that $\Pi_{1}^{n} \sigma\left(\bar{g}_{i}^{\prime}\right)=(-1)^{n-p}$ in $R / I(Y)$. Then $2 p-n \equiv O$ (4) so that $n$ is even and $p \equiv n / 2(2)$. Thus $\Pi_{1}^{n} \sigma\left(\bar{g}_{i}^{\prime}\right)=(-1)^{n / 2}$ or, by Remark 1.6, $\Pi_{1}^{n} \bar{g}_{i}^{\prime}=(-1)^{n / 2}$ in $R / I(Y)$.

If for some $Y^{\prime}$ in $\widetilde{F}$ we had $\operatorname{dim}_{R / I\left(Y^{\prime}\right)} \bar{r}_{Y^{\prime}}=(n-2) / 2$, Lemma 5.3, shows that $\sum_{1}^{n} g_{i}^{\prime}$ would be isotropic for all $R / I\left(Y^{\prime \prime}\right)$ with $Y^{\prime \prime}$ in $\mathfrak{F}$, which by the hypothesis on $\mathfrak{F}$ would make $\sum_{1}^{n} g_{i}^{\prime}$ isotropic for $R / I(Y)$, a contradiction. Hence for all $Y^{\prime}$ in $\widetilde{F}$ we know $\operatorname{dim}_{R / I\left(Y^{\prime}\right)} \bar{r}_{Y^{\prime}}$ $\neq(n-2) / 2$.

If $\sum_{1}^{n} g_{i}^{\prime}$ is anisotropic for $R / I\left(Y^{\prime}\right)$, with $Y^{\prime}$ in $\widetilde{F}$, Lemma 5.2 shows that $-g_{1}^{\prime}+\sum_{2}^{n} g_{i}^{\prime}$ is isotropic for $R / I\left(Y^{\prime}\right)$. If $\sum_{1}^{n} g_{i}^{\prime}$ is isotropic for $R / I\left(Y^{\prime}\right)$ with $Y^{\prime}$ in $\widetilde{\mho}$, then by [8, Rem. 1.24, Lem.] $\operatorname{dim}_{R / I\left(Y^{\prime}\right)} \bar{r}_{0} \leqq n-2$. Since $\operatorname{dim}_{R / I\left(Y^{\prime}\right)} \bar{r}_{0}=2 \operatorname{dim}_{R / I\left(Y^{\prime}\right)} \bar{r}_{Y^{\prime}}$ [8, Thm. 1.17 (i)], this together with the last paragraph shows $\operatorname{dim}_{R / I\left(Y^{\prime}\right)} \bar{r}_{Y^{\prime}}<$ $(n-2) / 2$. Now, in $R / I\left(Y^{\prime}\right)$, we have $-\overline{\bar{g}}_{1}^{\prime}+\sum_{2}^{n} \overline{\bar{g}}_{i}^{\prime}=2 \bar{r}_{Y^{\prime}}-\overline{\bar{g}}_{1}^{\prime}-\overline{\bar{g}}_{1}^{\prime}$. Since, in $R / I\left(Y^{\prime}\right)$, the element $2 \bar{r}_{Y^{\prime}}$ is the sum of fewer than $n-2$ images of elements of $G^{\prime}$, the element $-g_{1}^{\prime}+\sum_{2}^{n} g_{i}^{\prime}$ is again isotropic for $R / I\left(Y^{\prime}\right)$. Hence $-g_{1}^{\prime}+\sum_{2}^{n} g_{2}^{\prime}$ is isotropic for $R / I(Y)$.

Now setting $r_{1}=r_{0}-\bar{g}_{1}^{\prime}-\bar{g}_{1}^{\prime}$, we see that $\operatorname{dim}_{R / I(X)} r_{1}<$ $\operatorname{dim}_{R / I(Y)} r_{0}$. Let $f_{1}$ in $C(Y, Z)$ be defined by $f_{1}(\sigma)=f(\sigma)-\sigma\left(\bar{g}_{1}^{\prime}\right)$ for all $\sigma$ in $Y$. Then $2 f_{1}(\sigma)=\sigma\left(r_{1}\right)$ and for all $\sigma$ in $Y^{\prime}$, we have $f_{1}(\sigma)=$ $\sigma\left(r_{Y^{\prime}}-\bar{g}_{1}^{\prime}\right)$; thus the proof is completed by induction on $n=$ $\operatorname{dim}_{R / I(Y)} r_{0}$, since if $n=2$ the above proof shows $r_{0}=\bar{g}_{1}^{\prime}+\bar{g}_{1}^{\prime}$ and $f(\sigma)=\sigma\left(\bar{g}_{1}^{\prime}\right)$.

Remark. By [8, Cor. 2.12], Theorem 5.4 applies to $R=W(C)$, the Witt ring of classes of nondegenerate symmetric bilinear $C$ forms when $C$ is a connected semilocal ring all of whose residue 
class fields contain at least 3 elements if $Y$ and the elements of $\mathfrak{F}$ are saturated. Since [8, Thm. 2.11] shows that the notion of isotropic used here and that used in [3] coincide and [8, Thm. 2.15] shows that the Witt rings of [3] are of the form $W(C) / I(Y)$ with $Y$ saturated, Theorem 5.4 really does yield [3, Prop. 5.1] in case $C$ is a field of characteristic $\neq 2$.

The interest in Theorem 5.4 lies in the light it sheds on the image of $R / I(Y)$ in $C(Y, Z)$ since by [15, Thm. 3.18(iv)] this image is always contained in $Z \cdot 1+C(Y, 2 Z)$. In [3, 33 and Cor. 5.2] it is shown that in case $R=W(C)$, with $C$ a field of characteristic $\neq 2$, the family $\widetilde{\wp}$ may be taken to be the family of finite saturated subsets of $Y$. Thus in the framework of [3] the description of $\operatorname{Im}(R / I(Y))$ in $C(Y, Z)$ is reduced, as we shall show below, to the case of Witt rings for finite groups. Unfortunately we are unable at this time to prove an analogue of [3, Cor. 5.2] for representational Witt rings for $G$. Nevertheless, as we now point out, part of [3, Thm. 5.3] does carry over to the abstract situation.

Lemma 5.5. Let $R$ be a Witt ring for $G$ and $Y \neq \varnothing$ a subset of $X(R)$. Then $Y$ is finite if and only if $G^{\prime} / \Gamma(Y)$ is finite so that $R / I(Y)$ is a Witt ring for the finite group $G^{\prime} / \Gamma(Y)$.

Proof. If $Y=\left\{\sigma_{1}, \cdots, \sigma_{n}\right\}$ then the sequence

$$
1 \longrightarrow \Gamma(Y)=\bigcap_{1}^{n} \Gamma\left(\sigma_{\imath}\right) \longrightarrow G^{\prime} \longrightarrow \prod_{1}^{n}\{ \pm 1\}
$$

is exact, so that $G^{\prime} / \Gamma(Y)$ is finite. Conversely, if $G^{\prime} / \Gamma(Y)$ is finite, since $Y$ may be identified with the characters it induces on $G^{\prime} / \Gamma(Y)$, it is bijective with a subset of the character group of a finite abelian group and so is finite.

Definition 5.6. Let $R$ be a Witt ring for $G$ and $Y$ a subset of $X(R)$. Then $Y$ is a fan [4, Satz 20(ii)] if every character of $G^{\prime} / \Gamma(Y)$ that maps $-\Gamma(Y)$ to -1 induces a signature of $Y$.

Lemma 5.7. Let $Y$ be a fan and let $g_{0}^{\prime}$ be an element in $G^{\prime}$ with $g_{0}^{\prime} \Gamma(Y) \neq-\Gamma(Y)$. Then $Y^{\prime}=\left\{\sigma\right.$ in $\left.Y \mid \sigma\left(\bar{g}_{0}^{\prime}\right)=1\right\}$ is also a fan.

Proof. Any character of $G^{\prime} / \Gamma\left(Y^{\prime}\right)$ induces a character of $G^{\prime} / \Gamma(Y)$ since $\Gamma\left(Y^{\prime}\right) \supset \Gamma(Y)$. Thus any character of $G^{\prime} / \Gamma\left(Y^{\prime}\right)$ sending $-\Gamma\left(Y^{\prime}\right)$ to -1 induces a signature of $Y$ which sends $\bar{g}_{0}^{\prime}$ to 1 , i.e., a signature of $Y^{\prime}$, so that $Y^{\prime}$ is, indeed, a fan.

TheOREM 5.8. (cf. [3, Thm. 5.3(b), (c)]). Let $R$ be a Witt ring for $G$ and $Y \subset X(R)$. For any finite set $P$ denote by $|P|$ the card- 
inality of $P$. Then the following are equivalent:

(i) For each finite fan $Y^{\prime} \subset Y$ we have $\sum_{\sigma i n Y^{\prime}} f(\sigma) \equiv 0 \bmod \left|Y^{\prime}\right|$.

(ii) Let $f$ be in $C(Y, Z)$. For every finite fan $Y^{\prime} \subset Y$ there is an element $r_{Y^{\prime}}$ in $R$ with $f(\sigma)=\sigma\left(r_{Y^{\prime}}\right)$ for all $\sigma$ in $Y^{\prime}$.

Proof. (i ) $\Rightarrow$ (ii). Let $Y^{\prime}$ be a finite fan and $f$ an element of $C(Y, Z)$. We identify $Y^{\prime}$ with all the characters of $G^{\prime} / \Gamma\left(Y^{\prime}\right)$ sending $-\Gamma\left(Y^{\prime}\right)$ to -1 . Now since every element of $G^{\prime} / \Gamma\left(Y^{\prime}\right)$ has order 2 and $G^{\prime} \mid \Gamma\left(Y^{\prime}\right)$ is finite by Lemma 5.5, we may write $G^{\prime} / \Gamma\left(Y^{\prime}\right)=$ $\left\{\Gamma\left(Y^{\prime}\right),-\Gamma\left(Y^{\prime}\right)\right\} \times H$ and $Y^{\prime}$ is then identified with $\hat{H}$, the character group of $H$. Now we also write $f$ for the function from $\hat{H}$ to $Z$ induced by $f$ and define functions $f_{h}: \hat{H} \rightarrow \boldsymbol{Z}$ by $f_{h}(\chi)=\chi(h)$ for each $h$ in $H$. Then if we set $m_{f, h}=(1 /|H|) \sum_{\text {Xin } \hat{H}} f(\chi) \chi(h)$ it is an immediate consequence of the orthogonality relation (10) on p. 181 of [21] that $f=\sum_{h} m_{f, h} f_{h}$. By (i) $m_{f, 1}$ lies in $Z$.

Next, we show that $m_{f, h}$ is in $Z$ for all $h$ in $H$. Let $S(h)$ denote the characters in $\hat{H}$ for which $\chi(h)=1$. We may then write $H=\{1, h\} \times H_{1}$ and may identify $S(h)$ with $\hat{H}_{1}$. Thus $|S(h)|=\left|H_{1}\right|=$ $(|H| / 2)$ and by Lemma $5.7 S(h)=\hat{H}_{1}$ is the subfan $Y^{\prime \prime}$ of $Y^{\prime}$ consisting of all singatures sending $g_{0}^{\prime} \Gamma\left(Y^{\prime}\right)=h$ to 1 . Now $m_{f, h}=$ $(1 /|H|)\left[\sum_{\chi \text { ins }(h)} f(\chi)-\sum_{\chi \text { not in } S(h)} f(\chi)\right]=(2 /|H|) \sum_{x \text { ins }(h)} f(\chi)-(1 /|H|)$ $\sum_{\chi \text { in } \hat{H}} f(\chi)$. Applying (i) to the fan $Y^{\prime \prime}$ shows that first term is in $\boldsymbol{Z}$ and since the second term is $m_{f, 1}$ we have shown that $m_{f, h}$ lies in $Z$.

Finally, let $r_{Y^{\prime}}=\sum_{h \text { in } H} m_{f, h} \bar{g}^{\prime}$ where $g^{\prime} \Gamma\left(Y^{\prime}\right)=h$. Then for all $\sigma$ in $Y^{\prime}$, we see $\sigma\left(r_{Y^{\prime}}\right)=\sum_{h \text { in } H} m_{f, h} \sigma\left(\bar{g}^{\prime}\right)=\sum_{h \text { in } H} m_{f, h} \chi(h)=$ $\left(\sum_{h \text { in } H} m_{f, h} f_{h}\right)(\chi)=f(\chi)=f(\sigma)$, proving (ii).

(ii) $\Rightarrow$ (i ) Suppose first that for all $\sigma$ in $Y^{\prime}$ there exists $g^{\prime}$ in $G^{\prime}$ with $f(\sigma)=\sigma\left(\bar{g}^{\prime}\right)$. If $g^{\prime}$ lies in $\Gamma\left(Y^{\prime}\right) \cup-\Gamma\left(Y^{\prime}\right)$, then $\sum_{o \text { in } Y^{\prime}} f(\sigma)=$ $\pm\left|Y^{\prime}\right|$, proving (i) in this case.

If $g^{\prime} \Gamma\left(Y^{\prime}\right) \neq \Gamma\left(Y^{\prime}\right)$ or $-\Gamma\left(Y^{\prime}\right)$ then we again write $G^{\prime} / \Gamma\left(Y^{\prime}\right)=$ $\left\{\Gamma\left(Y^{\prime}\right),-\Gamma\left(Y^{\prime}\right)\right\} \times H$ with $g^{\prime} \Gamma\left(Y^{\prime}\right) \neq 1$ in $H$, and identify $Y^{\prime}$ with $\hat{H}$, the character group of $H$. Then $\sum_{\sigma i n Y^{\prime}} f(\sigma)=\sum_{\chi \text { in } \hat{H}} \chi(h)$ for $h=g^{\prime} \Gamma\left(Y^{\prime}\right) \neq 1$ in $H$. By (8) on p. 181 of [21] this sum is $O$ and so (i) is also valid in this case.

For an arbitrary $f$, by (ii), there exists an $r_{Y^{\prime}}$ in $R$ so that for all $\sigma$ in $Y^{\prime}$ we have $f(\sigma)=\sigma\left(r_{Y^{\prime}}\right)$. Let $r_{Y^{\prime}}=\sum_{1}^{n} \bar{g}_{i}^{\prime}$. Then $f(\sigma)=$ $\sum_{1}^{n} \sigma\left(\bar{g}_{i}^{\prime}\right)$ and $\sum_{\sigma \mathrm{in} Y^{\prime}} f(\sigma)=\sum_{i=1}^{n} \sum_{\sigma_{\mathrm{in} Y^{\prime}}} \sigma\left(\bar{g}_{i}^{\prime}\right) \equiv O \bmod \left|Y^{\prime}\right|$ by what was proved above.

Next we point out that by using the main result of [17] the remainder of [3, Thm. 5.3] is valid for representational Witt rings for finite groups:

By Remark 2.30, if $R$ is a reduced representational Witt ring for a finite group $G$, then $\left(X, G^{\prime} / \Gamma(X(R))\right)$ is a finite space of order- 
ings as defined in [17 and 18]. By [17, Thm. 4.11 and 18, Rem. 1.8], there exists a pythagorean field $F$ with $X \cong$ the space of orderings of $F, G^{\prime} / \Gamma(X(R)) \cong \dot{F} / \dot{F}^{2}$ and $R \cong W(F)$. Hence [3, Thm. $5.3(\mathrm{~b}) \Rightarrow(\mathrm{a})]$ yields the following:

THEOREM 5.9. Let $R$ be a representational reduced Witt ring for a finite group $G$ with $\Gamma(X(R))=1$. Then if for $f$ in $C(X(R)$, $Z)$ there exists $r_{Y}$ for each fan $Y \subset X(R)$ with $f(\sigma)=\sigma\left(r_{Y}\right)$ for all $\sigma$ in $Y$, there exists $r$ in $R$ with $f(\sigma)=\sigma(r)$ for all $\sigma$ in $X(R)$.

REMARK. It is possible, by using [17], to prove Theorem 5.9 without any reference to fields and valuations. Essentially the analogues of the results of $[3, \S 4]$ hold for reduced representational Witt rings for finite $G$ whose space of signatures is connected in the sense of [17]. On the basis of this, the proof in [3] can be adapted to the abstract situation.

6. Witt rings of semilocal rings. In this Section we prove results about Witt rings of bilinear forms over semilocal rings that enable us to apply the results of the previous sections. Throughout the rest of this paper $C$ will denote a commutative connected semilocal ring and $U(C)$ its group of units. By a space over $C$ we shall mean a pair $(E, B)$ where $E$ is a finitely generated projective (whence free) $C$-module and $B$ is a symmetric nondegenerate bilinear form on $E$. Isometries will be written as $\cong$ and for any natural number $m$, the space $E \perp \cdots \perp E$ ( $m$ times) will be denoted by $m E$. An element $e$ of $E$ is called primitive if it can be augmented to a basis of $E$. A space $(E, B)$ is isotropic if there is a primitive element $e$ in $E$ with $B(e, e)=0$, and weakly isotropic if for some natural number $m$, the space $m E$ is isotropic. The space $C e_{1} \perp \cdots \perp$ $C e_{n}$ with $B\left(e_{i}, e_{2}\right)=a_{i}$ in $U(C)$ will, as usual, be denoted by $\left\langle a_{1}, \cdots\right.$, $\left.a_{n}\right\rangle$. The Witt ring of equivalence classes of $C$-spaces will be denoted by $W(C)$ and the class of a space $(E, B)$ in $W(C)$ by $[E]$. For any $C$-space $(E, B)$ there always exist $a_{1}, \cdots, a_{n}$ in $U(C)$ with $[E]=$ $\left[\left\langle a_{1}, \cdots, a_{n}\right\rangle\right],[14$, Thm. 1.16].

We shall also, very briefly, consider quadratic $C$-spaces $[19, \mathrm{pp}$. 110-111] and the left $W(C)$-module $W_{q}(C)$ of equivalence classes of quadratic $C$-spaces [19, pp. 110-111]. We shall use similar notations for quadratic spaces as for spaces.

By [14, Cor. 1.21], $R=W(C)$ is a Witt ring for the group $U(C) /(U(C))^{2}$. We shall view the signatures of $R$ as defined in $\S 1$ either as homomorphisms of $R$ to $Z$ or as homomorphisms of $U(C)$ to $\{ \pm 1\}$ sending $(U(C))^{2}$ to 1 . If $Y$ is a set of signatures of $W(C)$ (or $C$ ) we shall slightly alter one of the notations of $\S 1$ and some- 
times consider $\Gamma(Y)$ and $D([E])$ as a subset of $U(C)$ instead of $U(C) /(U(C))^{2}$.

Proposition 6.1. Let $R=W(C)$ where $C$ is a connected semilocal ring all of whose residue class fields contain at least three elements. Then $R$ is succinct.

Proof. Let $Y$ be a saturated set of signatures of $R, a_{1}, \cdots, a_{n}$ be elements of $U(C)$ and $t_{i j}, i=1, \cdots, n, j=1, \cdots, m_{i}$, elements of $\Gamma(Y)$. Denote $\perp_{i=1}^{n} \perp_{j=1}^{m_{i}}\left\langle a_{i} t_{i j}\right\rangle$ by $E$. If the element $\sum_{i=1}^{n} \sum_{j=1}^{m_{i}} a_{i} t_{i j}(U(C))^{2}$ of $Z\left[U(C) /(U(C))^{2}\right]$ is isotropic for $R$ then $[E]=\left[E^{\prime}\right]$ in $W(C)$ with rank $E^{\prime}<\operatorname{rank} E$, whence by [8, Lemma 2.2] the space $6 E$ is isotropic. But then by [8, Lemma 2.7(iii)] there exist $t_{1}, \cdots, t_{n}$ in $\Gamma(Y)$ such that $a_{1} t_{1}+\cdots+a_{n} t_{n}=0$ in $C$ and $a_{1} t_{1}+\cdots+a_{l} t_{l}$ is in $U(C)$ for all $l<n$. Thus by $\left[8\right.$, Lem. 2.10] there exist $c_{1}, \cdots, c_{n-2}$ in $U(C)$ with $\left[\left\langle a_{1} t_{1}, \cdots, a_{n} t_{n}\right\rangle\right]=\left[\left\langle c_{1}, \cdots, c_{n-2}\right\rangle\right]$ which means that the element $\sum_{1}^{n} a_{i} t_{i}(U(C))^{2}$ of $\boldsymbol{Z}\left[U(C) /(U(C))^{2}\right]$ is isotropic for $W(C)$.

CoRollary 6.2. Let $C$ be a connected semilocal ring all of whose residue class fields contain at least 3 elements. Let $Y$ denote a saturated set of signatures of $W(C)$. Then $W(C) / I(Y)$ is succinct.

Proof. This is immediate from Propositions 6.1 and 2.26.

COROLLARY 6.3. Let $C$ be a connected semilocal ring all of whose residue class fields contain at least 3 elements and such that $X(W(C)) \neq \varnothing$. Then $(W(C))_{t}=$ Nil $W(C)$ is generated as an ideal of $W(C)$ by $\left[\left\langle 1,-t^{\prime}\right\rangle\right]$ with $t^{\prime}$ in $\Gamma(X(R))$.

Proof. This is immediate from Proposition 6.1 and Corollary 2.23 .

REMARK. Corollary 6.3 was proved with the additional hypothesis " 2 in $U(C)$ " in [15, Cor. 4.19] and in full generality in [12, p. 52].

Proposition 6.4. Let $C$ be a connected semilocal ring all of whose risidue class fields contain at least three elements and let $Y$ denote a saturated set of signatures of $W(C)=R$. Then $\bar{R}=$ $W(C) I(Y)$ is strongly representational.

Proof. Let $\bar{r}_{i}, i=1,2$, be two elements of $\bar{R}$ with $\operatorname{dim}_{\bar{R}} \bar{r}_{i}=n_{i}$ and $\operatorname{dim}_{\bar{R}}\left(\bar{r}_{1}+\bar{r}_{2}\right)<n_{1}+n_{2}$. Then denote by $\sum_{j=1}^{n_{i}} a_{i j} U(C)^{2}, i=1,2$, anisotropic representatives in $Z\left[U(C) /(U(C))^{2}\right]$ of $\bar{r}_{i}$, so that 
$\sum_{j=1}^{n_{1}} a_{1 j}(U(C))^{2}+\sum_{j=1}^{n_{2}} a_{2 j}(U(C))^{2}$ is isotropic for $\bar{R}$. By [8, Thm. 2.11] there then exist $t_{i j}, i=1,2, j=1, \cdots, n_{i}$, in $\Gamma(Y)$ so that in $C$ we have $a_{11} t_{11}+a_{12} t_{12}+\cdots+a_{1 n_{1}} t_{1 n_{1}}+a_{21} t_{21}+\cdots+a_{2 n_{2}} t_{2 n_{2}}=0$ and $a_{11} t_{11}+a_{12} t_{12}+\cdots+$ $a_{1 n_{1}} t_{1 n_{1}}=u$ is a unit in $C$. Hence $-u=a_{21} t_{21}+a_{22} t_{22}+\cdots+a_{2 n_{2}} t_{2 n_{2}}$ and again by [8, Thm. 2.11], the element $u(U(C))^{2}$ lies in $D_{\bar{k}}\left(\bar{r}_{1}\right)$ and $-u(U(C))^{2}$ lies in $D_{\bar{R}}\left(\bar{r}_{2}\right)$. Therefore by Proposition 2.4, the ring $\bar{R}$ is representational. Since $\bar{R}$ is reduced, $\bar{R}$ is strongly representational by Proposition 4.2 .

Lemma 6.5. Let $C$ be a connected semilocal ring. Suppose either (a) 2 is in $U(C)$, or (b) $C$ is a local ring of characteristic 2 with maximal ideal $\mathfrak{m}$ and $\mathfrak{m}^{2}=0$. If a space $\left\langle a_{1}, \cdots, a_{n}\right\rangle$ is anisotropic then $\operatorname{dim}_{W(C)}\left[\left\langle a_{1}, \cdots, a_{n}\right\rangle\right]=n$, and for two anisotropic spaces $E_{1}$ and $E_{2}$, we have $\left[E_{1}\right]=\left[E_{2}\right]$ if and only if $E_{1} \cong E_{2}$. In addition, in case (a) and if $C$ is a field in case (b), then $\operatorname{dim}_{W^{\prime}(C)}\left[\left\langle a_{1}, \cdots, a_{n}\right\rangle\right]=n$ if and only if $\left\langle a_{1}, \cdots, a_{n}\right\rangle$ is anisotropic. In particular, if $E$ is a $C$-space with $\operatorname{dim}_{W(C)}[E]=n$, then there exists a unique anisotropic space $\left\langle a_{1}, \cdots, a_{n}\right\rangle$ with $[E]=\left[\left\langle a_{1}, \cdots a_{n}\right\rangle\right]$.

Proof. Suppose $E=\left\langle a_{1}, \cdots, a_{n}\right\rangle$ is anisotropic. If 2 is in $U(C)$ then $\operatorname{dim}_{W(C)}[E]=n$ is an immediate consequence of the definitions and Witt cancellation [9, p. 256]. If $C$ is a local ring as in case (b) and $\operatorname{dim}_{W(C)}[E]=m, m<n$, then $[E]=\left[\left\langle b_{1}, \cdots, b_{m}\right\rangle\right]$. Now repeated application of $\left[10\right.$, Satz, 3.2.1, p. 106] shows that $\left\langle b_{1}, \cdots, b_{m}\right\rangle \cong$ $M \perp L$ where $M$ is metabolic and $L$ is 0 or anisotropic with rank $L \leqq m<n$. But in the latter case $[E]=[L]$, and so by $[10$, Thm. 8.2.1, p. 119] we have $E \cong L$ which is impossible. If $L=0$, then $[E]=0$ and so by $[10$, Lem. 8.2.2, p. 119] the space $E$ is metabolic, violating the anisotropy of $E$. Thus $\operatorname{dim}_{W(C)}[E]=n$.

Suppose $E_{1}, E_{2}$ are two anisotropic spaces with $\left[E_{1}\right]=\left[E_{2}\right]$ in $W(C)$. In case (b) $E_{1} \cong E_{2}$ follows from [10, Thm. 8.2.1, p. 119] while the conclusion in case (a) is a well known consequence of Witt cancellation $[9$, p. 256].

Now suppose, in addition, in case (b) that $C$ is a field and let $E=\left\langle a_{1}, \cdots, a_{n}\right\rangle$ with $\operatorname{dim}_{W(C)}[E]=n$. If $n=0$ there is nothing to prove. By repeated applications of [10, Satz 3.2.1, p. 106] $E \cong M \perp L$ where $M$ is metabolic and $L$ is anisotropic or 0 . Now in both case (a) and (b), an anisotropic space is proper, so that if $L \neq 0$ it has an orthogonal basis $\left(\left[14\right.\right.$, Lem. 1.12]), i.e., $L \cong\left\langle c_{1}, \cdots, c_{s}\right\rangle$. By what was proved above, $\operatorname{dim}_{W(C)}[L]=s$. But $[E]=[L]$ so $L \neq 0$ if $n \neq 0$, and $s=n$. Consequently $M=0$ and $E \cong L$ is anisotropic.

Lemma 6.6. Let $C$ be a connected semilocal ring and suppose either (a) 2 is in $U(C)$ or (b) $C$ is a local ring of characteristic 2 
with maximal ideal $\mathfrak{m}$ and $\mathfrak{m}^{2}=0$. Let $E=\left\langle a_{1}, \cdots, a_{n}\right\rangle$ be an anisotropic C-space. Then for any unit $u$ in $D([E])$ there exist $x_{1}, \cdots, x_{n}$ in $C$ such that $u=a_{1} x_{1}^{2}+\cdots+a_{n} x_{n}^{2}$. In case (a), and if $C$ is a field in case (b), then this condition is also sufficient for $u$ to be in $D([E])$.

Proof. Let $u$ be a unit of $C$ which lies in $D([E])$. By Definition 1.2, there exist units $u_{2}, \cdots, u_{m}$ in $C, m \leqq n$, with $\left[\left\langle a_{1}, \cdots, a_{n}\right\rangle\right]=$ $\left[\left\langle u, u_{2}, \cdots, u_{m}\right\rangle\right]$. Now if $\left\langle u, u_{2}, \cdots, u_{m}\right\rangle$ were isotropic, then by repeated application of $\left[10\right.$, Satz 3.2 .1$, p. 106] $\left\langle u, u_{2}, \cdots, u_{m}\right\rangle \cong M \perp L$ where $M$ is metabolic, $L$ is anisotropic or 0 , and rank $L<m \leqq n$. Clearly $0 \neq[E]=\left[\left\langle u, u_{2}, \cdots, u_{m}\right\rangle\right]=[L]$, so that $L \neq 0$. Since $L$ and $E$ are both anisotropic, by Lemma 6.5 , we have $E \cong L$, a contradiction. Thus $\left\langle u, u_{2}, \cdots, u_{m}\right\rangle$ is anisotropic and by Lemma 6.5 $\left\langle u, u_{2}, \cdots, u_{m}\right\rangle \cong E=\left\langle a_{1}, \cdots, a_{n}\right\rangle$, so that $m=n$ and there exist $x_{1}, \cdots, x_{n}$ in $C$ with $u=a_{1} x_{1}^{2}+\cdots+a_{n} x_{n}^{2}$.

Now assume that there exist $x_{1}, \cdots, x_{n}$ in $C$ such that $u=$ $a_{1} x_{1}^{2}+\cdots+a_{n} x_{n}^{2}$ lies in $U(C)$. Then if $f$ is the vector $\left(x_{1}, \cdots, x_{n}\right)$ of $\left\langle a_{1}, \cdots, a_{n}\right\rangle$, the proof of Lemma 1.11 of [14] shows that $\left\langle a_{1}, \cdots a_{n}\right\rangle \cong C f \perp(C f)^{\perp}$. Since $\left\langle a_{1}, \cdots, a_{n}\right\rangle$ is anisotropic so is $(C f)^{\perp}$. If 2 is a unit in $C$ or if $C$ is a field of characteristic 2, then every anisotropic space is proper. Thus by [14, Lem. 1.12] the space $(C f)^{\perp}$ has an orthogonal basis, i.e., $(C f)^{\perp}=\left\langle u_{2}, \cdots, u_{n}\right\rangle$ for units $u_{2}, \cdots, u_{n}$ in $C$. Hence $[\langle u\rangle]+\left[\left\langle u_{2}, \cdots, u_{n}\right\rangle\right]=[E]$ and so $u$ is in $D([E])$.

Proposition 6.7. Let $C$ be a connected semilocal ring with 2 in $U(C)$. Then $R=W(C)$ is strongly representational.

Proof. Let $E_{i}=\left\langle a_{i 1}, \cdots, a_{i n_{i}}\right\rangle, i=1,2$ with $\operatorname{dim}\left[E_{i}\right]=n_{\imath}$ and suppose $\operatorname{dim}\left(\left[E_{1} \perp E_{2}\right]\right)<n_{1}+n_{2}$. Then by Lemma 6.5 , the space $E_{1} \perp E_{2}$ is isotropic so that by [2, Satz 2.7(c)] there exists a unit $u$ represented in the classical sense by $E_{1}$, such that $-u$ is represented, in the classical sense, by $E_{2}$. By Lemma 6.6 this means $u$ is in $D\left(\left[E_{1}\right]\right)$ and $-u$ is in $D\left(\left[E_{2}\right]\right)$ so that by Proposition 2.4, the ring $W(C)$ is representational.

Next, suppose for units $a, b, c$ of $C$ we have $[\langle a, b\rangle] \neq 0$ and $c$ lies in $D([\langle a, b\rangle])$. By $[8$, Rem. 1.2.4, Lem. $]$ this means $\operatorname{dim}[\langle a, b\rangle]=2$ and there exists an element $r$ in $W(C)$ with $\operatorname{dim} r \leqq 1$ so that $[\langle c\rangle]+r=[\langle a, b\rangle]$. Again by [8, Rem. 1.24, Lem.] the case $\operatorname{dim} r=0$ is impossible, so that there is unit $d$ in $C$ with $[\langle c, d\rangle]=[\langle a, b\rangle]$. By Lemma 6.5 this means $\langle c, d\rangle \cong\langle a, b\rangle$. If $\{e, f\}$ is the canonical basis for $\langle a, b\rangle$, there exist elements $\alpha, \beta$ in $C$ with $B(\alpha e+\beta f$, $\alpha e+\beta f)=c$, i.e., $\alpha^{2} a+\beta^{2} b=c$. But then $\{\alpha e+\beta f, \beta b e-\alpha a f\}$ is 
easily seen to be another orthogonal basis of $\langle a, b\rangle$, so that $\langle a, b\rangle \cong$ $\langle c, c a b\rangle$, and $W(C)$ is strongly representational.

We shall now see that if 2 is not a unit in $C$, the ring $W(C)$ need not be representational.

EXAMPLE 6.8. Let $F$ be a field of characteristic 2 with $\left[F: F^{2}\right]$ infinite. For example, $F=F_{0}\left(x_{1}, \cdots\right)$ where $F_{0}$ is a field of characteristic 2 and $x_{1}, \cdots$ are countably infinitely many algebraically independent elements over $F_{0}$. Let

$$
\left\{1, a_{2}, a_{3}, \cdots, b_{1}, b_{2}, \cdots\right\}
$$

be a basis of $F$ over $F^{2}$. Let $C$ be the ring of dual numbers over $F$, i.e., $C=F+F x$ with $x^{2}=0$. Then $C$ is local with maximal ideal $\mathfrak{m}=F x$ and $C / \mathfrak{m} \mathfrak{\imath} \cong F$. Note that $C^{2}=F^{2}$.

Consider next the $C$-spaces $E_{1}=\left\langle 1, a_{2}, \cdots, a_{n}\right\rangle, E_{2}=\left\langle b_{1}, b_{1}+x\right.$, $\left.b_{3}, \cdots, b_{m}\right\rangle$. We first verify that $E_{1} \perp E_{2}$ is anisotropic: If $c_{1}^{2}+$ $\sum_{2}^{n} c_{i}^{2} a_{\imath}+c_{1}^{\prime 2} b_{1}+c_{2}^{\prime 2}\left(b_{1}+x\right)+\sum_{3}^{m} c_{i}^{\prime 2} b_{i}=0$, then since $C^{2}=F^{2}$ we have $c_{\imath}^{2}=0, c_{2}^{\prime 2}=0$ and $c_{i}^{\prime 2}=0, i \neq 2$. Hence all $c_{i}$ and $c_{i}^{\prime}$ lie in $\mathfrak{m}$ and $E_{1} \perp E_{2}$ is anisotropic. If $R=W(C)$, Lemma 6.5 shows $\operatorname{dim}_{Z}\left(\left[E_{1} \perp E_{2}\right]\right)=$ $n+m=\operatorname{dim}_{R}\left(\left[E_{1}\right]\right)+\operatorname{dim}_{R}\left(\left[E_{2}\right]\right)$.

Let $\left\{v_{i}\right\}_{2=1, \ldots, n},\left\{w_{i}\right\}_{2=1, \ldots, m}$ be the canonical bases of $E_{1}$ and $E_{2}$ respectively. Then in $E_{1} \perp E_{2}$, we have $B\left(v_{1}+w_{1}+w_{2}, v_{1}+w_{1}+w_{2}\right)=$ $1+b_{1}+b_{1}+x=1+x$ and by the first part of the proof of Lemma 1.11 of [14], $E_{1} \perp E_{2}=C\left(v_{1}+w_{1}+w_{2}\right) \perp\left(C\left(v_{1}+w_{1}+w_{2}\right)\right)^{\perp}$. Clearly $v_{2}$ is in $\left(C\left(v_{1}+w_{1}+w_{2}\right)\right)^{\perp}$ and $B\left(v_{2}, v_{2}\right)=a_{2}$ in $U(C)$. Therefore by [14, Lem. 1.12] $\left(C\left(v_{1}+w_{1}+w_{2}\right)\right)^{\llcorner}=\left\langle e_{2}, \cdots, e_{m+n}\right\rangle$ with $e_{i}$ in $U(C)$. Thus $E_{1} \perp E_{2}=\left\langle 1+x, e_{2}, \cdots, e_{m+n}\right\rangle$ and according to Definition 1.2, we have $1+x$ lies in $D\left(\left[E_{1} \perp E_{2}\right]\right)$.

Let $g_{i}$ be units of $C$ in $D\left(\left[E_{i}\right]\right), i=1,2$. By Lemma 6.6 this yields the existence of elements $\varphi_{1}, \cdots, \varphi_{n}, \lambda_{1}, \cdots, \lambda_{m}$ in $C$ with

$$
g_{1}=\varphi_{1}^{2}+\sum_{3}^{n} a_{i} \varphi_{i}^{2} \neq 0, g_{2}=b_{1}\left(\lambda_{1}^{2}+\lambda_{2}^{2}\right)+\sum_{i}^{m} b_{i} \lambda_{i}^{2}+\lambda_{2}^{2} x
$$

with

$$
h=b_{1}\left(\lambda_{1}^{2}+\lambda_{2}^{2}\right)+\sum_{3}^{m} b_{i} \lambda_{i}^{2} \neq 0 .
$$

The space $\left\langle g_{1}, g_{2}\right\rangle$ is again anisotropic: For if for $y_{1}, y_{2}$ in $C$ $g_{1} y_{1}+g_{2} y_{2}=0$ then $\lambda_{2}^{2} y_{2}^{2}=0$. If $y_{2}^{2}=0$ then $g_{1} y_{2}^{2}=0$ implies $y_{1}^{2}=0$ so $y_{1}, y_{2}$ lie in $n t$. If $y_{2}^{2} \neq 0$, then $\lambda_{2}^{2}=0$ and the independence of $1, a_{2}, \cdots, b_{1}, b_{3}, \cdots$ over $F^{2}=C^{2}$ forces $y_{1}^{2}=0, y_{2}$ in $\mathrm{n}$ and $g^{2}=0$, which is impossible.

Suppose now that $1+x$ were in $D\left(\left[\left\langle g_{1}, g_{2}\right\rangle\right]\right)$. Again Lemma 6.6 would yield elements $\alpha, \beta$ in $C$ with 


$$
1+x=\alpha^{2} g_{1}+\beta^{2} h+\beta^{2} \lambda_{2}^{2} x .
$$

Then $\beta^{2} \lambda_{2}^{2}=1$ and $\alpha^{2} g_{1}+\beta^{2} h=1$. The independence of $1, a_{2}, \cdots$, $b_{1}, \cdots$ over $F^{2}=C^{2}$ then forces $\beta^{2} h=0$, which since $\beta^{2} \neq 0$, forces $h=0$, a contradiction. Thus $1+x$ does not lie in $D\left(\left[\left\langle g_{1}, g_{2}\right\rangle\right]\right)$ for any $g_{i}$ in $D\left(\left[E_{i}\right]\right), i=1,2$, and $R=W(C)$ is not representational.

REMARKS 6.9(i). The inclusion relations \{Witt rings for $G$ \} $\supset$ \{succinct Witt rings for $G\} \supset\{$ representational Witt rings for $G$ \} are all proper: In [8, Remark 1.19] an example of a torsion free Witt ring $R$ for $G$ which is not dimensional was given. From Proposition 2.20 with $Y=X(R)$ it then follows that $R$ is not succinct. Furthermore if $C$ is the local ring of Example 6.8, the Witt ring $W(C)$ is succinct by Proposition 6.1 but fails to be representational.

(ii) The local ring $C$ of Example 6.8 also furnishes another example of a 2-fold bilinear Pfister form which is isotropic but whose class in $W(C)$ is $\neq 0$ : Let $P_{0}=\langle 1,1+x\rangle\left\langle 1,1+a_{2} x\right\rangle=$ $\left\langle 1,1+x, 1+a_{2} x, 1+\left(a_{2}+1\right) x\right\rangle$. If $(\alpha, \beta, \gamma, \delta)$ is an isotropic element of $P_{0}$ then $\alpha^{2}+\beta^{2}(1+x)+\gamma^{2}\left(1+a_{2} x\right)+\delta^{2}\left(1+\left(a_{2}+1\right) x\right)=0 . \quad \mathrm{A}$ routine computation shows that this forces $\alpha^{2}=\beta^{2}=\gamma^{2}=\delta^{2}$, and, indeed the element $(1,1,1,1)$ is a primitive isotropic element of $P_{0}$. Hence if $V \subset P_{0}$ is a totally isotropic submodule of $P_{0}$, it is easily seen that $[V / \mathfrak{m} V: F]=1$. But if $P_{0}$ is metabolic, then $P_{0}$ contains a direct summand $W$ which is totally isotropic and of rank $2[10$, Satz 3.2.1, p. 106] and necessarily $[W / \mathfrak{m} W: F]=2$, hence $P_{0}$ is not metabolic and therefore by $\left[10\right.$, Lem. 8.2.2, p. 119], $\left[P_{0}\right] \neq 0$ in $W(C)$. In [1, Bemerkung (2.3), pp. 146-147] another such example is given with $C=\boldsymbol{Z} / 4 \boldsymbol{Z}$. Our example shows that this pathological behavior of isotropic bilinear Pfister forms can also occur for "very large" residue class fields of characteristic 2 .

Proposition 6.10. If $C$ is a field of characteristic 2 then $W(C)$ is strongly representational.

Proof. Let $r_{i}$ be elements of $R=W(C)$ with $\operatorname{dim}_{W^{(C)}} \boldsymbol{r}_{i}=n_{i}$, $i=1,2$ and $\operatorname{dim}_{W(C)}\left(r_{1}+r_{2}\right)=n_{1}+n_{2}$. By Lemma 6.5 we have $r_{1}=$ $\left[\left\langle a_{1}, \cdots, a_{n_{1}}\right\rangle\right], r_{2}=\left[\left\langle b_{1}, \cdots, b_{n_{2}}\right\rangle\right]$ with $\left\langle a_{1}, \cdots, a_{n_{1}}\right\rangle$ and $\left\langle b_{1}, \cdots, b_{n_{2}}\right\rangle$ anisotropic and $\left\langle a_{1}, \cdots, a_{n_{1}}, b_{1}, \cdots, b_{n_{2}}\right\rangle$ also anisotropic. Now let $c$ lie in $D\left(r_{1}+r_{2}\right)$. By Lemma 6.6, there exist $x_{1}, \cdots, x_{n_{1}}, y_{1}, \cdots, y_{n_{2}}$ in $C$ with $c=\sum_{1}^{n_{1}} a_{i} x_{i}^{2}+\sum_{1}^{n_{2}} b_{i} y_{i}^{2} \neq 0$. If both $c_{1}=\sum_{1}^{n_{1}} a_{i} x_{i}^{2}$ and $c_{2}=$ $\sum_{1}^{n_{2}} b_{i} y_{i}^{2}$ are $\neq 0$, then again by Lemma $6.6, c_{i}$ lies in $D\left(r_{i}\right), i=1,2$ and since $\left\langle c_{1}, c_{2}\right\rangle$ is clearly anisotropic, by Lemma $6.6 c$ is in $D\left(\left[\left\langle c_{1}, c_{2}\right\rangle\right]\right)$. If $c_{2}=0$, say, then once more by Lemma 6.6 we have $c$ lies in $D\left(\left[\left\langle c_{1}, b_{1}\right\rangle\right]\right)$ with $c_{1}$ in $D\left(r_{1}\right)$ and $b_{1}$ in $D\left(r_{2}\right)$ so that $R$ is representational. 
Now let $[\langle a, b\rangle] \neq 0$ and $c$ be in $D([\langle a, b\rangle])$. By [8, Rem. 1.24, Lem.], $\operatorname{dim}_{W(C)}[\langle a, b\rangle]=2$ so that $\langle a, b\rangle$ is anisotropic by Lemma 6.5. Just as in the proof of Proposition 6.7 there exists $d$ in $U(C)$ with $[\langle c, d\rangle]=[\langle a, b\rangle]$. By Lemma 6.5 this implies $\langle c, d\rangle \cong\langle a, b\rangle$ whence, just as in the proof of Proposition 6.7, we have $\langle a, b\rangle \cong\langle c, c a b\rangle$ and $W(C)$ is strongly representational.

Corollary 6.11. Let $C$ be (a) either a connected semilocal ring with 2 in $U(C)$ or a field of characteristic 2, or (b) a connected semilocal ring with all residue class fields containing at least 3 elements. Then if $r$ is a round element of $R=W(C)$ in case (a) and of $R=W(C) / I(Y)$, for a saturated set of signatures $Y$ in case (b), then $\operatorname{Ann}_{R}(r)=\mathfrak{a}(D(r))$.

Proof. In case (a), Proposition 6.7 and Proposition 6.10 and in case (b), Proposition 6.4 shows that the $R$ in question is representational. Theorem 2.15 then completes the proof.

[8, Thm. 2.11] in case (b) and Lemma 6.6 in case (a) yield an explicit description of $D(r)$. Thus by Lemma 6.6, the case (a) in Corollary 6.11 is already contained in [11, Thm. 4.1 and Bemerkung 4.4]. We now show how Lemma 2.13 combines with [2, Satz 2.7] to reprove [11, Thm. 4.1].

Let $(E, q)$ be a quadratic $C$-space. A unit $u$ of $C$ is said to be represented by $(E, q)$ if there is a primitive element $e$ in $E$ with $q(e)=u$. As in Definition 2.14, the space $(E, q)$ is called round if $[u q]=[q]$ in $W_{q}(C)$ for all units represented by $q$.

Proposition 6.12. Let $C$ be a connected semilocal ring all of whose residue class fields contain at least three elements and $(E, q)$ a round quadratic $C$-space of rank $\geqq 2$. Then if $R=W(C)$ and $[q] \neq 0$ in $W_{q}(C)$, we have $\operatorname{Ann}_{R}([q])=a(T)$ where $T$ is the set of units represented by $[q]$.

Proof. Let $r=\left[\left\langle a_{1}, \cdots, a_{n}\right\rangle\right]$ lie in $\operatorname{Ann}_{R}([q])$. Then $\left\langle a_{1}, \cdots, a_{n}\right\rangle \otimes E$ is hyperbolic and so, in particular, isotropic. Now $\left\langle a_{1}, \cdots, a_{n}\right\rangle \otimes E=$ $\perp_{1}^{n} a_{2} E$. If $n=1$, then $r$ is a unit in $W(C)=R$ and so $[q]=0$. If $n=2$ then by [2, Satz 2.7(b)] there are units $u_{1}, u_{2}$ in $C$, represented by $\left(E, a_{1} q\right)$ and $\left(E, a_{2} q\right)$ respectively, with $u_{1}+u_{2}=0$. Now $u_{\imath}=a_{i} q\left(e_{i}\right)=a_{i} t_{i}$ with $t_{i}$ in $T, i=1,2$. Hence $a_{1} t_{1}+a_{2} t_{2}=0$ and $\left[\left\langle t_{i}\right\rangle\right][q]=[q]$. Then by $\left[10\right.$, Satz 3.2.1, p. 106] we have $\left[\left\langle a_{1} t_{1}, a_{2} t_{2}\right\rangle\right]=0$ so the element $a_{1} t_{1}(U(C))^{2}+a_{2} t_{2}(U(C))^{2}$ of $Z\left[U(C) /(U(C))^{2}\right]$ is isotropic for $R$. If $n>2$ we apply [2, Satz 2.7(b)] to $\perp_{1}^{n-2} a_{i} E \perp a_{n-1} E \perp a_{n} E$ to obtain units $v, v_{n-1}, v_{n}$ in $C$, represented by $\perp_{1}^{n-2} a_{\imath} E, a_{n-1} E, a_{n} E$ respectively, such that $v+v_{n-1}+v_{n}=0$. As before, $v_{n-1}=a_{n-1} \widetilde{t}_{n-1}$, 
$v_{n}=a_{n} \widetilde{t}_{n}$ with $\widetilde{t}_{n-1}, \widetilde{t}_{n}$ in $T$. A final application of [2, Satz 2.7(b)] to the unit $v$ yields units $\tilde{t}_{1}, \tilde{t}_{2}, \cdots, \tilde{t}_{n-2}$ represented by $q$ such that $v=a_{1} \widetilde{t}_{1}+\cdots+a_{n-2} \widetilde{t}_{n-2}$, so that we finally have $a_{1} \widetilde{t}_{1}+\cdots+a_{n} \widetilde{t}_{n}=0$ with $a_{1} \widetilde{t}_{1}+\cdots+a_{n-2} \widetilde{t}_{n-2}$ in $U(C)$. By $[8$, Lem. 2.10] the element $\sum_{1}^{n} a_{\imath} \tilde{t}_{i}(U(C))^{2}$ of $Z\left[U(C) /(U(C))^{2}\right]$ is isotropic for $R$. Hence by Lemma 2.13, we have $\operatorname{Ann}_{R}([E])=\mathfrak{a}\left(T^{\prime}\right)$ where $T^{\prime}$ is the subgroup of $U(C)$ consisting of all units $t$ with $[\langle t\rangle][q]=[q]$. That $T=T^{\prime}$ is standard [1, Bemerkung (1.2), p. 127].

REMARK 6.13. As we pointed out in $\S 4$, most of the proofs in that section are adaptations to our situation of the corresponding proofs in $[6,7,16]$. There the results are proved for spaces over a field of characteristic not two and use the relations of isometry and $p$-chain equivalence where we use equality in a Witt ring for $G$.

In Propositions 6.7 and 6.10 we showed that $W(C)$ is strongly representational when $C$ is a connected semilocal ring with 2 in $U(C)$ or any field. A little argument now shows that the original Elman-Lam results are a consequence of ours up to isometry (rather than $p$-chain equivalence) for $C$ a connected semilocal ring with 2 in $U(C)$ and even remain largely true for $C$ a field of characteristic 2: Let $P, Q$ be two $n$-fold Pfister spaces over $C$, where $C$ is as above. If $P$ is isotropic then by Lemma $6.5 \operatorname{dim}[P]<2^{n}$ so that by Corollary 4.5, we have $[P]=0$. If $C$ is a field of characteristic 2, then $P$ is metabolic by [10, Lem. 8.2.2, p. 119]; if $C$ is a connected semilocal ring with 2 in $U(C)$, then using Witt cancellation [9, p. 256] we see that $P$ is hyperbolic. Thus Corollary 4.5 yields [6, Cor. 2.3] exactly when $C$ is a connected semilocal ring with 2 in $U(C)$ and a modified version, substituting "metabolic" for hyperbolic when $C$ is a field of characteristic 2. In either case, if $[P]=[Q] \neq 0$ then by Corollary 4.5 and Lemma 6.5 , the spaces $P$ and $Q$ are anisotropic and hence isometric. If $[P]=[Q]=0$ then we can, of course, only conclude that $P$ and $Q$ are hyperbolic and so $P \cong Q$, in case $C$ is a connected semilocal ring with 2 in $U(C)$.

These observations together with Lemmas 6.5 and 6.6 are sufficient to allow deduction of [7, Thm. $2.1(1,2,3)]$ and Theorem 2.7 and Remark, Corollaries 1.9, 1.10, 2.5 and part of Corollary 2.4 of [6] from their analogues in Propositions 4.9, 4.13, Corollaries 4.6, 4.10, 4.11, 4.12 and Lemmas 4.3 and 4.7 for spaces over connected semilocal rings with 2 in $U(C)$, or for fields of characteristic 2 whenever the statements in $[6,7]$ are restricted to anisotropic spaces. Furthermore, these observations also yield, up to isometry, [6, Prop. 2.2, Thm. 2.6] from Propositions 4.4 and 4.8, respectively, when, in the notations of [6, Prop. 2.2, Thm. 2.6], the Pfister spaces, $\varphi$ and $\tau \gamma$ are anisotropic. If $\phi$ and $\tau \gamma$ are isotropic, or as 
we have noted, $[\varphi]=[\tau \gamma]=0$, then in the case of a connected semilocal ring $C$ with 2 in $U(C)$, it is an easy matter to see that [6, Prop. 2.2 and Thm. 2.6] follow in this case also. Finally, [6, Prop. 4.4, Thm. 4.5] follow readily in both cases for anisotropic Pfister spaces from Propositions 4.15 and 4.16, respectively, noting that for an arbitrary $C$-space $E$, we have $\operatorname{rank} E=\operatorname{dim}[E]+2 \times$ (Witt index of $E$ ). Again it is an easy matter, in the semilocal case, to deduce [6, Prop. 4.4, Thm. 4.5] whenever, in the notation of [6, Prop. 4.4, Thm. 4.5] the Pfister spaces $\varphi$ or $\gamma$ are isotropic.

REMARK 6.14. We owe the substance of this remark to a conversation with Roger Ware.

Let $R$ be a Witt ring for $G$ and also for $H$. Then it may well happen that for $r$ in $R$ both $\operatorname{dim} r$ and the image of $D(r)$ in $R$ depend on the particular presentation of $R$. For example, if $R=R_{t}$ and $G=U(R)$ with $(U(R))^{2}=1$, then $R$ is a Witt ring for $U(R)$ and if $u_{i}$ are elements of $G$, then $\sum_{1}^{2 n} u_{i}$ lies in the unique maximal ideal of $R$ containing 2 [14, Lem. 2.13] and thus is nilpotent [14, Prop. 3.16]. Thus for $u_{i}$ in $G$ the element $\sum_{1}^{2 n+1} u_{i}=r$ is again in $G$ and so $\operatorname{dim} r=1$. Moreover for $u_{i}$ in $G$ we have $\sum_{1}^{2 n+2} u_{i}=u_{1}+$ $\sum_{2}^{2 n+2} u_{i}$ so that $\operatorname{dim}\left(\sum_{1}^{2 n+2} u_{i}\right)=2$ or 0 . Furthermore, if $u$ is in $G$ we have $D(u)=u$ and for $u_{1}, u_{2}$ in $G, u_{1} \neq-u_{2}, D\left(u_{1}+u_{2}\right)=G$ because for any $v$ in $G$ we have $u_{1}+u_{2}=v+\left(u_{1}+u_{2}-v\right)$. Using Proposition 2.4 it is then easily verified that $R$ is representational as a Witt ring for $U(R)$. Now let $F$ be a field of characteristic not two, containing $\sqrt{-1}$, and possessing an anisotropic space of rank $3, \boldsymbol{Q}(\mathrm{i})$ is such a field, for example. Then since $R=W(F)$ has characteristic 2 it is easily verified that $(U(R))^{2}=1$ and $R$ as a Witt ring for $U(F) /(U(F))^{2}$ has elements of dimension 3 by Lemma 6.5 , whereas as a Witt ring for $U(R)$ it has only elements of dimension 1 and 2.

Next, let $R=Z / 8 Z$ and treat $R$ first as a Witt ring for $G=\{1\}$. Then $\operatorname{dim} \overline{3}=3$ since otherwise by $[8$, Rem. 1.24, Lem. $] \operatorname{dim} \overline{3}=1$ and $\overline{3}= \pm \overline{1}$ is impossible. Also $D(\overline{3})=\{1\}$ is immediately verified. Furthermore, $\overline{3}+\overline{3}=-\overline{1}+-\overline{1}$ so $\operatorname{dim}(\overline{3}+\overline{3})=2$. By Proposition 2.4 then, since $D(\overline{3})=\{1\}$, the Witt ring $Z / 8 Z$ is not representational as a Witt ring for $G=\{1\}$.

On the other hand, $U(\boldsymbol{Z} / 8 \boldsymbol{Z})=\{\overline{1}, \overline{3}, \overline{5}, \overline{7}\}$ so that $(U(\boldsymbol{Z} / 8 \boldsymbol{Z}))^{2}=1$. Thus by the above, $\boldsymbol{Z} / 8 \boldsymbol{Z}$ is a representational Witt ring for $U(\boldsymbol{Z} / 8 \boldsymbol{Z})$ with $\operatorname{dim}(\overline{3})=1$. The Witt ring $\boldsymbol{Z} / 8 \boldsymbol{Z}$ for $U(\boldsymbol{Z} / 8 \boldsymbol{Z})$ is, however not strongly representational since $\overline{1}+\overline{1}=\overline{3}+\overline{7}$ so that $\overline{3}$ lies in $D(\overline{1}+\overline{1})$ but $\overline{1}+\overline{1} \neq \overline{3}+\overline{3}(\overline{1} \cdot \overline{1})$. Thus by Propositions 6.7 and $6.10, Z / 8 Z$ cannot be isomorphic to $W(C)$ for $C$ a semilocal ring 
with 2 in $U(C)$ or a field of characteristic 2 as either a Witt ring for $\{1\}$ or for $U(Z / 8 Z)$.

The situation is quite different if $R_{t}=$ Nil $R=0$, for then $U(R)=\bar{G}^{\prime}[14$, Rem. 3.22, Thm. 3.23], where $R$ is a Witt ring for $G$. Hence the dimension in $R$ is independent of $G$ and if $R$ is also a Witt ring for a group $H$ of exponent 2 then $D_{G}(r)$ and $D_{H}(r)$, with the obvious notation, have the same image in $R$.

7. Remarks on [8] and [20]. Some of the results of this paper are applicable to $R=W(C) / I(Y)$, using the notations of $\S 6$, to obtain alternate proofs of some of the results of [8] and [20]. Proposition 6.1 shows that $W(C)$ is succinct for a connected semilocal ring $C$ all of whose residue class fields contain at least 3 elements. Consequently, [8, Cor. 2.12] becomes a direct consequence of Proposition 2.20, while Proposition 2.22 yields a proof of [8, Lem. 2.14] which does not depend on [11, Thm. 4.1]. Theorems 3.3 and 3.6 are generalizations of [8, Thm. 3.2 (i), (ii) and Cor. 3.12] respectively. In order to deduce these results of [8] from Theorems 3.3 and 3.6 we require $[8, \mathrm{Thm} .2 .11]$ and

Lemma 7.1. Let $R=W(C)$ with $C$ a connected semilocal ring all of whose residue class fields contain at least 3 elements, and $Y a$ saturated set of signatures of $R$. Let $a_{i}, i=1, \cdots, n$, denote units of $C$. Then there exist elements $t_{i}$ of $\Gamma(Y)$ such that $\sum_{1}^{n} a_{\imath} t_{\imath}(U(C))^{2}$ in $Z\left[U(C) /(U(C))^{2}\right]$ is isotropic for $R$ in the sense of Definition 1.3, if and only if there exist $s_{i}$ in $\Gamma(Y)$ such that $a_{1} s_{1}+\cdots+a_{n} s_{n}=0$ in $C$ and $a_{1} s_{1}+\cdots+a_{l} s_{l}$ is in $U(C)$ for all $l<n$.

Proof. By [8, Thm. 2.11], the sum $a_{1} s_{1}+\cdots+a_{n} s_{n}=0$ in $C$, with $a_{1} s_{1}+\cdots+a_{l} s_{l}$ a unit for all $l<n$, if and only if $z=\sum_{1}^{n} a_{i}(U(C))^{2}$ in $Z\left[U(C) /(U(C))^{2}\right]$ is isotropic for $\bar{R}=W(C) / I(Y)$. By Proposition 6.1, $R$ is succinct, so that Lemma 3.1 shows that $z$ is isotropic for $\bar{R}$ if and only if there exist $t_{i}$ in $\Gamma(Y)$ such that $\sum_{1}^{n} a_{i} t_{i}(U(C))^{2}$ is isotropic for $R$, in the sense of Definition 1.3.

Using Propositions 6.4 and 2.24, we can apply Theorem 3.9 to $R=W(C) / I(Y)$ where the notation is as defined in Lemma 7.1. This application includes the special case proved as part of [4, Satz 24 and 25] of the equivalence of WAP and SAP in case $C$ is a field of characteristic $\neq 2$. By virtue of Proposition 6.4, Theorem 3.9 can also be applied to $R=W(C)$. This yields [20, Thm. 2.2] without needing the assumption that 2 be in $U(C)$. It does not, however, cover all of [13, Thm. 1], where the equivalence of WAP and SAP is proved for arbitrary connected semilocal rings and for the Witt ring of hermitian forms over an arbitrary connected semilocal ring 
with involution.

\section{References}

1. R. Baeza, Quadratische Formen über semilokalen Ringen, Habilitationschrift, Saarbrücken (1975).

2. R. Baeza and M. Knebusch, Annulatoren von Pfisterformen über semilokalen Ringen, Math. Z., 140 (1974), 41-62.

3. E. Becker and L. Bröcker, On the description of the reduced Witt ring, J. Algebra, 52 (1978), 328-346.

4. E. Becker and E. Köpping, Reduzierte quadratische Formen und Semiordnungen reeler Körper, Abh. Math. Sem. Hamburg, 46 (1977), 143-177.

5. T. C. Craven, The Boolean space of orderings of a field, Trans. Amer. Math. Soc., 209 (1975), 225-235.

6. R. Elman and T. Y. Lam, Pfister Forms and K-theory of fields, J. Algebra, 23 (1972), 181-213.

7. - Quadratic forms over formally real fields and Pythagorean fields, Amer. J. Math., 94 (1972), 1155-1194.

8. J. L. Kleinstein and A. Rosenberg, Signatures and semisignatures of abstract Witt rings and Witt rings of semilocal rings, Canad. J. Math., 30 (1978), 872-895.

9. M. Knebusch, Isometrien über semilokalen Ringen, Math. Z., 108 (1969), 255-268. 10. - Grothendieck-und Wittringe von nichtausgearteten symmetrischen Bilinearformen, Sitzber. Heidelberg Akad. Wiss., (1969/70), 93-157.

11. - Runde Formen über semilokalen Ringen, Math. Ann., 193 (1971), 21-34.

12. - Generalization of a theorem of Artin-Pfister, J. Algebra, 36 (1975), $46-67$.

13. - Remarks on the paper "Equivalent topological properties of the space of signatures of a semilocal ring" by $A$. Rosenberg and $R$. Ware, Pub. Math., 24 (1977), 181-188.

14. M. Knebusch, A. Rosenberg and R. Ware, Structure of Witt rings and quotients of abelian group rings, Amer. J. Math., 94 (1972), 119-155.

15. — Signatures on semilocal rings, J. Algebra. 26 (1973), 208-250.

16. T. Y. Lam, The algebraic theory of quadratic forms, W. A. Benjamin, Reading, Mass., 1973.

17. M. Marshall, Classification of finite spaces of orderings, Canad. J. Math., 31 (1979), 320-330.

18. - Quotients and inverse limits of spaces of orderings, Canad. J. Math., to appear.

19. J. Milnor and D. Husemoller, Symmetric Bilinear Forms, Ergeb. d. Math. 73, 1973, Springer Verlag, New York-Heidelberg-Berlin.

20. A. Rosenberg and R. Ware, Equivalent topological properties of the space of signatures of a semilocal ring, Pub. Math., 23 (1976), 283-289.

21. B. L. van der Waerden, Moderne Algebra, vol. 2, 2nd rev. ed., J. Springer, Berlin, 1940.

Received March 21, 1978. Partially supported by NSF Grant MCS73-04876.

SUNY AT STONY BRook

STONY BROOK, NY 11794

AND

CORNELL UNIVERSITY

ITHACA, NY 14853 



\section{PACIFIC JOURNAL OF MATHEMATICS}

\section{EDITORS}

DONALD BABBITT (Managing Editor)

University of Galifornia

Los Angeles, California 90024

HUGo RossI

University of Utah

Salt Lake City, UT 84112

C. C. MOORE AND ANDREW OGG

University of California

Berkeley, CA 94720
J. DUGUNDJI

Department of Mathematics University of Southern California Los Angeles, California 90007

R. FINN AND J. MILGRAM Stanford University Stanford, California 94305

\section{ASSOCIATE EDITORS}

E. F. BECKENBACH

B. H. NEUMANN

F. WOLF

K. YosHIDA

\section{SUPPORTING INSTITUTIONS}

UNIVERSITY OF BRITISH COLUMBIA CALIFORNIA INSTITUTE OF TECHNOLOGY UNIVERSITY OF CALIFORNIA MONTANA STATE UNIVERSITY UNIVERSITY OF NEVADA, RENO NEW MEXICO STATE UNIVERSITY OREGON STATE UNIVERSITY UNIVERSITY OF OREGON
UNIVERSITY OF SOUTHERN CALIFONIA STANFORD UNIVERSITY UNIVERSITY OF HAWAII UNIVERSITY OF TOKYO UNIVERSITY OF UTAH WASHINGTON STATE UNIVERSITY UNIVERSITY OF WASHINGTON 


\section{Pacific Journal of Mathematics}

\section{Vol. 86, No. $1 \quad$ November, 1980}

Gert Einar Torsten Almkvist, Invariants, mostly old ones .............. 1

Hyman Bass, Groups of integral representation type ................ 15

A. Białynicki-Birula, On action of SL(2) on complete algebraic

varieties........................................

Frederick Paul Greenleaf and Martin Allen Moskowitz, Groups of

automorphisms of Lie groups: density properties, bounded orbits, and

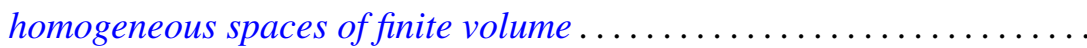

Raymond Taylor Hoobler, A cohomological interpretation of Brauer groups

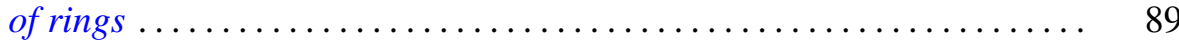

Irving Kaplansky, Superalgebras ........................ 93

Jerrold Lewis Kleinstein and Alex I. Rosenberg, Succinct and

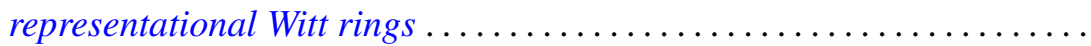

E. R. Kolchin, On universal extensions of differential fields ............ 139

Andy R. Magid, Analytic subgroups of affine algebraic groups. II ....... 145

Calvin Cooper Moore, The Mautner phenomenon for general unitary

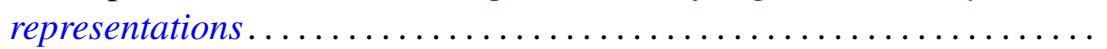

George Daniel Mostow, On a remarkable class of polyhedra in complex hyperbolic space ................................ 171

Brian Lee Peterson, Extensions of pro-affine algebraic groups. II . . . . . . 277

John Henry Reinoehl, Lie algebras and affine algebraic groups......... 287

Maxwell Alexander Rosenlicht, Differential valuations .

John Brendan Sullivan, The second Lie algebra cohomology group and Weyl modules..................................... 321

Moss Eisenberg Sweedler, Right derivations and right differential

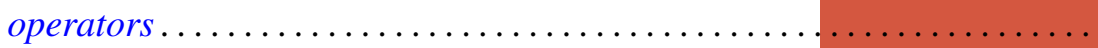

Bostwick Frampton Wyman, Time varying linear discrete-time systems. II. Duality.................................. 